
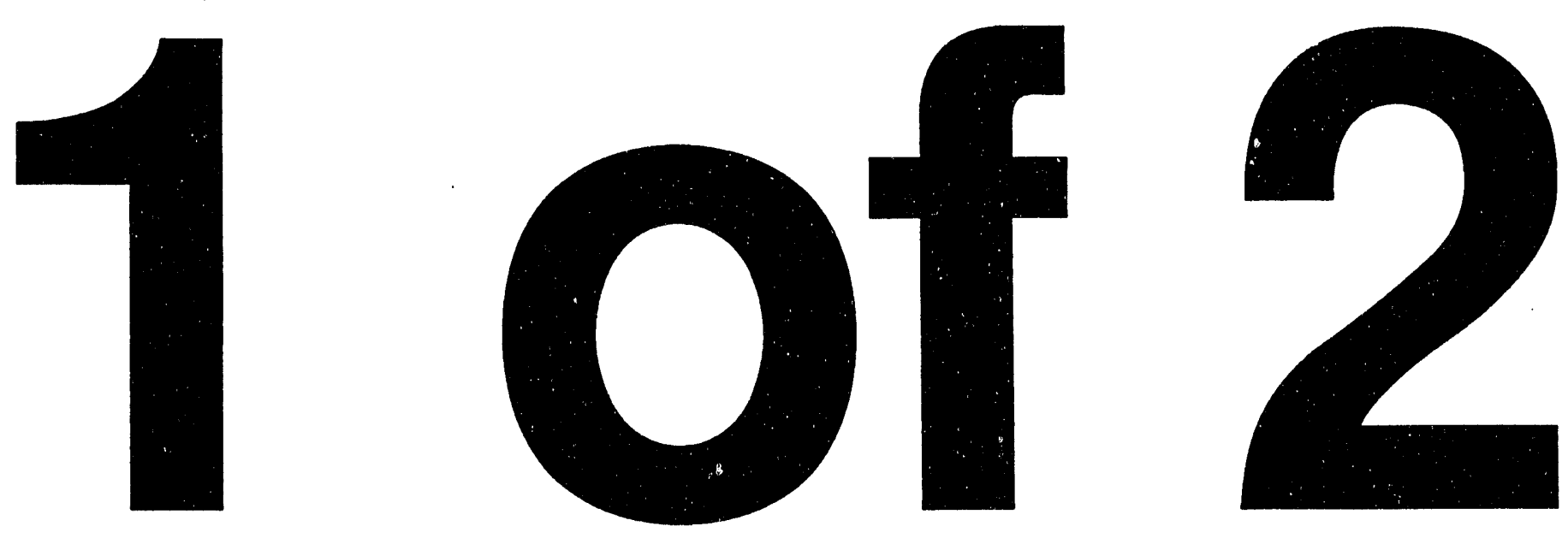
SAND93-0737

Distribution

Unlimited Release

Printed August 1994

Category UC-722

\title{
MELTER: A Model of the Thermal Response of Cargos Transported in the Safe-Secure Trailer Subject to Fire Environments for Risk Assessment Applications
}

\author{
M. E. Larsen \\ Transportation Analysis Department 9614 \\ Sandia National Laboratories \\ Albuquerque, NM 87185
}

\begin{abstract}
MELTER is an analysis of cargo responses inside a fire-threatened Safe-Secure Trailer (SST) developed for the Defense Program Transportation Risk Assessment (DPTRA). Many simplifying assumptions are required to make the subject problem tractable. MELTER incorporates modeling which balances the competing requirements of execution speed, generality, completeness of essential physics, and robustness. Input parameters affecting the analysis include those defining the fire scenario, those defining the cargo loaded in the SST, and those defining properties of the SST. For a specified fire, SST, and cargo geometry MELTER predicts the critical fire duration that will lead to a failure. The principal features of the analysis include: a) Geometric considerations to interpret fire-scenario descriptors in terms of a thermal radiation boundary condition, b) a simple model of the SST's wall combining the diffusion model for radiation through optically-thick media with an endothermic reaction front to describe the charring of rigid foam in the SST wall, c) a transient radiation enclosure model, d) a one-dimensional, spherical idealization of the shipped cargos providing modularity so that cargos of interest can be inserted into the model, and e) associated numerical methods to integrate coupled, differential equations and find roots.
\end{abstract}




\section{Contents}

1 Introduction 1

2 MELTER Theory $\quad 3$

2.1 Introduction . . . . . . . . . . . . . . . . . 3

2.2 Fire Boundary Condition . . . . . . . . . . . . . . . 5

2.2.1 Fire Geometry .................. 5

2.2 .2 Inferrid Parameters . . . . . . . . . . . . . . 6

2.2.3 View Factors and Exposed Area . . . . . . . . . . . . 8

2.3 Charring Foam Wall Model . . . . . . . . . . . . . . . . 12

2.3.1 Wall Model ... . . . . . . . . . . . . . . 12

2.3.2 The Charring Floor Model . . . . . . . . . . . . . 15

2.4 Enclosure Analysis . . . . . . . . . . . . . . . . . . . 18

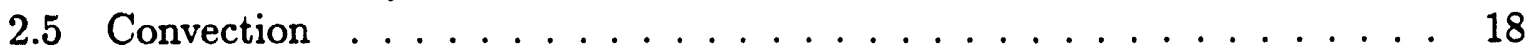

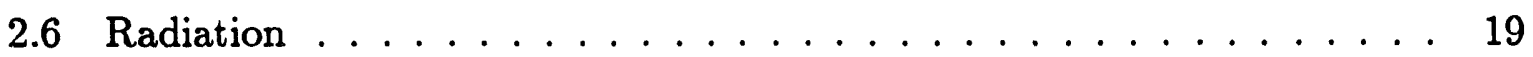

2.6.1 Radiating Enclosure Theory . . . . . . . . . . . . . 19

2.6.2 View Factor Estimation . . . . . . . . . . . . . . . . . 21

2.7 Cargo Model . . . . . . . . . . . . . . . . . . . . 23

2.8 Failure Criteria . . . . . . . . . . . . . . . . . 27

2.9 Numerical Methods . . . . . . . . . . . . . . . . . 27

3 Case Studies $\quad 30$

3.1 Case I . . . . . . . . . . . . . . . . . . . . . . 30

3.2 Case II . . . . . . . . . . . . . . . . . . . . 32

3.3 Case III . . . . . . . . . . . . . . . . . . . . . . 34

3.4 Case IV . . . . . . . . . . . . . . . . . . . . 34

4 MELTER Code Description $\quad 38$

4.1 Introduction . . . . . . . . . . . . . . . . . 38

4.2 Overview of Code Operation . . . . . . . . . . . . 38

4.3 Input . . . . . . . . . . . . . . . . . . . . 39

4.3 .1 scenes.BRC . . . . . . . . . . . . . . . 39

4.3 .2 cargo.TAR ........................ 41

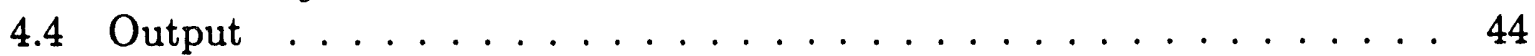

5 MELTER's Performance in PRA $\quad 47$

5.1 Numerical Uncertainty . . . . . . . . . . . . . . . . . . . 48

5.2 Distance . . . . . . . . . . . . . . . . . . 48

5.3 Fire Diameter . . . . . . . . . . . . . . . . . . 48 
5.4 SST Damage . . . . . . . . . . . . . . . . . 50

5.4 .1 Stored Energy Discharge . . . . . . . . . . . 50

$5.4 .2 A_{8}$ Nonuniformity . . . . . . . . . . . . . . 50

5.5 Cargo Damage . . . . . . . . . . . . . . . . 52

6 Concluding Remarks $\quad 53$

7 References $\quad 54$

A Parameter Optimization for the Charring Wall Model 55

B MELTER. OUT File for Case I 62

C Source Code Listing $\quad 65$

$\begin{array}{ll}\text { Distribution } & 95\end{array}$ 


\section{List of Figures}

1 Schematic representation of the most general configuration modeled. . . 3

2 Geometry to define fire boundary conditions. . . . . . . . . . . 7

3 View factor formula from Reference [3]. . . . . . . . . . . . . . 10

$4 A_{7}, F_{A_{7}, f}$, and $A_{5}$ versus separation distance for several fire diameters. . 13

5 Schematic of burning wall model. . . . . . . . . . . . . 16

6 Schematic of burning floor model. . . . . . . . . . . . . . . 17

7 Combined radiation and convection heat flux between planes. . . . . . . 20

8 Schematic representation of the cargo model for which ncs=5. . . . . 24

9 Temperature-time failure criteria used in MELTER and four hypothetical cargo responses. . . . . . . . . . . . . . . . 28

10 Schematic representation of geometries for four case studies. . . . . . . 31

11 Case I results. . . . . . . . . . . . . . . . . . . . . . 33

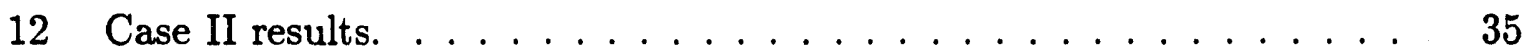

13 Case III results. . . . . . . . . . . . . . . . . . 36

14 Case IV results. . . . . . . . . . . . . . . . . . . 37

15 Example of two geometries that illustrate a model limitation. . . . . . . 51

16 Temperature histories from 2nd SST wall panel burn test. . . . . . . . 56

17 Temperature averages from 2nd SST wall panel burn test. . . . . . . . 57

18 Comparison of data and wall model using parameters optimized to the first 2000 seconds of data. . . . . . . . . . . . . . . . . . 59

19 Comparison of data and wall model using parameters optimized to the first 5000 seconds of data. . . . . . . . . . . . . . . . . 60

20 Comparison of data and wall model through 5000 seconds with parameters optimized to the first 2000 seconds of data. . . . . . . . . . 61 


\section{List of Tables}

1 Summary of cases considered. . . . . . . . . . . . . . . . . . 30

2 Portion of MELTER that reads scenes.BRC to define scenario parameters. . 40

3 Code section that reads cargo.TAR. . . . . . . . . . . . . 42

4 Code section that writes results. . . . . . . . . . . . . 46

5 DPTRA.BRC, the scenes.BRC file used to defined scenarios considered in results reported in Table $6 . \ldots \ldots$. . . . . . . . . . . . . 47

6 Summary of $\%$ of adjacent scenarios not exhibiting expected trend. . . . 49

7 Results of optimum wall parameter search. . . . . . . . . . . . 58 


\begin{tabular}{|c|c|c|}
\hline$A$ & area & $m^{2}$ \\
\hline$a_{w}$ & absorptivity of charred foam in diffusion model & $m^{-1}$ \\
\hline$C$ & specific heat & $J / k g / K$ \\
\hline$d$ & fire separation distance & $m$ \\
\hline$D$ & fire diameter & $m$ \\
\hline$F$ & view factor & Dimensionless \\
\hline$F_{A_{7}, f}$ & view factor from $A_{7}$ to fire & Dimensionless \\
\hline$h$ & layer thickness & $m$ \\
\hline & height & $m$ \\
\hline & convection coefficient & $W / m^{2} / K$ \\
\hline$k$ & thermal conductivity & $W / m / K$ \\
\hline$l_{w}, l_{f}$ & wall and floor foam thicknesses & $m$ \\
\hline$[P]$ & $\begin{array}{l}\text { matrix of radiation enclosure } \\
\text { properties }\end{array}$ & Dimensio: less \\
\hline$q$ & heat flux & $W / m^{2}$ \\
\hline$Q$ & heat transfer rate & $W$ \\
\hline$R$ & fire radius & $m$ \\
\hline$T$ & temperature & $K$ \\
\hline$x$ & spatial coordinate & $m$ \\
\hline$W, H, L$ & SST width, height, \& length & $m$ \\
\hline \multicolumn{3}{|l|}{ Greek } \\
\hline$\alpha$ & thermal diffusivity & $m^{2} / s$ \\
\hline$\beta$ & illuminated cargo fraction & Dimensionless \\
\hline$\beta, \theta$ & angle between surface and ray & Dimensionless \\
\hline$\delta_{i j}$ & Kronecker delta & Dimensionless \\
\hline$\Delta E_{\text {gassify }}$ & energy to gassify foam & $J / k g$ \\
\hline$\epsilon$ & emissivity & Dimensionless \\
\hline$\eta, \omega$ & conduction \& radiation connectors & $W / K$ \\
\hline$\ddot{\phi}, \theta$ & temperature predictions & $K$ \\
\hline$\rho$ & density & $\mathrm{kg} / \mathrm{m}^{3}$ \\
\hline$\rho C h$ & thermal capacitance per area & $J / m^{2} / K$ \\
\hline$\sigma$ & Stefan Boltzman constant & $W / m^{2} / K^{4}$ \\
\hline$\tau$ & transmitance & Dimensionless \\
\hline
\end{tabular}




\begin{tabular}{ll} 
subscripts & \\
\hline $1-9$ & refer to areas of enclosure problem \\
$f$ & designates quantity associated with fire \\
$A_{4 . .6}$ & refers to areas 4 through 6 \\
$f$ & Fire \\
$A_{7}, f$ & from $A_{7}$ to fire, for example \\
& $F_{A_{7}, f}=$ is view factor from $A_{7}$ to fire \\
$\infty$ & ambient condition \\
$p w$ & plywood \\
$s$ & surface
\end{tabular}

Fonts

Most of the theory in this document is discussed with the symbols previously listed. The type-writer font is used to make reference to variable names as they appear in the computer code implementation of MELTER. 


\section{Introduction}

Sandia National Laboratories (SNL) was the lead agency in conducting a study to assess the risk associated with ground transportation of radioactive defense program materials. A significant part of the risk is associated with a fire environment that might result from a traffic accident. A physical model, which has inherited the name MELTER, of the thermal response of cargos inside a Safe Secure Trailer (SST) subjected to a fire environment was developed. This report describes MELTER's underlying theory and information essential to the user of the associated code.

MELTER performs a transient thermal analysis of a SST and its cargo subjected to a fire boundary condition. Three sources of information determine conditions modeled in a given analysis:

\section{Properties of the SST.}

2. Properties defining the SST's cargo.

3. Scenario-specific parameters defining the fire boundary conditions and the condition of the SST and its cargo.

Properties defining each unique cargo considered in the risk assessment were derived once per cargo. Since there are a number of different cargos, MELTER must permit a convenient means of specifying the unique characteristics of each cargo. Once this information is developed it is effectively a data module which, along with charactei istics of the SST embodied in the code, defines the system to be subjected to boundary conditions defined by the probabilistic risk assessment (PRA). Selection of scenarios used in the PRA, definition of individual cargo characteristics, and post-processing of MELTER results to assess risk are described elsewhere.

MELTER has been developed with an effort to be balanced in the level of detail attempted in the analysis. Descriptions of scenarios (boundary conditions, really), free of delusive detail, are coarse. As such, the modeling of a response to these conditions to extreme detail is not justified. Furthermore, along with detail comes computational intensity. An affordable level of computational intensity has been struck with MELTER with most systems being represented by 15-21 degrees of freedom (variables) for most scenarios.

Section 2 includes a discussion of the underlying theory. Subsections cover the treatment of the fire radiation boundary condition, the charring SST wall, the transient thermal radiation enclosure analysis, the representation of cargos, and the numerical methods needed to render solutions. Section 3 presents results that are representative of the thermal response of the SST and its cargo for several scenarios of interest. Section 4 is devoted to describing the inputs needed to utilize MELTER. Section 5 assesses MELTER's 
performance based on results from its use in the PRA. Section 6 offers conclusions and assesses the strengths and weaknesses of MELTER.

Many simplifying assumptions are made to keep the model tractable including:

- All fires are represented by radiatively black, right circular cylinders. (Further consideration is given fires that are partly under the SST, see Section 2.2.)

- The SST wall response to fire is approximated with a simple, 3-variable model that represents foam decomposition with a planar interface dividing the charred and uncharred foam regions.

- Radiant heat transfer within the enclosure is between, at most, nine uniform surfaces.

- Convection is not considered on the interior or exterior of the SST.

- All cargos are represented by a spherically-symmetric geometry using a very limited number of state variables (temperature nodes) to represent thermal response.

- Except for allowance for a melting exterior, the thermal impedances of materials presumed to decompose within cargos are omitted rather than dealing with the decomposition processes.

Appendices include information too detailed to be included in the body of the report. The analysis leading to parameter selection characterizing the thermal response of the SST wall is documented in appendix A. Appendix B includes an optional output file produced by MELTER. Appendix $C$ includes a listing of the FORTRAN source code for MELTER. 


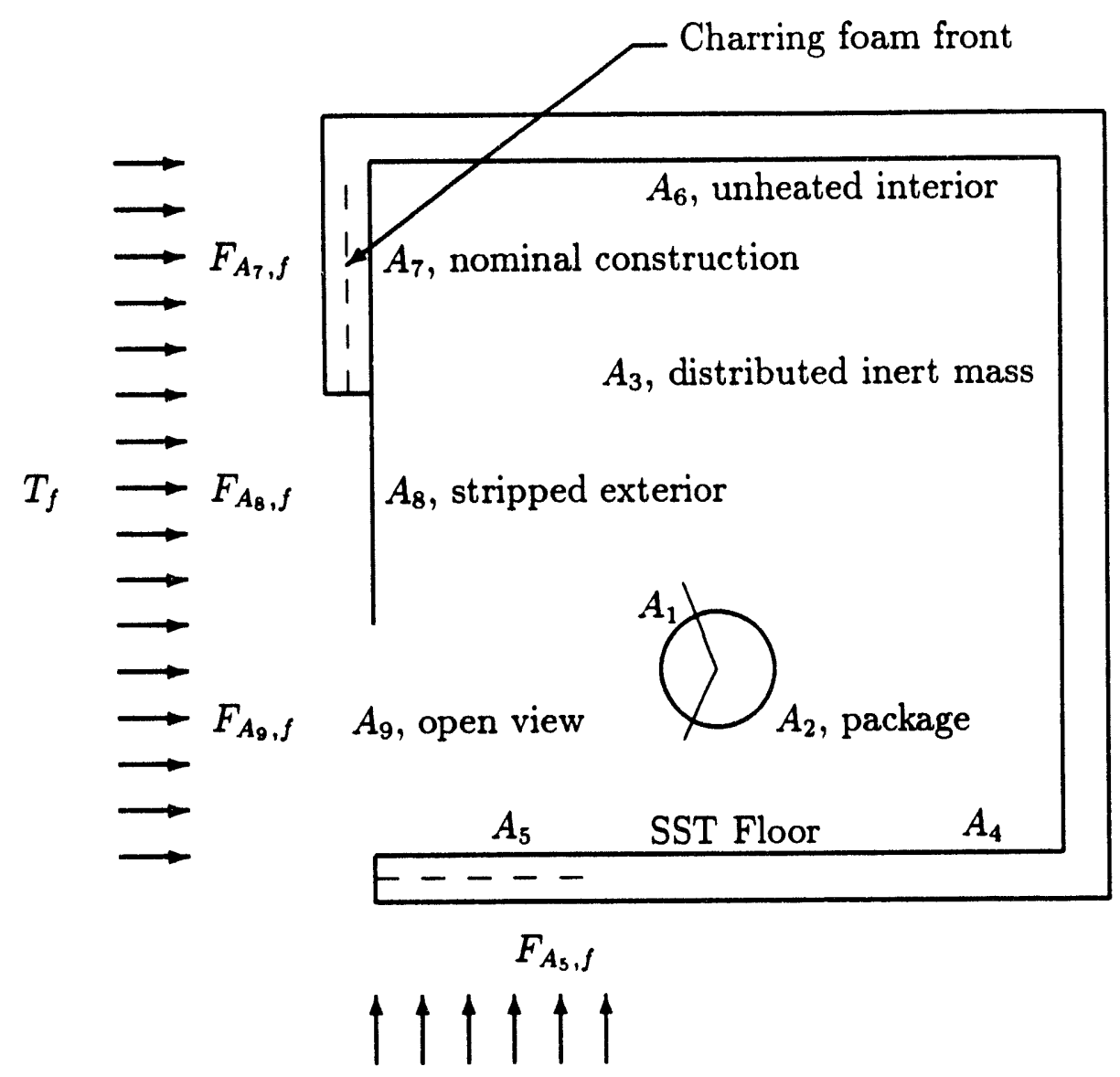

Figure 1: Schematic representation of the most general configuration modeled.

\section{MELTER Theory}

\subsection{Introduction}

Figure 1 presents an schematic overview of MELTER's underlying physical model. This figure represents the most complicated circumstance admitted by the model. In this section reference is occasionally made to variables by their names as they are implemented in the code. When these references occur the variable will be typeset in the typewriter font. The salient features of the model include:

1. At the heart of the system is a package whose thermal response is in question. The package's surface is partitioned into two areas designated $A_{1}$ and $A_{2} . A_{1}$ is some fraction of the cargo's total exterior area which is prorated to the fraction 
of the SST's exterior receiving direct heating by the fire. Associated with each of these areas are a temperature and surface emissivity. As described later, all cargos are represented by a spherical idealization.

2. The SST also includes inert mass which is important because it modifies the thermal response of the sensitive cargo. This inert mass represents various cargo within the interior walls of the SST. Treated as though it is dispersed throughout the volume, it is characterized by its total surface area, its total thermal capacitance, and a transmittance (transm) characterizing the view across the SST. ${ }^{1}$ The surface associated with this mass is designated as $A_{3}$. When the cargo in question consists of many identical copies, all but one threatened item will be lumped into the inert mass.

3. The floor is divided into areas $A_{4}$ and $A_{5} . A_{4}$ trades radiant energy with the rest of the enclosure, but that is its only means of changing temperature. $A_{4}$ is regarded as having thermal capacitance in communication with the SST's interior but insulated from the outside. $A_{5}$, on the other hand, is that portion of the floor subjected to a fire boundary condition.

4. The thermally massive interior walls of the SST are subdivided according to their exterior boundary conditions. That part of SST interior not being directly heated by the fire is designated as $A_{6}$. Conservatively, $A_{6}$ is assumed to be perfectly insulated on its exterior side.

5. $A_{7}$ is that portion of the SST's interior adjacent to an intact wall subjected to a fire boundary condition on its exterior.

6. $A_{8}$ is an area for which the armor interior is intact, but the exterior skin and foam insulation have been removed by mechanical damage to the SST and subjected to a fire boundary condition. Compared to $A_{7}$, this section offers less protection from the fire.

7. $A_{9}$ represents an opening on the surface of the SST with an open view of a fire.

Various scenarios result in corresponding distributions of $A_{1 . .9}$. All of $A_{1 . .3}$ and least one of $A_{5,7 . .9}$ are present in every scenario. The PRA requires representation of:

- the fire characteristics of diameter, separation distance, and temperature.

- the SST's state of repair represented by various values of $A_{7}, A_{8}$, and $A_{9}$.

\footnotetext{
${ }^{1}$ A clean, unattenuated, view has a transmittance of 1 while a value of 0 corresponds to complete obstruction.
} 
- as many as four damage states representing the condition of the cargo.

MELTER generates its solution to every combination of several discrete values of each of foregoing variables. From the fire characteristics of diameter, distance, and temperature MELTER develops the quantities represented in Figure 1 to represent the fire boundary condition. The interior surfaces $\left(A_{1 . .9}\right)$ exchange energy only by thermal radiation. The state variables of the model consist of a temperature for each of the $A_{1 . .8}$ present, temperatures representing locations in the cargo's interior, skin temperatures and char displacements for wall sections adjacent to $A_{5}$ and $A_{7}$, and the plywood/foam interface temperature associated with $A_{5}$. MELTER's end product is an estimation of the minimum duration a given fire geometry must have to induce a critical temperature in the cargo.

It is natural to consider this analysis from the fire inwards to the threatened cargo. Logical pieces of the analysis are:

- The fire boundary condition's representation.

- The wall model which is descriptive of the charring process that occurs in either the floor or the wall.

- The radiation enclosure problem associated which heat transfer inside the SST.

- The cargo model.

- Numerics associated with solving the model's equations.

Each of the these topics is addressed in the following subsections.

\subsection{Fire Boundary Condition}

\subsubsection{Fire Geometry}

MELTER allows fires to be of any displacement, size, or temperature and, in fact, these three variables constitute a complete fire description to MELTER. The complexity of gaseous radiation consideration is avoided by assuming unit emissivity for the fire. Since unit emissivity is a limiting value associated with large fires, the assumption can only be conservative which is consistent with the philosophy used throughout the model. $^{2}$

\footnotetext{
${ }^{2}$ Reference [1] deals with a more detailed treatment of the fire's shape and temperature distribution. While that study produced satisfying results, the spatial integrations through gray gaseous matter of a specified temperature distribution impose too much calculational detail to be included in the present model.
} 
A fire's displacement is the distance between its base's center and a point centered under the SST measured along a line that is a perpendicular bisector of the long side of the SST. Zero displacement corresponds to a fire centered beneath the SST.

$A_{5}$ is present in the model only for fires located at least partially beneath the SST. When the fire is not under the SST its geometry is presumed to be that of a right circular cylinder of a height three times the base diameter. ${ }^{3}$ The method of calculating $A_{1 . .9}$ and view factors between SST exterior surfaces and the fire are given in the following subsection.

\subsubsection{Inferred Parameters}

The primary aim of this section is to define the means MELTER uses to infer the more complete fire description from the limited input of separation distance, diameter, and temperature. Specifically, (refer to Figure 1) $A_{7}, A_{5}$, and $F_{A_{7}, f}$ must be systematically inferred from the inputs of fire distance and diameter. $F_{A_{5}, f}$ is always unity. The specification of $A_{8}$ and $A_{9}$ are not within the domain of MELTER. They are specified by the PRA analyst. In MELTER, when $A_{9}$ and $A_{8}$ are greater than zero they reduce $A_{7}$ in that order to determine SST exposure through these areas. By reducing $A_{7}$ in the order just specified, the most hazardous surface is given preferential access to the fire. For simplicity, the view factors $F_{A_{8}, f}$ and $F_{A_{9}, f}$ are taken to be the same as $F_{A_{7}, f}$.

The calculation of these quantities must consider all combinations of separation distance and fire diameter. For the present discussion let the SST have length, width, and height dimensions of $L, W$, and $H$; and the fire have separation distance, $d$; diameter $D$; and height, $h_{f}$. Reference to Figure 2 will be useful in the following discussion. The following list, ordered by separation distance, summarizes the methods of calculation used:

1. Fire center under SST $(0<d<W / 2)$.

(a) $A_{5}$ is the intersection of the circular fire base area $\left(\pi R^{2}\right)$ and the projection of the SST's area $(L \times W)$ onto the ground. A maximum corresponding to the whole bottom area of the SST is imposed.

(b) $A_{7}$ is calculated for the specified fire diameter, but the fire location is artificially presumed to be such that the center is immediately under one edge of the SST $(d \sim W / 2)$. (What actually happens when a fire is under the vehicle is, of course, complicated and dependent on many details left unspecified in the present analysis. Hence, the simplifying assumption.) See the next item for additional detail on this calculation.

\footnotetext{
${ }^{3}$ Reference [2] gives information pertinent to the aspect ratio of the fire. The aspect ratio $\left(h_{f} / D_{f}\right)$ grows slightly with increasing fire size. $h_{f} / D_{f}=3$ gives a conservatively tall fire for larger fires.
} 


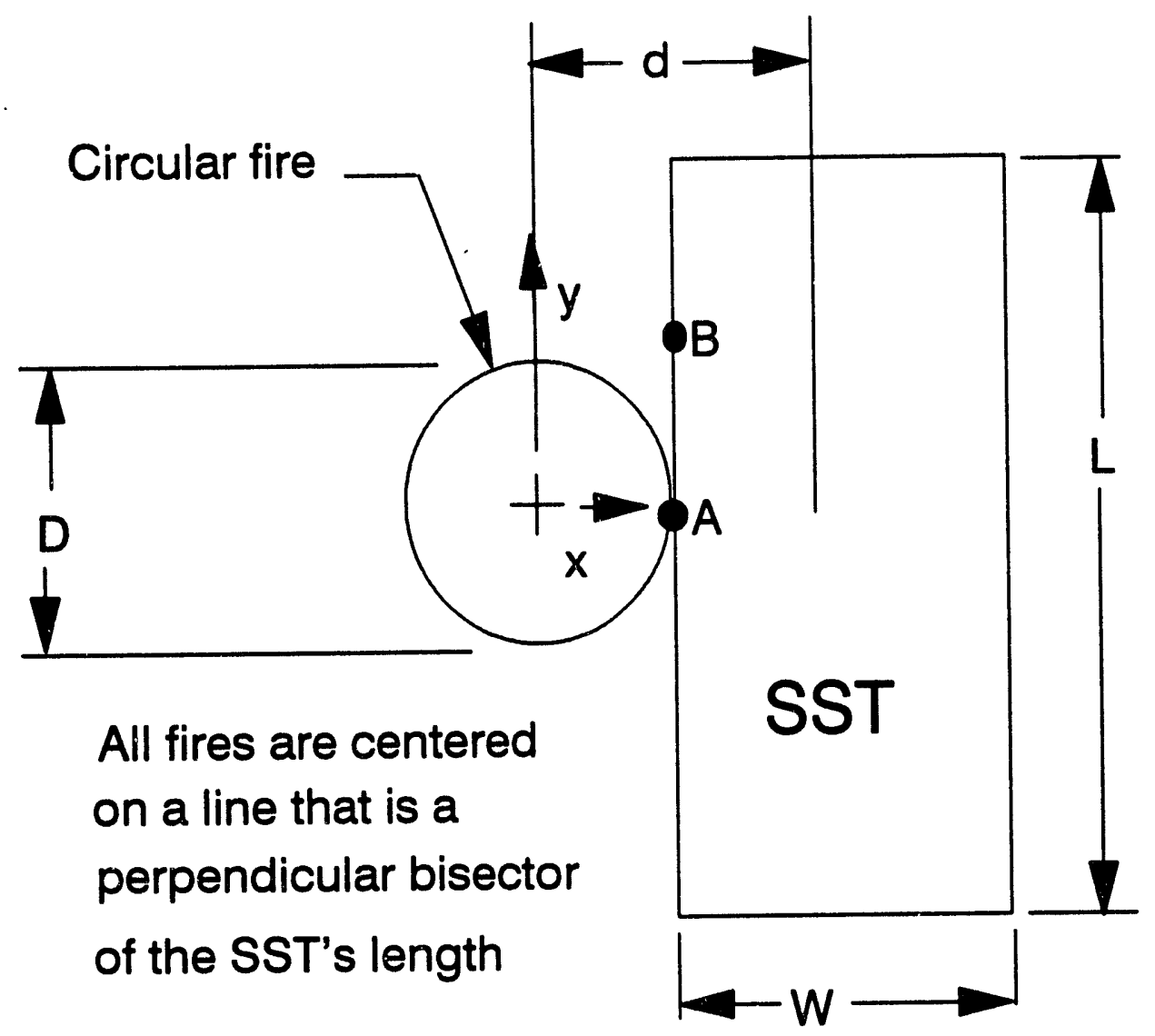

$z$ - direction is positive out of page and $z=0$ at ground level At " $\mathrm{A}$ " the view factor to the fire $=1$ at " $\mathrm{B}$ " it's about 0.2

Figure 2: Geometry to define fire boundary conditions. 
(c) $F_{A_{7}, f}$ is one.

2. Center out from under SST but fire extending under $(W / 2<d<W / 2+R)$.

(a) $A_{5}$ is the intersection of the circular fire base area $\left(\pi R^{2}\right)$ and the projection of the SST's area $(L \times W)$ onto the ground. A maximum corresponding to the whole bottom area of the SST is imposed.

(b) $A_{7}$ is at least an area corresponding to the height of the SST times the length of the chord formed by the intersection of the plane of the SST wall and the fire base circle. This area will generally be greater than that, the additional length being calculated in the manner described in the following subsection. If the fire diameter is such that the plane of the far side of the SST also intersects the base circle, that chord length is also considered. $A_{7}$ also includes area from the SST's roof in a manner proportional to the area calculated considering the vertical sides. That is, when the foregoing calculation indicates that all of the vertical wall surface is exposed, all of the ceiling area is also exposed. A maximum corresponding to all the vertical surface area of the SST plus the roof area is imposed.

(c) $F_{A_{7}, f}$ is one.

3. Fire clear of SST $(d>W / 2+R)$.

(a) $A_{5}$ is zero.

(b) $A_{7}$ for the vertical surfaces is determined by the somewhat lengthy process described in the following subsection. 'The SST's roof is prorated as described earlier.

(c) $F_{A_{7}, f}$ is the view factor between a right circular cylinder of the fire's dimensions and an element of area located on a radial from the fire center at a height half way up the side of the SST. This is described further in the following section.

\subsubsection{View Factors and Exposed Area}

This section describes a calculational procedure for specifying $A_{7}$ and $F_{A_{7}, f}$, when a fire diameter, height, and separation distance are specified. $A_{7}$ and $F_{A_{7} f}$ are the area of normal construction SST wall exposed to fire and the corresponding view factor to the fire. For computational expedience we are assuming that all fires are centered on a perpendicular bisector of the long side of the SST. The origin of the coordinate system is at the center of the base of the fire. The side of the SST facing the fire is 
perpendicular to the $x$-axis, the length of the SST is aligned with the $y$-axis, and the $z$-axis is perpendicular to the ground.

The following assumptions are made:

1. All fires are located along the perpendicular bisector of one side of the SST trailer.

2. All fires are right circular cylinders.

3. All fires are optically thick, so that the SST perceives the right circular cylinder as a solid, blackbody radiator.

In general, the view factors and areas between some plane area in the side of the SST and the fire are related by:

$$
A_{1} F_{1,2}=A_{2} F_{2,1}=\frac{1}{\pi} \int_{A_{1}} \int_{A_{2}} \frac{\cos \theta_{i} \cos \theta_{j}}{S_{i, j}^{2}} d A_{i} d A_{j}
$$

where $S_{i, j}$ is the path length between two area elements $A_{i}$ and $A_{j}$, and $\theta_{i}$ and $\theta_{j}$ are the angles between $S_{i, j}$ and a line normal to $A_{i}$ or $A_{j}$ respectively.

Siegel and Howell (Ref. [3]) give a formula for evaluating Eq. (1) when $A_{2}$ is an area element that is perpendicular to a line passing through one end of the axis of symmetry of a right circular cylinder. Let us denote that formula as $g(\lambda, \kappa)$ where $\lambda$ and $\kappa$ are as defined in Figure 3.

A more general formula may be formed for area elements not perpendicular to the aforementioned line passing through the base midpoint of the cylinder. That is:

$$
g^{*}\left(\lambda, \kappa^{*}\right)=\cos (\beta) g\left(\lambda, \kappa^{*}\right)
$$

where $\beta$ is the angle between a line normal to the area element and a line passing through the base center of the cylinder and the element. $\kappa^{*}$ is based on the distance from the element to the cylinder's centerline. For the coordinate system described earlier, $\kappa^{*}=2 x /(D \cos \beta)$, where $\beta=\tan ^{-1}(y / x)$. Finally, an area element on the side of the SST is not at the same $z$-coordinate as the fire base. To deal with this, we let the fire be two right circular cylinders, one measuring down from $z$-location of the area element and one measuring up (this assumes the fire is tall enough so that $h_{f}>z$, where $z$ corresponds to the area element). Then

$$
F_{d 1,2}=g^{*}[2 z / D, 2 x /(D \cos \beta)]+g^{*}\left[2\left(h_{f}-z\right) / D, 2 x /(D \cos \beta)\right]
$$

So for a particular $d, D$, and $h_{f}$, Eq. (3) can be integrated over some area in the plane of the SST's side to yield the view factor between the two finite areas. In the analysis of radiation exchange between surfaces, Eq. (1), is based on the idealization that thermal conditions be uniform over $A_{1}$ and $A_{2}$. For our analysis assuming constant 
Plane element $d A_{1}$ to right circular cylinder of finite length $l$ and radius $r$; normal to element passes through one end of cylinder and is perpendicular to cylinder axis.

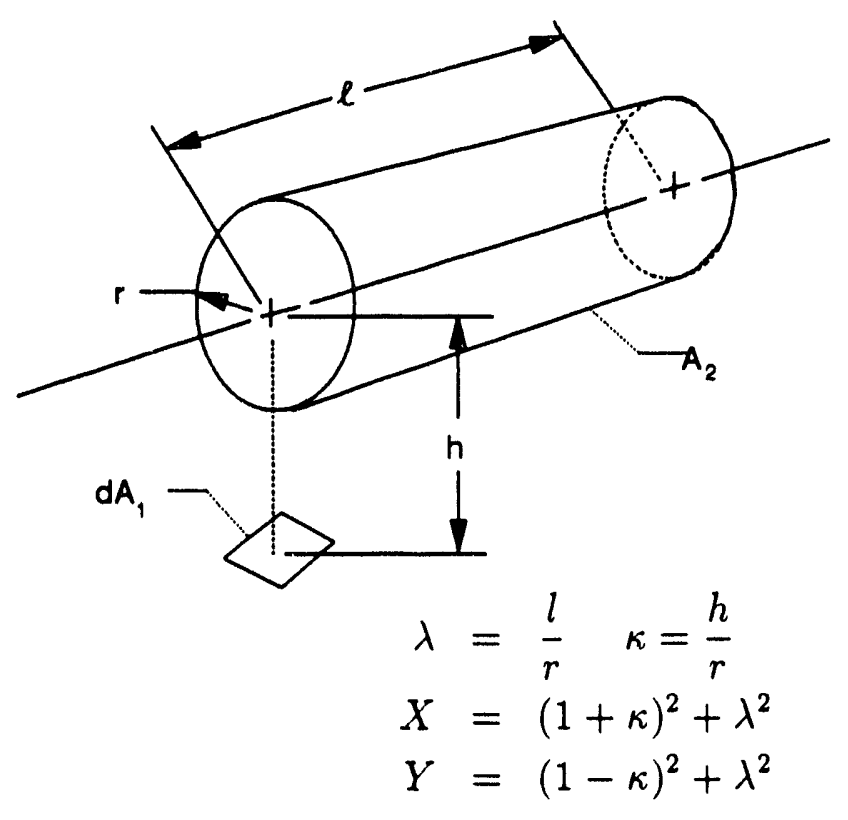

$$
\begin{aligned}
& g(\lambda, \kappa)=F_{d 1-2}= \\
& \frac{1}{\pi \kappa} \tan ^{-1} \frac{\lambda}{\sqrt{(\kappa)^{2}-1}}+ \\
& \frac{\lambda}{\pi}\left[\frac{X-2 \kappa}{\kappa \sqrt{X Y}} \tan ^{-1} \sqrt{\frac{X(\kappa-1)}{Y(\kappa+1)}} \frac{1}{\kappa} \tan ^{-1} \sqrt{\frac{(\kappa-1)}{(\kappa+1)}}\right]
\end{aligned}
$$

Figure 3: View factor formula from Reference [3]. 
conditions over $A_{1}$ (the fire) is reasonable. Uniformity over $A_{2}$, the SST side, is clearly an idealization for convenience. Imagine, for example, the situation depicted in Figure 2. Clearly the conditions are not uniform over $A_{2}$. This subsection deals with defining an appropriate viewfactor and finite area to represent the fire.

We need a means to pick $A_{7}$ and $F_{A_{7}, f}$, the area and view factor of normally constructed wall exposed to the fire. If the circumstance modeled is to include $A_{8}$ or $A_{9}$ areas they will be deducted from the calculated $A_{7}$. Consider some subarea on the side of the SST that can see both a fire and a cooler surrounding temperature. That area element will be its hottest if it is not losing heat through the wall, but is in radiative equilibrium (we're ignoring convection here). Under those conditions, its equilibrium emissive power, $E$, is given by:

$$
E=E_{\infty}+F \times\left(E_{f}-E_{\infty}\right)
$$

where $F$ is the local view factor to the fire. For the conditions of Figure 2, the emissive power at $x=d, y=0, z=\bar{h}_{s s t}$ is equal to that of the fire, where $\bar{h}_{s s t}$ is the the $z$-value half way up the SST's wall. At $x=d, y=d, z=\bar{h}_{s s t}$ the view factor is only 0.19 for a tall fire and the corresponding decrease in emissive power can be seen from Eq. (4). Clearly, letting the view factor be 1 and the area, $A_{7}$, the whole side of the SST is too ccnservative. There is a whole two-dimensional field of $E(x=d, y, z)$ (and corresponding view factor variation) on the side of the SST. The task at hand is to pick single values of $A_{7}$ and $F_{A_{7}, f}$ that realistically approximate the real conditions.

Consider conditions similar to those of Figure 2. If we choose $A_{7}$ to be large, then much of it must lie in areas for which the view factor should be comparatively small. On the other hand, if $A_{7}$ is chosen to be comparatively small, we might miss significant energy deposition on the side of the SST. Equations (3) and (4) can be used to estimate the emissive power of locations on the heated surface for conditions of radiative equilibrium. ${ }^{4}$ That is:

$$
\begin{aligned}
E(x=d, y, z)= & E_{\infty}+\left(E_{f}-E_{\infty}\right) \times \\
& \left\{g^{*}[2 z / D, 2 x /(D \cos \beta)]\right. \\
& \left.+g^{*}\left[2\left(h_{f}-z\right) / D, 2 x /(D \cos \beta)\right]\right\}
\end{aligned}
$$

Now we may use the preceding equation to consider how far $A_{7}$ should extend. We know that the foam construction of the wall will decompose only very slowly below a temperature of around $585 K$ (Refs. [4,5]). Clearly, energy from the fire that lands on some surface not achieving an important temperature rise is of little consequence.

\footnotetext{
${ }^{4}$ This leads to a conservatively high estimate in the current case because our surface experiences heat loss through the wall and/or to ambient air.
} 
Since the SST is a lot longer than it is tall, it is sensible to make life still a bit easier by looking at the emissive power on the wall only at one value of $z$, say $\bar{h}_{s s t}$. The following means of setting $A_{7}$ is used:

$$
E_{\text {max }} A_{7}=2 \int_{0}^{y^{*}} E\left(x=d, y, z=\bar{h}_{s s t}\right) H d y
$$

where $H$ is the vertical length of the SST side. $y^{*}$ is the lesser of half the SST's length and that value of $y$ for which $E\left(x=d, y, z=\bar{h}_{s s t}\right)=\sigma T_{f}^{4}$ after which $E$ is too low to threaten the wall construction. $E_{\max }$ is the value of $E$ at $y=0$ and the factor of 2 comes from the fact the problem has symmetry about the $(y=0)$-plane. Notice that the area of integration on the right hand side is greater than $A_{7}$. Recognizing that the background emissive power is negligible compared to that of the fire, the $E$-distribution (for our adiabatic wall) is the same as the distribution of incident radiant energy. Then Eq. (6)'s right hand side is the summation of all the energy that is deposited on the side of the SST in such concentration as to produce local temperatures that will result in foam decomposition. $A_{7}$ is then chosen so that the aforementioned quantity of energy is preserved, but it is all to be delivered at the maximum intensity. This a convenient means of defining the extent of $A_{7}$. Furthermore, it's conservative. For an actual fire of some practical duration and approximating the geometry depicted in Figure 2, the effect of the fire will be most severe along $y=0$. At some larger value of $y$ there will begin areas which are only partly burnt through. These areas, while absorbing energy from the fire, will not contribute significantly to the overall heat transmission into the SST's interior. This approach takes this same energy and concentrates it to an intensity that is much more effective at burning its way into the SST's interior.

Figure 4 includes plots of $A_{7}, F_{A_{7}, f}$, and $A_{5}$ versus separation distance for fire diameters of $1,2,4,8$, and 16 meters.

\subsection{Charring Foam Wall Model}

With reference to Figure 1 , the areas $A_{5}$ and $A_{7}$ correspond to floor and wall area of nominal construction subjected to a fire boundary condition. A simplified model, as described in this section, is utilized to represent the significant impediment to heat transfer represented by the foamed wall/floor construction. The form of the model governing the charring of the foam is identical for the wall and floor locations.

\subsubsection{Wall Model}

The foamed wall of the SST plays an important role in many potential fire scenarios. Fire tests on SST wall sections directly adjacent to fire have demonstrated: 


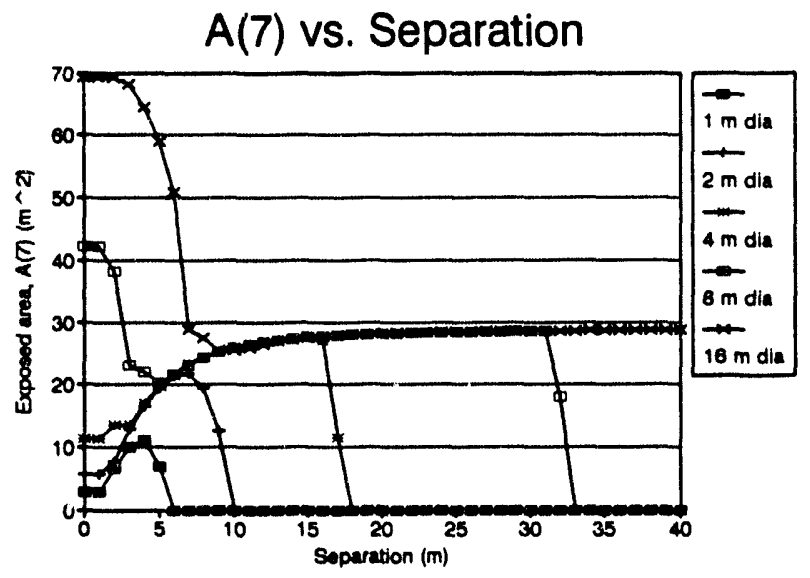

\section{View Factor vs. Separation Distance}

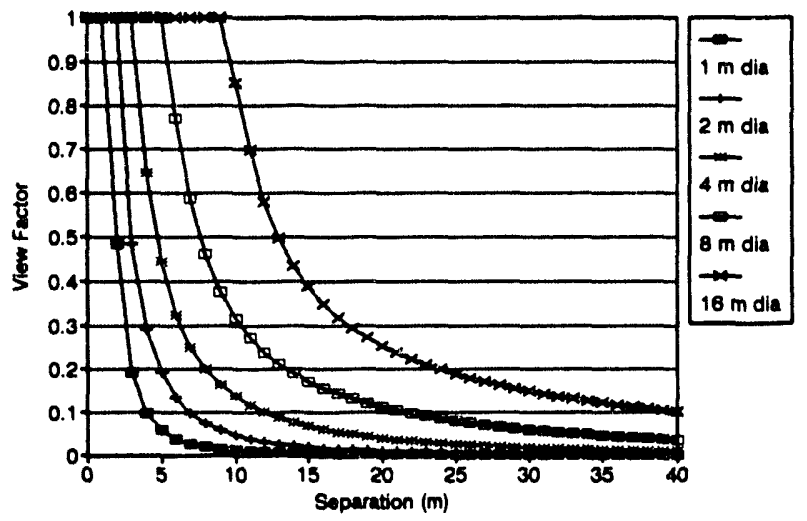

\section{Exposed Floor Area vs. Separation}

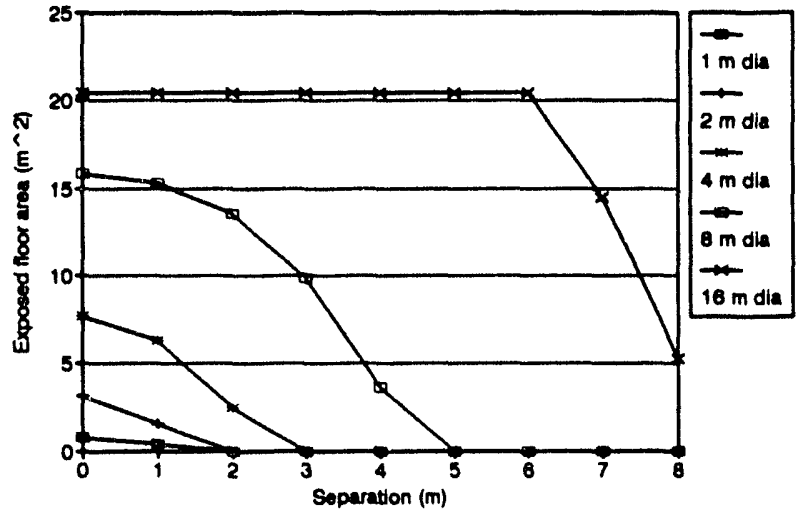

Figure 4: $A_{7}, F_{A_{7}, f}$, and $A_{5}$ versus separation distance for several fire diameters. 
1. There is virtually no heat transfer through the wall until the foam is decomposed. Most locations on the wall interior show no response for perhaps 10 minutes after the start of a directly-impinging fire.

2. The behavior of the wall burning process varies from test to test and from location to location on a given test.

3. After the wall's foam is completely charred, the residue is of very low thermal capacitance, but offers a significant impediment to radiation heat transfer through the wall.

As we wish to represent this complicated process with a simple model, it is isconcerting that the composition of the SST wall is not uniform following a fire test. Obviously, the resultant residue of a fire is dependent on many parameters-more than can be realistically included in our modeling effort. Nonetheless, some sort of simplified wall model must be present to give a fair representation of the SST's response under many circumstances. It is not actually the wall's temperature response we are concerned about, but rather the degree to which the wall impedes heat transfer into the SST's interior. In the tests that were conducted (Ref. [6]) conditions were severe enough to guarantee the hasty decomposition of the foam. A matter of considerable concern is the prediction of the wall's behavior when the fire boundary condition is less severe. If the fire is distant enough there exists some point at which the foam in the wall will experience no significant deterioration and there will be effectively no heat transfer through the wall for hours.

It seems apparent that great detail in resolving temperature profiles through the wall is not justified. Moreover, it's not even particularly useful-all we really care about is the response of the SST's interior. In order to eliminate needless number-crunching we seek an approximate model of the wall's behavior which will yield satisfactory results. Figure 5 shows the simplified wall model schematically. In the simplified model the state of the wall is characterized by its two surface temperatures and the position of the decomposition front:

1. The exterior skin's temperature's $\left(T_{s}\right)$ derivative is given by:

$$
\frac{\partial}{\partial t} T_{s}=\frac{1}{(h \rho C)_{s}}\left(q_{f}-q_{\text {rad }}\right)
$$

where,

$$
q_{f}=\sigma \epsilon_{s}\left\{F_{A_{7}, f}\left(T_{f}^{4}-T_{\infty}^{4}\right)+T_{\infty}^{4}-T_{s}^{4}\right\}
$$

and

$$
q_{\text {rad }}=\frac{\sigma\left(T_{s}^{4}-T_{\text {react }}^{4}\right)}{1+\frac{3 a_{w} x}{4}}
$$


$a_{w}$ is the absorption coefficient for radiation in the charred region, and $T_{\text {react }}$ is a constant temperature at which the foam is assumed to gassify.

Equation (9) is the steady heat transfer rate through an optically thick medium between radiatively black plates (Reference [3]). $T_{\text {react }}$ (Eq. (9)) is replaced by the temperature of $A_{7}$ when $x=l_{w}$.

2. $x$ is the position of the char front. At the start of the analysis $x=0$ and during the analysis the velocity of the front is given by:

$$
\frac{\partial x}{\partial t}=\frac{q_{\text {rad }}}{\rho_{w} \Delta E_{\text {gassify }}}
$$

until $x=l_{w}$ and zero thereafter. To conserve energy, $q_{w a l l}$, the energy conducted from the reaction front inwards to the armor, should be deducted from the numerator of Eq. (10). However, due to the simple definition of $q_{\text {wall }}$, the front could not advance all the way to the armor. Consequently, the wall is allowed to "create" this insignificant amount of energy for numerical expedience.

3. The interior wall $\left(T_{A_{7}}\right)$ temperature is found by integrating:

$$
\frac{\partial}{\partial t} T_{A_{7}}=\frac{1}{(h \rho C)_{7}}\left(q_{w a l l}-q_{7}\right)
$$

4. In Eq. (11), $q_{\text {wall }}=q_{\text {rad }}$ as predicted by Eq. (9) after $x=l_{w}$ and $q_{\text {wall }}=$ $\left(T_{\text {react }}-T_{A_{7}}\right) k_{w} /\left(l_{w}-x\right)$ for $x<l_{w}$, but not allowing $q_{w a l l}>q_{\text {rad }} . q_{7}$ is the heat flux into the SST from $A_{7}$ as determined by the enclosure analysis defined in a subsequent section (see Eq. (18)). $k_{w}$, treated as constant, is the thermal conductivity of the foam before charring.

The parameters $a_{w}, \Delta E_{\text {gassify }}$, and $T_{\text {react }}$ have important influences on the wall model's response. A study was conducted to choose these parameters to make the model agree with the best available test data. That study is incorporated as Appendix A.

\subsubsection{The Charring Floor Model}

This model is associated with $A_{5}$ identified earlier in Figure 1. Figure 6 is a schematic of the burning floor model which shows the layer of plywood present in the floor construction. Equations identical to (7-10) are used to characterize the adjacent skin temperature and char displacement. In tests associated with the floor it has been demonstrated that the plywood layer is a significant impediment to heat transfer. As a 


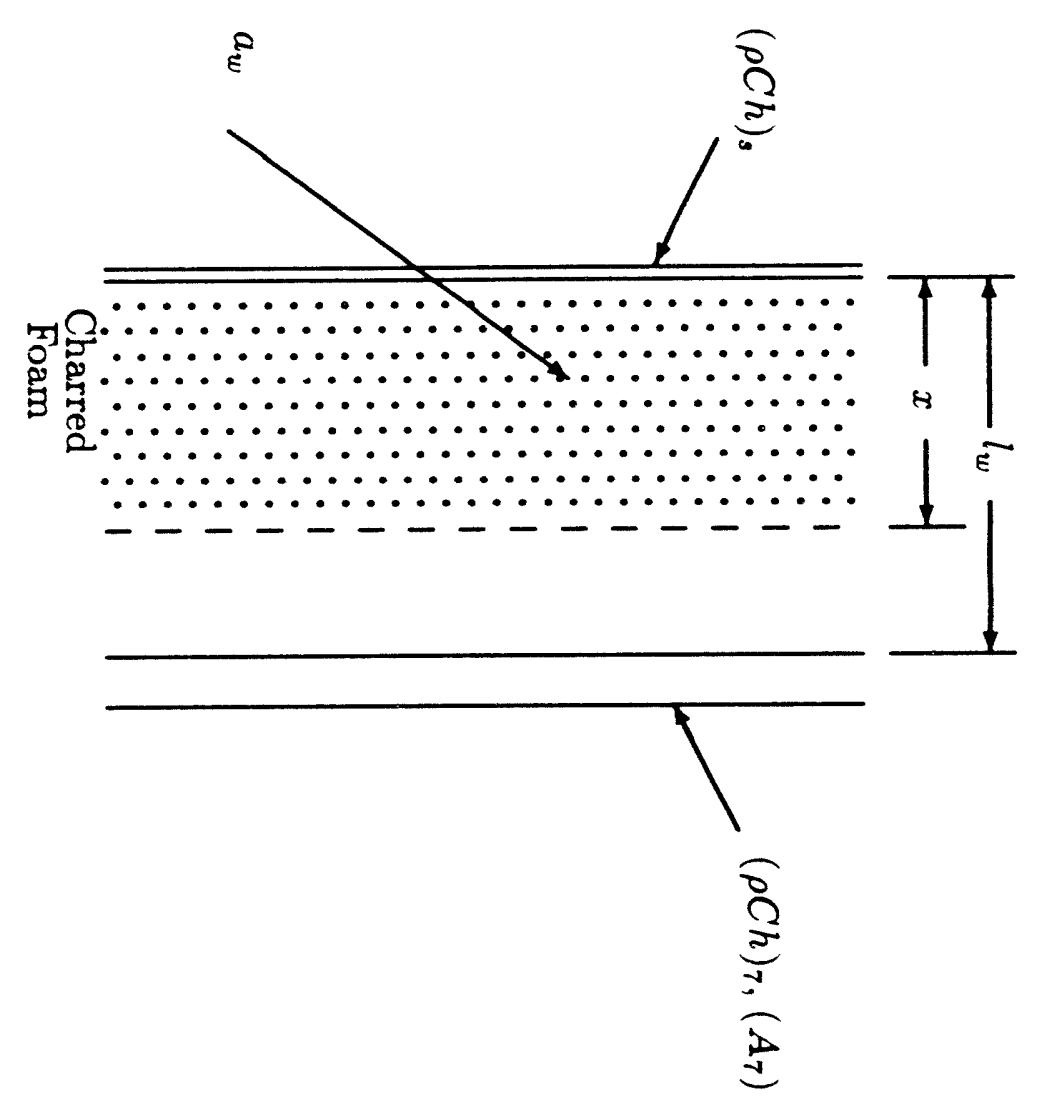




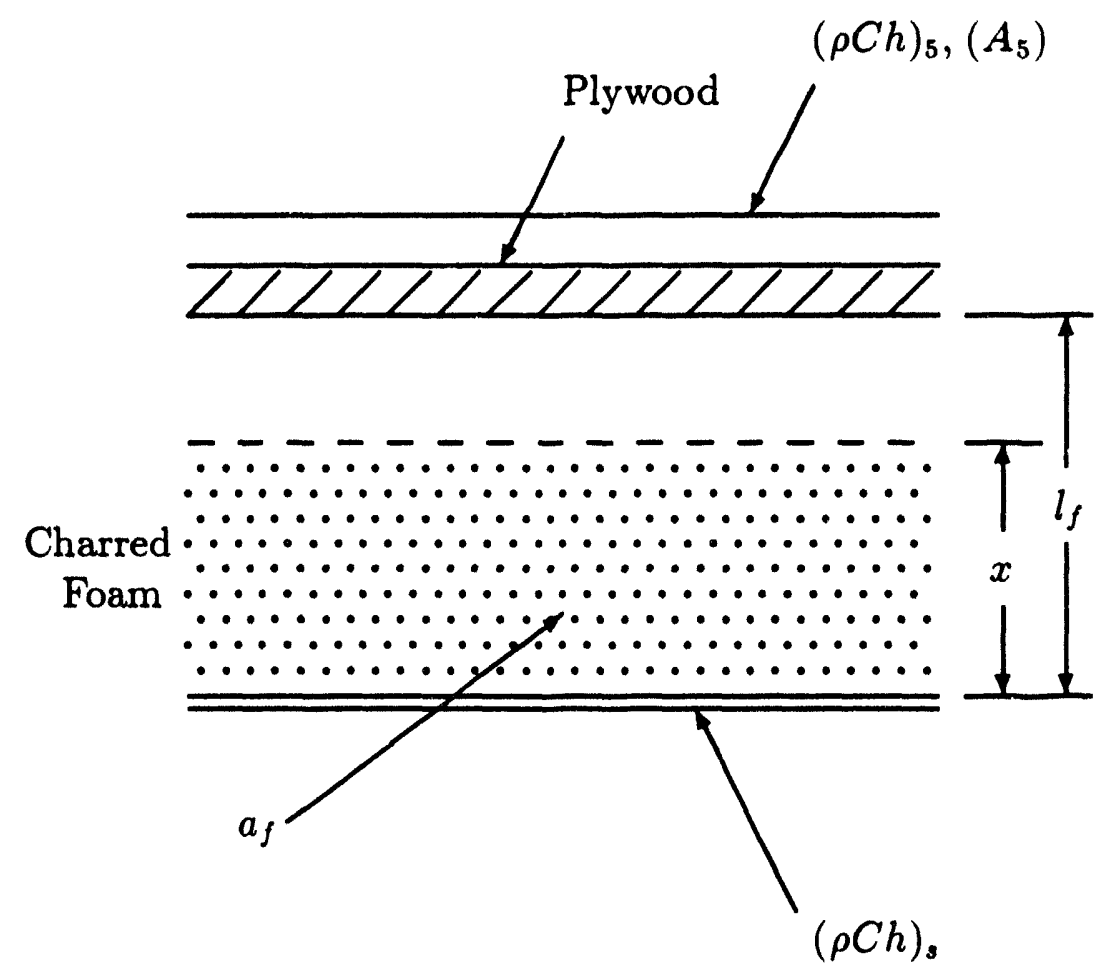

Figure 6: Schematic of burning floor model. 
result, corresponding to Eq. (11) of the wall model are two equations associated with the floor's plywood and metal temperatures.

$$
\begin{gathered}
\frac{\partial}{\partial t} T_{p w}=\frac{1}{(h \rho C / 2)_{p w}}\left(q_{w a l l}-q_{p w}\right) \\
\frac{\partial}{\partial t} T_{A_{5}}=\frac{1}{(h \rho C / 2)_{p w}+(h \rho C)_{5}}\left(q_{p w}-q_{5}\right)
\end{gathered}
$$

In the preceding equations $q_{p w}$ is the conduction heat flux through the plywood given by:

$$
q_{p w}=\frac{k_{p w}}{h_{p w}} \times\left(T_{p w}-T_{A_{5}}\right)
$$

In Eq. (12) the term $(h \rho C / 2)_{p, w}$ is half the thermal capacitance associated with the plywood. The other half is grouped with that associated with the metal of the floor (Eq. (13)). This partitioning of the plywood's thermal capacitance is appropriate since it is much less thermally diffusive than the adjacent metal. Thermal properties of virgin plywood are used throughout the analysis. As with the wall model, $q_{5}$ is determined by the enclosure analysis of the following section. $q_{\text {wall }}$ is also calculated as described for the wall model.

An analysis similar to that for the charring of the foam might have been incorporated to represent the charring of the plywood. Since we expect the plywood charring to act as a heat sink and the thermal resistance of the plywood to increase as it is charred, the present approach is conservative.

\subsection{Enclosure Analysis}

Thermal radiation is the dominant mechanism for transport of energy across the SST enclosure. In fact, convection and conduction are not considered. While the armor is a fair thermal conductor, long path lengths associated with energy movement through the SST result in slow conduction responses. Further, the threatening fire environments give rise to significantly elevated temperatures at which radiation can be expected to dominate (Ref. [7]).

\subsection{Convection}

Figure 7 is included to illustrate the relative importance of convection across the enclosure. The physical system considered in Figure 7 is comprised of two parallel planes. The space between the planes is filled with air which is transparent to the thermal radiation but contributes to the total heat flux by convection. The significance of the 
convection is estimated by:

$$
q_{\text {conv }}=h(T)\left(T_{1}-T_{2}\right) / 2.0
$$

where

$$
h(T)=1.31\left\{\left(T_{1}-T_{2}\right) / 2\right\}^{1 / 3}
$$

Eq. (16) is a simplified expression for $h$ for turbulent natural convection between air and a vertical surface as given by Reference [8]. $h$ is expressed in $W / m^{2} / K$ and $T_{1}-T_{2}$ in $K$. The radiation is estimated from:

$$
q_{r a d}=\sigma \frac{\left(T_{1}^{4}-T_{2}^{4}\right)}{1 / \epsilon_{1}+1 / \epsilon_{2}-1}
$$

In the present discussion $T_{1}$ is simulating one of the directly heated surfaces shown in Figure 1. One surface temperature, $T_{1}$, is varied from 300 to $1500 \mathrm{~K}$ while the other, $T_{2}$, is held constant at $300 \mathrm{~K}$. The total heat flux between the planes and fraction of it due to convection are shown in Figure 7 over the range of $T_{1}$. As $T_{1}$ reaches threatening temperatures the total heat transfer is dominated by radiation. The convection estimate assumes the same $h$ at the hot and cold walls so that the air temperature is assumed to be the average of $T_{1}$ and $T_{2}$. That is, this simple analysis is making the conservative assumption that the air is always well mixed.

\subsection{Radiation}

\subsubsection{Radiating Enclosure Theory}

The thermal radiation model used can be described in terms of a series of discrete surface areas forming an enclosure. This theory, widely used, includes some very limiting assumptions including: emissivity independent of wavelength, uniform properties over each enclosing surface identified, and diffuse emission and reflection. All of these assumptions are frequently made in engineering analyses to arrive at a tractable formulation. The poorest of these assumptions in the current context is undoubtedly the uniform conditions across a given surface. The equations that are used to solve the radiating enclosure problem are linear in the emissive powers of the enclosure surfaces. Their precise form varies in texts on the topic so the approach used in the current investigation is stated briefly here. Given the emissivities and surface temperatures of an $N$-surface enclosure the heat fluxes can be summarized tersely as:

$$
\bar{q}=\{[F]-[I]\}[P]^{-1} \bar{b}
$$

In the preceding equation: $[F]$ is the $N \times N$ matrix of the view factors between the surfaces and $[I]$ is the identity matrix. $[P]$ is the square matrix whose elements are, 


\section{Total Heat Flux \& \%-Convected vs. T_1}

Radiation/Convection Between Planes
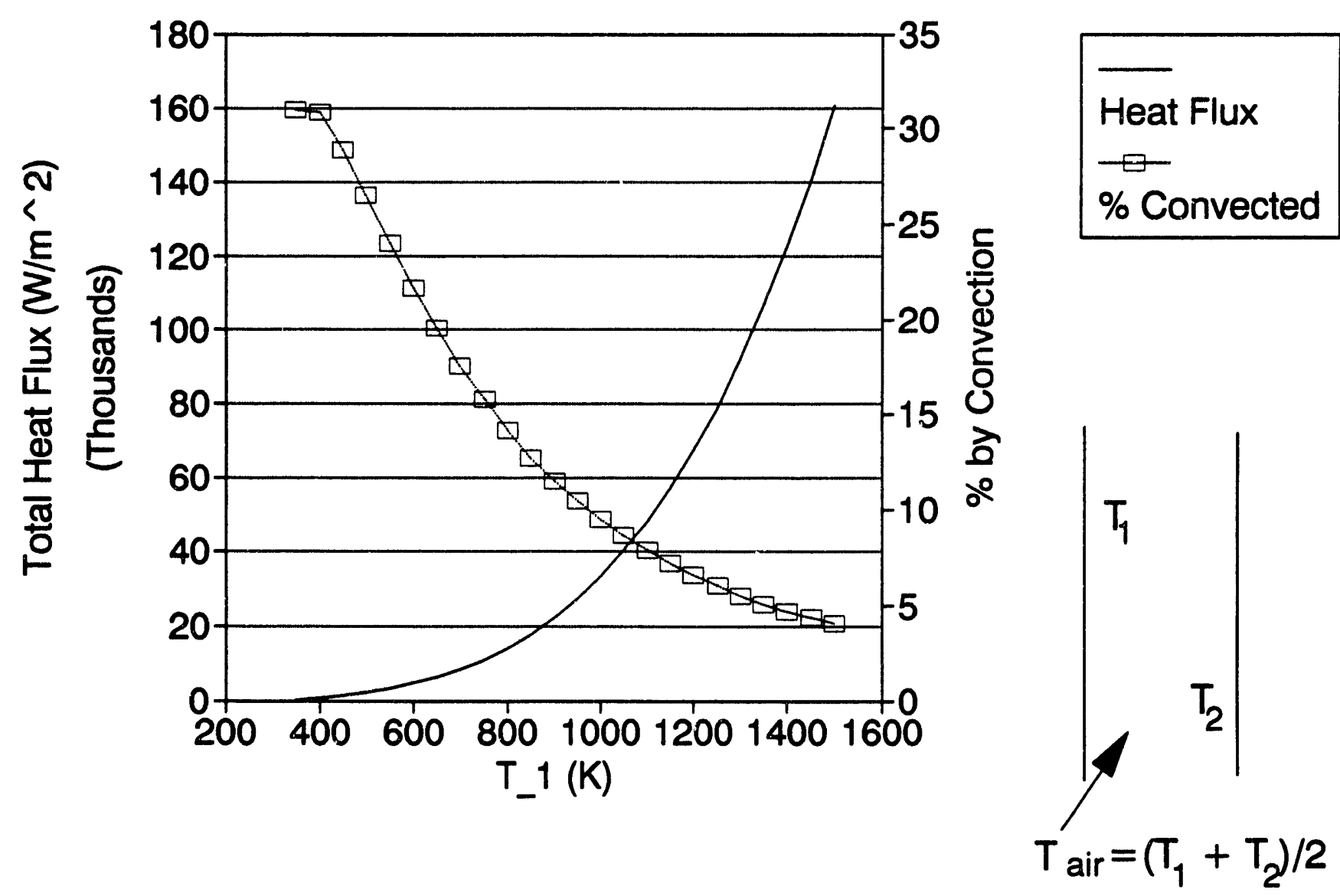

Figure 7: Combined radiation and convection heat flux between planes. 
$P_{i j}=\frac{\delta_{i j}}{1-\epsilon_{i}}-F_{i j}$ when $\epsilon_{i}<1$ or $P_{i j}=\delta_{i j}$ for black surfaces. ${ }^{5} b_{i}=\frac{\epsilon_{i}}{1-\epsilon_{i}} E_{i}$ for $\epsilon_{i}<1$ and $b_{i}=E_{i}$ for black surfaces. The quantity, $[P]^{-1} \bar{b}$, gives the vector of radiosities. ${ }^{6}$

Equation (18) is a very compact statement of the radiating enclosure analysis. In the current case we follow all the surface temperatures through the simulation and this statement is precisely what is needed to evaluate the $q_{i}$ of the system which let us evaluate the instantaneous rates of change of surface temperatures. Eqs. (11) and (13) are typical of those that are integrated to track interior surface temperatures. The temperatures of surfaces $A_{4}$ and $A_{6}$ are modified only by $q_{4}$ and $q_{6}$ as their backsides are regarded as being perfectly insulated.

\subsubsection{View Factor Estimation}

In multidimensional analyses, a matter of considerable complexity is the evaluation of the $F_{i j}$, the view factors, for the enclosure surfaces. When these are derived by direct integration (Eq. (1)) the task gets unwieldy very fast. In the current analysis approximate view factors which obey reciprocity and conservation considerations are developed.

In the present model the location of the surface area associated with inert cargo, $A_{3}$, is only vaguely defined. $A_{3}$ is dispersed throughout the volume confined by $A_{4 . .9}$. This feature of MELTER is motivated by the need for the current modeling effort to be flexible enough to accommodate a wide range of payloads. transm and ttrans are code inputs used to specify average transmittances for enclosure surfaces viewing across the enclosure and for the cargo viewing an enclosure wall. Both of these transmittances have a value between 0 and 1 corresponding, respectively, to unobstructed and totally obstructed views. ttrans=1 has generally been used, which corresponds to the cargo having a clear view of SST interior walls. transm is assigned on a cargo by cargo basis. If the cargo fills the SST so that there are few unobstructed views across the SST transm tends towards zero. Conversely, if the cargo is best represented as an isolated entity on the SST's centerline it will generally not obstruct radiant exchange across the enclosure and the value of transm will tend towards unity. In the limit of transm=0 none of $A_{4 . .9}$ can exchange energy directly with each other. In the other extreme, $\operatorname{transm}=1$ and ttrans $=1, A_{3}$ can not exchange energy with any other surface in the enclosure. The procedures used are as follows:

1. The surface area of the cargo, acargo, is partitioned into $A_{1}$ and $A_{2}$ such that

\footnotetext{
${ }^{5} \delta_{i j}=1$ if $i=j$ and zero otherwise.

${ }^{6}$ The radiosity of a surface, mentioned in any introductory treatment of radiation heat transfer, is the sum of all radiant energy leaving a surface. That is, the reflected and emitted energy. For black surfaces the radiosity and emissive power, $E$, of the surface are the same. Conversely, for highly reflecting enclosures, surfaces may have a radiosity that is much greater than $E$.
} 
$A_{1} / A_{2}=\sum A_{5,7 . .9} / \sum A_{4,6}$. That is, $A_{1}$ is to the whole of the cargo's exterior as the area of the directly heated exterior of the SST is to its whole exterior area.

2. The view factors between the six interior surfaces of the SST $\left(A_{4.9}\right)$ are estimated as though the box is empty.

(a) A variable $A^{*}$ is set which is considered to be the largest area a surface can have without seeing itself. $A^{*}$ is equivalent to a side of the SST.

(b) $F_{i, j}=A_{j} /\left(A_{t o t}-A_{i}\right)$ and $F_{i, i}=0$ if $A_{i}<A^{*}$ otherwise $F_{i, j}=A_{j} /\left(A_{t o t}-A^{*}\right)$ and $F_{i, i}=\left(A_{i}-A^{*}\right) /\left(A_{t o t}-A^{*}\right)$ where $A_{\text {tot }}$ is the whole interior area of the SST.

(c) The view factors from each area to all others are calculated in ascending order of size. To describe this process let us use subscripts a..f to correspond to the interior surface areas in order of size ( $a$ is smallest). Conservation and reciprocity are applied along the way. That is:

i. $F_{a, i}$ are calculated for $i=a . . e$ and then $F_{a, f}=1-\sum_{i=a}^{e} F_{a, i}$

ii. $F_{b, a}=A_{a} F_{a, b} / A_{b}, F_{b, b . . e}$ are found according the rules of $2 \mathrm{~b} . F_{b, f}=$ $1-\sum_{i=a}^{e} F_{b, i}$

iii. $F_{c, a}=A_{a} F_{a, c} / A_{c}, F_{c, b}=A_{b} F_{b, c} / A_{c}$, etc...

The benefit of calculating the view factors for areas in ascending order of size is that reciprocity is always applied so that the area ratio used is less than 1. If the reciprocity rule is applied in the other order between a small surface and a large surface, the impossible circumstance of a view factor exceeding unity can be predicted. After this process is completed, the view factors associated with $A_{a . . f}$ are reordered to map onto $A_{4 . .9}$ as defined in Figure 1. Each of these viewfactors (between interior wall areas) are then attenuated by a transmittance specified in code input transm. The amounts by which the view factors diminish during this process are recovered later in view factors between these surfaces and $A_{3}$, the inert cargo. In the limit of transm approaching zero each of the interior surfaces $\left(A_{4 . .9}\right)$ would have a unit view factor to the inert cargo.

3. $F_{i, j}$ for $A_{1}$ and $A_{2}$ are zero to themselves and each other.

4. A variable proximity, input to the code, is used to indicate whether the cargo is isolated on the SST's centerline (proximity $=0$ ) and away from the walls or is positioned close to the wall (proximity=1). The view factors from $A_{1}$ to the heated surfaces $A_{9}, A_{8}, A_{7}$, and $A_{5}$ are calculated in that order.

(a) If proximity=0 $F_{1, i}=A_{i} / A_{5,7.9} \times$ ttrans. 
(b) If proximity=1: $F_{1,9}=\min \left(A_{1}, A_{9}\right) / A_{1} \times$ ttrans; $F_{1,8}$ is the minimum of $\min \left(A_{1}, A_{8}\right) / A_{1} \times \operatorname{ttrans}$ and $1-F_{1,9} ; F_{1,7}$ is the minimum of $\min \left(A_{1}, A_{7}\right) / A_{1} \times$ ttrans and $1-F_{1,8}-F_{1,9}$; and $F_{1,5}$ is the minimum of $\min \left(A_{1}, A_{5}\right) / A_{1} \times$ ttrans and $1-F_{1,7}-F_{1,8}-F_{1,9}$. This approach gives $A_{1}$, and hence the threatened cargo, preferential access to the most severe of $A_{5,7.9}$.

For values of proximity between 0 and 1 the preceding rules are mixed in linear proportion.

5. $F_{2,4}=A_{4} /\left(A_{4}+A_{6}\right)$ and $F_{2,6}=A_{6} /\left(A_{4}+A_{6}\right)$.

6. View factors between $A_{3}$ and other areas are calculated by conservation and reciprocity. For example, $F_{2,3}=1-\sum_{i \neq 3} F_{2, i}$ and $F_{3,2}=F_{2,3} A_{2} / A_{3}$.

The preceding steps are completed even when some of $A_{4 . .9}$ are equal to zero. ${ }^{7} \mathrm{Be}$ fore the radiation matrix equations are conditioned, zero-area $A_{i}$ 's are removed from consideration.

\subsection{Cargo Model}

Before MELTER formulates the model for a given scenario the cargo is known to it as a series of spherically concentric shells. The shells have thermal capacitance $\left(M C_{p}\right)_{i}, i=$ 1, ncs, where ncs is the number of spherical annuli representing the cargo. The radiation enclosure view factor calculations are described in the previous section, but the first part of that analysis divides the whole surface area of the cargo into $A_{1}$ and $A_{2}$. The variable split (also referred to as $\beta$ later) is the $A_{1}$ 's fraction of the whole surface area of the cargo. Figure 8 is a schematic rendering of the cargo as it is modeled. Shown is a model for which ncs $=5$. The interior annulus is node 1 and the nodes to the left (towards the hot surface) are nodes $2,4,6, \& 8$. The nodes to the right of center are $3,5,7, \& 9$.

To create the parameters supporting the idealized spherical model of the cargo in question the "shortest path" into the critical portion of the device is chosen as the representative series of thermal barriers from the outside to the inside. This introduces immense conservatism for some cases and at least some conservatism for even the most unfavorable conditions. This is because no matter what orientation the package bears to its most intensely heated surface, there will always be greater thermal capacitance than is being represented to "share the load." Geometries and material specifications supporting this activity for specific cargos are described elsewhere.

\footnotetext{
${ }^{7} F_{i, j}$ is actually carried along during the estimation process as the quantity $A_{i} F_{i, j}$ until after the zero-area surfaces have been dismissed avoiding reciprocity and the divide-by-zero error it would create.
} 


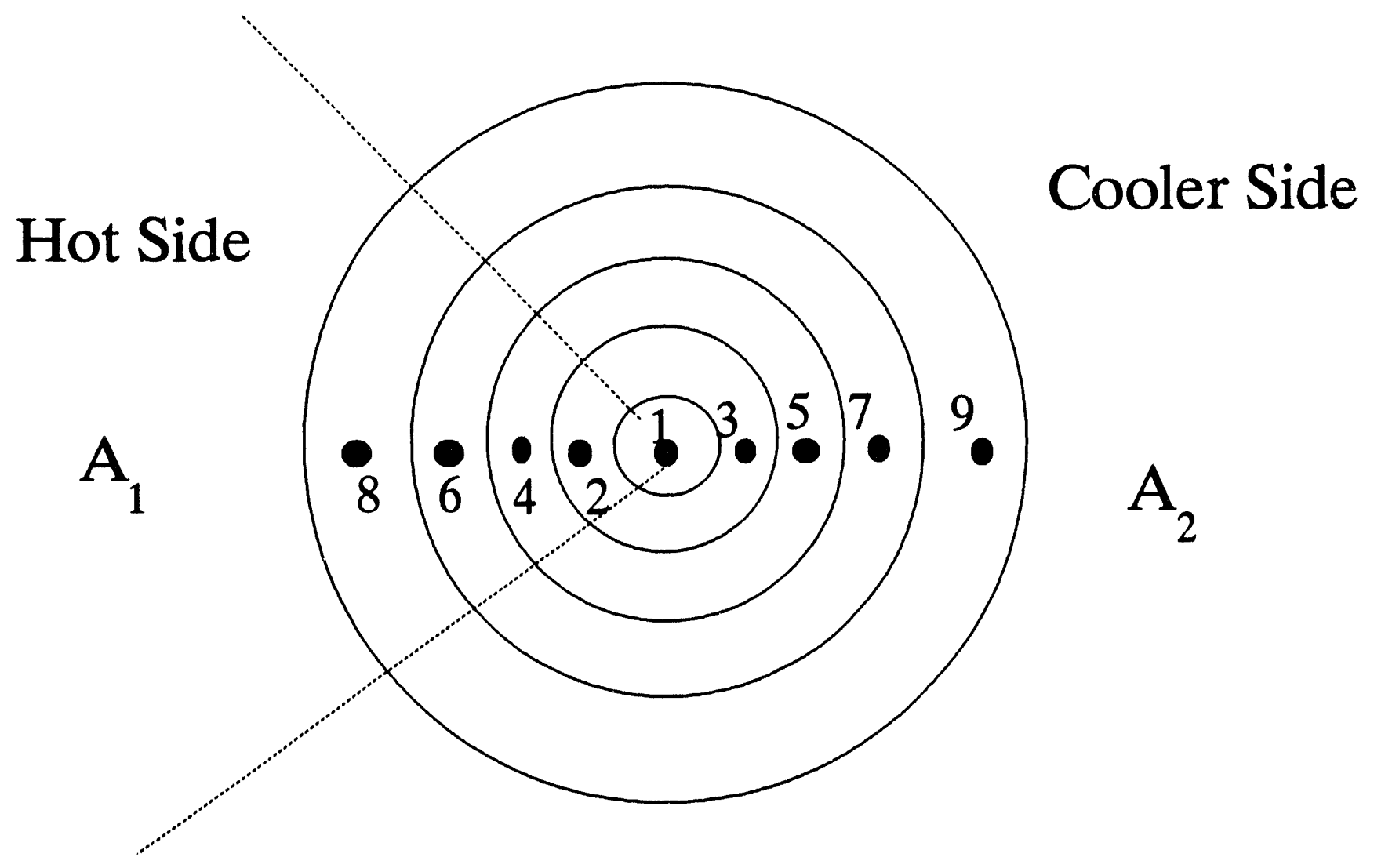

Figure 8: Schematic representation of the cargo model for which ncs=5. 
When the short path is examined its thermal capacitance is concentrated into shells. Concentric shells are "connected" by thermal diffusion and radiative conductors. Thermal diffusion conductors have the value:

$$
\eta_{i}=\frac{4 \pi k}{1 / r_{i}-1 / r_{i+1}}
$$

Radiative conductors have the value:

$$
\omega_{i}=4 \sigma \pi r_{i}^{2}\left[\frac{1-\epsilon_{i}}{\epsilon_{i}}+\frac{1-\epsilon_{i+1}}{\epsilon_{i+1}} \frac{r_{i}^{2}}{r_{i+1}^{2}}+1.0\right]^{-1}
$$

The origins of Eqs. (19) and (20) are explained clearly in Ref. [8]. Their use for a generous number of layers would constitute a rather traditional finite difference model. In the present model the number of concentric shells used is minimized for computational expediency. As a result, the model suggested by Equations (19) and (20) is first used with sufficiently fine discretization to yield reasonable results as the cargo is subjected to reference boundary conditions. The resulting solution is then used as a reference to which a coarser model is fit. In the coarser model the $\eta_{i}$ of Eq. (19) are replaced by values that optimize the agreement between the fine and coarse models. This procedure is documented elsewhere.

Given the $\omega_{i}$ and $\eta_{i}$, the heat transfer to a spherical shell sandwiched between two shells for an entire sphere would be:

$$
Q_{i}=\left(T_{i+1}^{4}-T_{i}^{4}\right) \omega_{i}+\left(T_{i-1}^{4}-T_{i}^{4}\right) \omega_{i-1}+\eta_{i}\left(T_{i+1}-T_{i}\right)+\eta_{i-1}\left(T_{i-1}-T_{i}\right)
$$

The preceding equation is written as if the sphere is strictly the one-dimensional geometry after which the conductors are fashioned. In the present model the situation is complicated by the splitting of the exterior surface of the cargo into $A_{1}$ and $A_{2}$. We have a pseudo-spherically-symmetric-1-D analysis. The conductors of Eqs. (19) and (20) are apportioned to the hot and cool sides of the cargo. For example, for the case in which ncs $=5$ the equations that are integrated are:

$$
\frac{\partial T_{1}}{\partial t}=\left\{\beta\left[\left(T_{2}-T_{1}\right) \eta_{1}+\left(T_{2}^{4}-T_{1}^{4}\right) \omega_{1}\right]+(1-\beta)\left[\left(T_{3}-T_{1}\right) \eta_{1}+\left(T_{3}^{4}-T_{1}^{4}\right) \omega_{1}\right]\right\} /\left(m C_{p}\right)_{1}
$$

In Eq. (22) $\beta$ (the same as split in the code) represents the fraction of the sphere "belonging" to the hot side of the sphere. $m C_{p}$ is the thermal capacitance of the entire spherical layer. As an example of an interior node:

$$
\frac{\partial T_{2}}{\partial t}=\left\{\left(T_{4}-T_{2}\right) \eta_{2}+\left(T_{4}^{4}-T_{2}^{4}\right) \omega_{2}+\left(T_{1}-T_{2}\right) \eta_{1}+\left(T_{1}^{4}-T_{2}^{4}\right) \omega_{1}\right\} /\left(m C_{p}\right)_{2}
$$

Eq. (23) is typical of equations for either the hot or cool sides. The factors $\beta$ and $(1-\beta)$ don't appear because they modify the net heat transfer rate and the thermal 
capacitance of the node in like proportion (they cancel out). For the exterior node (node 8 for the hot side in this case) the sphere communicates thermally with the enclosure analysis. The resulting equation is:

$$
\frac{\partial T_{8}}{\partial t}=\left\{\beta\left[\left(T_{6}-T_{8}\right) \eta_{4}+\left(T_{6}^{4}-T_{8}^{4}\right) \omega_{4}\right]+q_{1} A_{1}\right\} /\left(m C_{p}\right)_{5} / \beta
$$

where $q_{1} A_{1}$ comes from the radiating enclosure analysis (See Eq. (18)). In Eqs. (2224 ), note that the $T_{i}$ are described as in Figure 8 so as you proceed radially outward the subscript on $\omega$ and $\eta$ only grows half as fast as that on $T$ (there are two $T$ 's per shell but only one new $\omega$ or $\eta$ ).

$\omega$ and $\eta$, as described in the preceding paragraphs are consistent with finite difference theory and should provide an accurate one-dimensional model if sufficiently many layers are used. In the present model we wish to use a modest number of layers. The result of this coarseness will generally be a model that responds too quickly. However, if the foregoing theory is applied, care must be taken so that the critical temperature to be monitored is not made part of a thick material region. Since each "node" is (spatially) isothermal, not resolving the gradient of temperature through a region can result in its temperature being suppressed.

Variations of Eq. (24) are used to simulate melting of the exterior shell of the cargo on the hot side. Eq. (24) is integrated as it is written while $T_{8}$ heats up to tmelt. Once $T_{8}$ reaches tmelt, the meaning of $T_{8}$ changes. Eq. (24) continues to be integrated but tmelt is substituted for $T_{8}$ on the right hand side of the equation. Likewise, the constant temperature tmelt is used in the radiation enclosure analysis during this interval. During the melting the variable $T_{8}$ actually represents tmelt $+\int_{0}^{t} q\left(t^{*}\right) /\left(C_{p} \rho h\right) d t^{*}$ until the integrated heat addition accounts for melting through the thickness. umelt, a code input, is the melting material's heat of fusion divided by the its constant value of specific heat. The melting is complete when $T_{8}=$ tmelt + umelt.

After the melting is complete, the temperature of the next spherical shell in is substituted for the temperature of $A_{1}$ in the enclosure analysis. The optio-geometric properties of the SST are not recomputed. Instead, the system is allowed to depart slightly from conserving energy. The energy $q_{1} A_{1}$ from the enclosure analysis is reduced by the factor $\left(r_{6} / r_{8}\right)^{2}$ as it is applied to the node corresponding to $T_{6}$. Of course, the subscripts 6 and 8 used in the preceding sentence are only correct for ncs $=5$. No adjustments are made to $\epsilon_{1}$ during this process.

To effect damaged cargo, four damage states, designated 0 through 3 can be communicated to MELTER (See the input descriptions in Secs. 4.3.1 and 4.3.2.). Damage state 0 corresponds to intact cargo. Damage states $1,2, \& 3$ correspond to cargo models with increasingly many layers stripped away. Damage state 3 always corresponds to the critical layer being exposed. The number of layers constituting models corresponding to the intermediate damage states are known to MELTER through information it reads 
in from cargo.TAR (See Section 4.3.2).

\subsection{Failure Criteria}

One layer of the foregoing cargo model is designated as the sensitive layer. The temperature of that layer is monitored to determine if failure has occurred. MELTER accepts as input a user-specified temperature-time curve ${ }^{8}$ which defines the temperature at which the sensitive layer fails as a function of time. Figure 9 shows qualitatively such a temperature-time failure criterion. In MELTER conservatism is introduced in that "time zero" is taken to be the start of the fire. Depending upon the scenario, there may be a very significant delay between the start of the fire and the onset of appreciable heating to the cargo. No credit is taken for that delay which may be considerable for some scenarios. Curves A, B, C, and D represent four different temperature histories that might result at the sensitive layer in order of decreasing scenario severity. Failures associated with Curves A, B, and C are assumed when the curve intersects the failure curve. Curve $D$ suggests no failure. MELTER monitors the response of the sensitive layer and the failure curve. For scenario D MELTER will conclude that no failure occurs at the time designated $t_{\text {safe }}$. The conditions allowing the "safe" conclusion that the curves never will intersect are that fire is extinguished and that $\frac{\partial T_{\text {crut }}}{\partial t}<\frac{\partial T_{f c}}{\partial t}$. That is, the time rate of change of the critical layer must be more negative than that corresponding to the failure curve. Note that for Curve $\mathrm{C}$ the failure occurs at a time after the maximum value of $T_{\text {crit }}$ is realized.

\subsection{Numerical Methods}

The numerical methods used to render results from the foregoing equations are fairly trivial. Three significant requirements arise:

1. Integration: A method of integrating the 1st-order coupled differential equations that have been developed must be incorporated. MELTER was developed using a Runge-Kutta routine (Ref. [9]). Subsequently, ode (Ref. [10]), a more sophisticated integrator, has been implemented for greater efficiency.

2. Matrix Inversion: The radiation problem requires a matrix to be inverted. That matrix is at most $9 \times 9$ in size and is always well-behaved and easily inverted. A simple Gauss-Jordan method with pivoting is employed.

3. Root-Finding: When MELTER is to find the minimum fire duration that leads to failure a root-finding method is needed. Bisection is used. Generally speaking,

${ }^{8}$ This curve is defined in a piecewise linear fashion with parameters defined in the input file defining the cargo. See Section 4.3.2. 


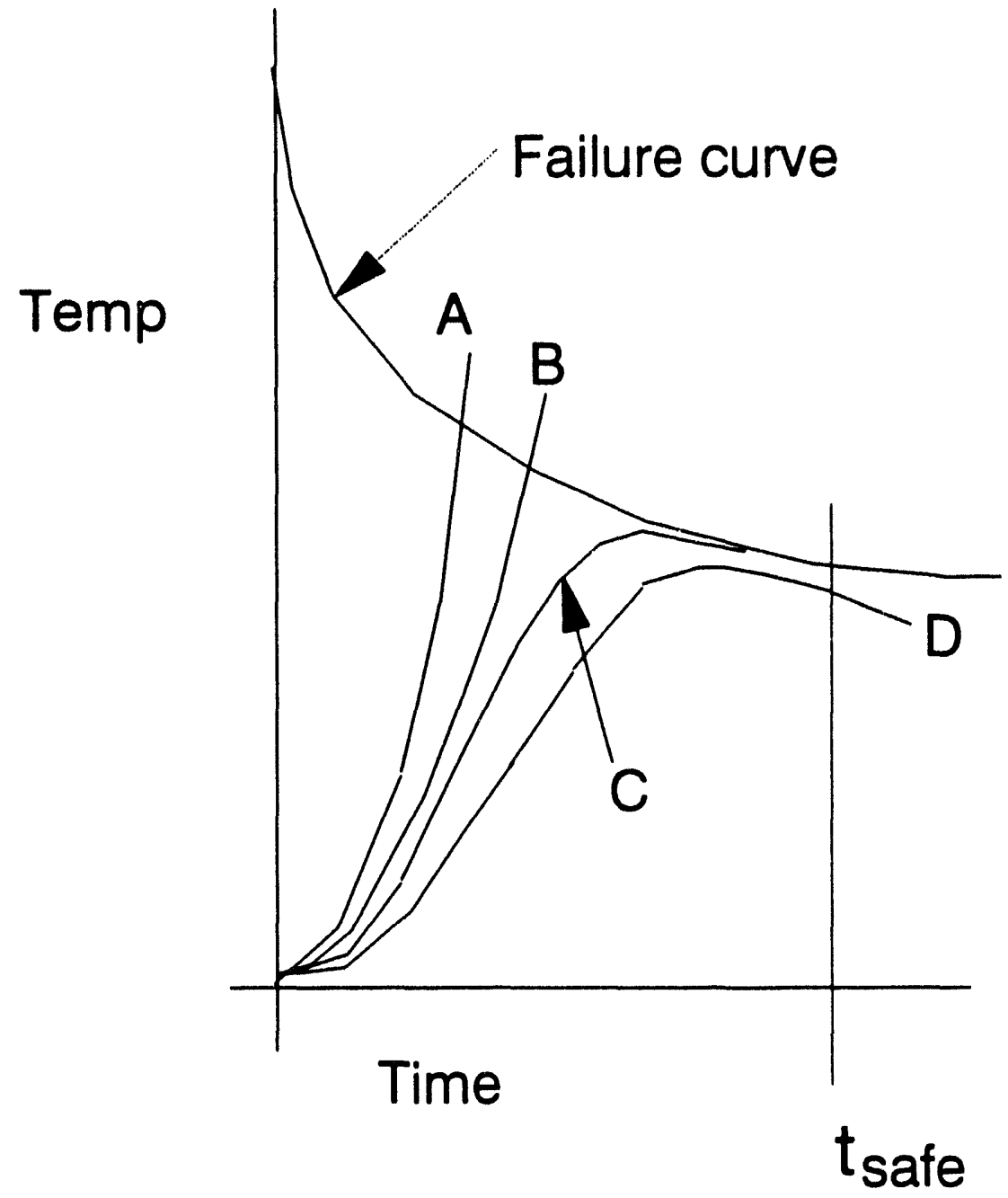

Figure 9: Temperature-time failure criteria used in MELTER and four hypothetical cargo responses. 
bisection is unsophisticated and not famous for speed. However, in the present application it is effective. Bisection requires that upper and lower bounds be known for the search variable. A very good estimate is found for the upper bound by "leaving the fire on" until a failure occurs. Zero fire duration makes a good lower bound which will only ever be approached by halving the uncertainty interval "from the right" so that it is efficiently replaced with a more appropriate lower bound. Finally, the present implementation is efficient because the integration never needs to begin earlier than the current lower bound on the search variable. That is, the state of the system is saved at the time defining the lower end of the uncertainty interval. In subsequent search steps the integration can always be resumed at that point. This aspect of MELTER works very well. 


\begin{tabular}{||l|l|r|r|r|r||}
\hline \hline & Carameter & Case & Case & Case & Case \\
& II & III & IV \\
\hline \multirow{5}{*}{ Inputs: } & Fire Temperature $(K)$ & 2133 & 2133 & 2133 & 2133 \\
& Fire Diameter $(m)$ & 9.1 & 9.1 & 9.1 & 9.1 \\
& Fire Displacement $(m)$ & 0 & 5.6 & 5.6 & 11.7 \\
& Fire Duration $(s)$ & 1800 & 2400 & 2400.0 & 2400.0 \\
& $A_{8},\left(m^{2}\right)$ & 0.0 & 0.9 & 0.0 & 0.0 \\
& $A_{9},\left(m^{2}\right)$ & 0.0 & 0.9 & 5.6 & 0.0 \\
\hline & $F_{A_{7}, f}$ & 1.0 & 1.0 & 1.0 & 0.3 \\
& $A_{1},\left(m^{2}\right)$ & 6.2 & 2.0 & 2.0 & 2.5 \\
& $A_{3},\left(m^{2}\right)$ & 200.0 & 200.0 & 200.0 & 200.0 \\
Computed & $A_{4},\left(m^{2}\right)$ & 2.3 & 20.4 & 20.4 & 20.4 \\
Quantities: & $A_{5},\left(m^{2}\right)$ & 18.1 & 0.0 & 0.0 & 0.0 \\
& $A_{6},\left(m^{2}\right)$ & 19.7 & 47.7 & 47.7 & 42.4 \\
& $A_{7},\left(m^{2}\right)$ & 49.5 & 19.6 & 16.9 & 26.8 \\
\hline Results: & Time to failure, $(s)$ & 2230 & 1630 & 620 & none \\
& $T_{\text {crit }}$ at failure, $(K)$ & 519 & 535 & 570 & \\
\hline \hline
\end{tabular}

Table 1: Summary of cases considered.

\section{Case Studies}

As it will be used in the risk assessment, MELTER determines the minimum fire duration which, for other conditions specified, leads to a failure. MELTER also can be operated with a user-specified fire duration. In that case the transient thermal response of the SST is calculated from zero time until a failure occurs or until it can be asserted that no failure will occur. Table 1 summarizes four case studies that are included to demonstrate the qualitative nature of the SST's response for several scenarios of interest. Figure 10 represents the geometry of all four cases as an aid to visualize the relative size of the SST, fire, and separation distance. All of these fires feature a $2100 \mathrm{~F}$ fire.

\subsection{Case I}

The first case considered is one for which the fire is centered under the SST and is of sufficient size to engulf most of the SST's exterior. Table 1 gives the quantitative distribution of $A_{1 . .9}$ associated with the fire. For Case I, the SST is in nominal condition at the start of the fire $\left(A_{8}=A_{9}=0\right)$. Referring to Figure 11, several temperatures of 


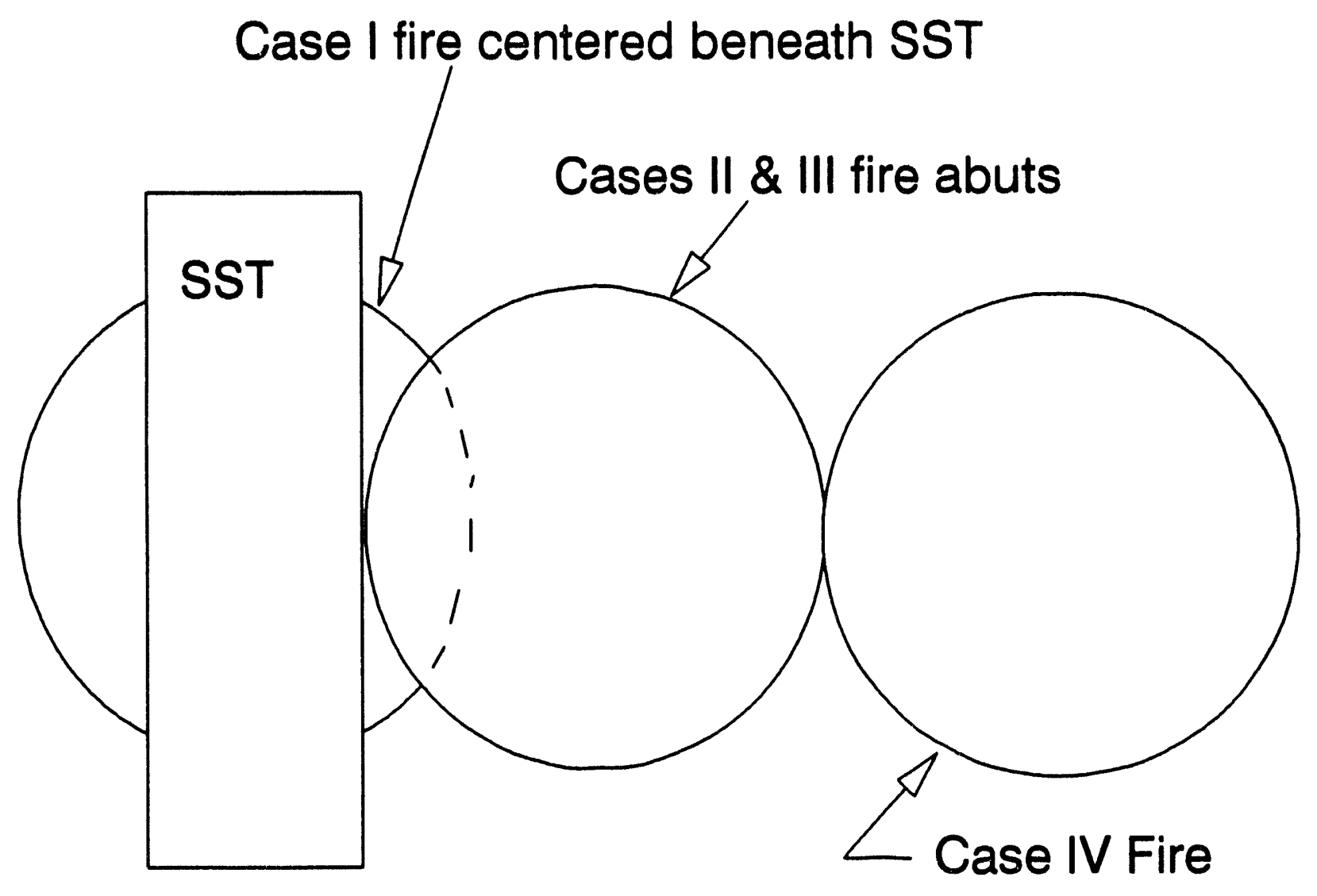

Figure 10: Schematic representation of geometries for four case studies. 
interest and the char displacement $(x)$ in the wall are plotted.

- The analysis stops at $2230 \mathrm{~s}$, which is more than $400 \mathrm{~s}$ after the fire is extinguished. The critical state is reached after the fire's extinction due to the energy that was stored in the SST's walls. The cargo used in this example has a relatively thin outer layer coupled to the next layer in by a radiation gap.

- $T_{\text {crit }}$ at failure is $519 K$.

- The directly heated armor $\left(A_{7}\right)$ and case temperatures outrun the temperature associated with secondary heating $\left(A_{6}\right) .^{9}$

- At this high temperature and unit view factor, the foam in the wall chars rapidly, introducing only about a $100 s$ delay before heat is "felt" on the interior.

- The thin outer skin rapidly moves to a quasi-equilibrium temperature very close to the fire's temperature after the start of the fire.

\subsection{Case II}

In Case II the fire is the same size but it is displaced so that the near edge of the fire just coincides with the fireside edge of the SST. Note in Table 1 how $A_{1 . .9}$ are redistributed. In this case nearly a square meter $\left(10 f t^{2}\right)$ of $A_{8}$ and $A_{9}$ have been specified. $A_{8}$ corresponds to damage to the SST which is associated with removal of the exterior skin and foam down to the armor. $A_{9}$ corresponds to a complete breach of the SST. $A_{9}$ and $A_{8}$ are both in line with the fire. Referring to Figure 12:

- For Case II the early SST skin temperature history and char displacement are identical to the first case since the view factor to the fire is still unity and the fire temperature is unchanged.

- The temperature associated with bare armor $\left(A_{8}\right)$ rapidly moves to quasi-equilibrium. This temperature is lower than the skin temperature of the previous case because the heat flux through the bare armor is higher than that through the charred wall.

- The cargo case temperature outruns the armor temperature associated with $A_{7}$ because of its clean view to the fire through $A_{9}$ and to the directly heated armor $\left(A_{8}\right)$.

\footnotetext{
${ }^{9}$ Referring to the conservative rules that are used for determining view factors, the "sunny" side of the cargo is generally given a clean view of the hot armor.
} 


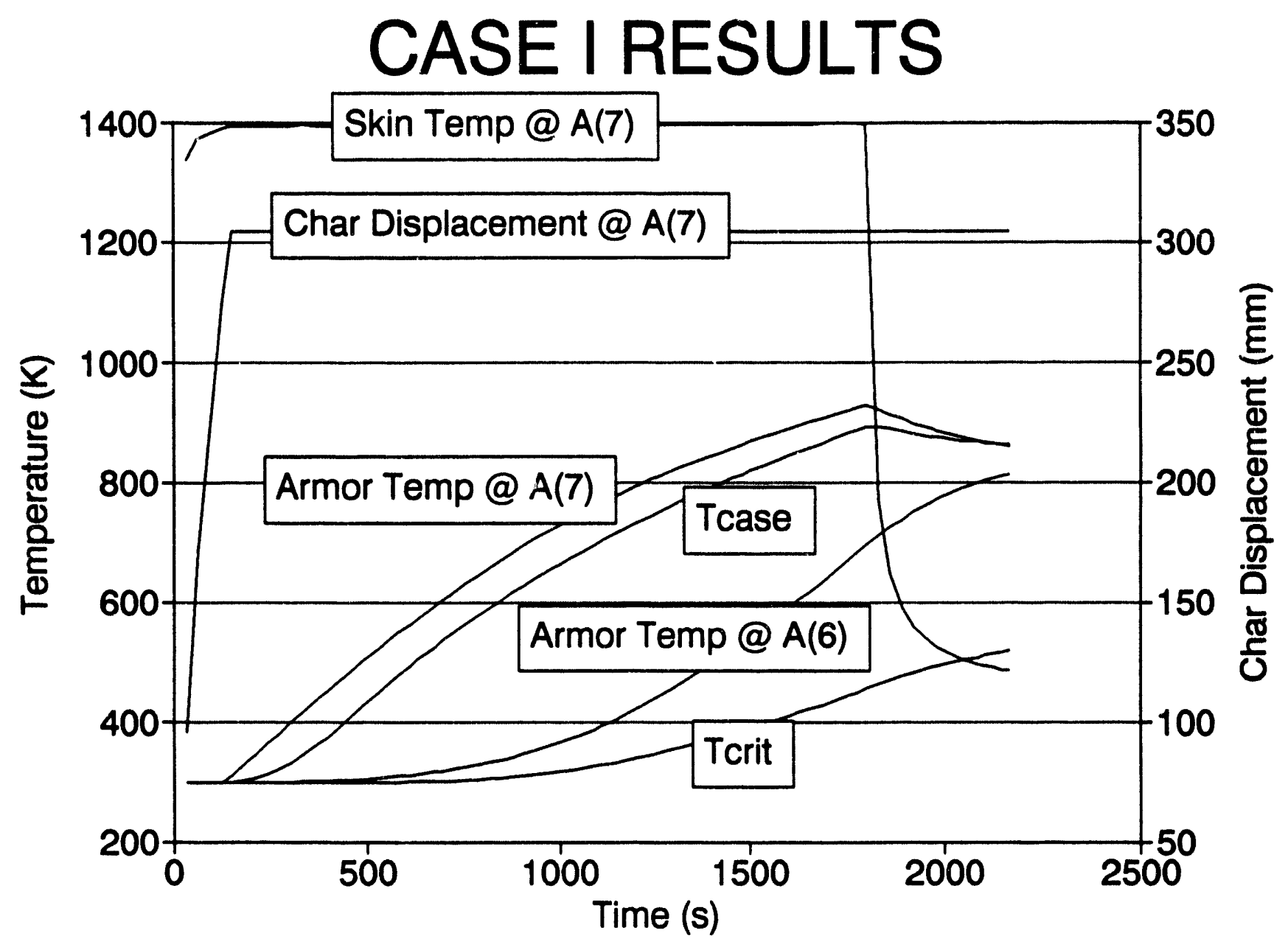

Figure 11: Case I results. 
- $T_{\text {crit }}$ at failure is $535 \mathrm{~K}$. The heating rate for this case is greater than that for the first case and a higher failure temperature is used.

\subsection{Case III}

The fire geometry for Case III is identical to the previous case. A very significant hole $\left(5.6 \mathrm{~m}^{2}, 50 \mathrm{ft}^{2}\right)$ has been opened on the side of the SST. Referring to Figure 13:

- The case temperature climbs very rapidly to its melting point (933K) and is flat during the melting process which is not completed at failure.

- The failure time is shortened to $620 \mathrm{~s}$ and a corresponding increase of $T_{\text {crit }}$ to $570 K$ is observed.

\subsection{Case IV}

Case IV corresponds to a scenario for which there is no structural damage to the SST and there is considerable separation distance between the fire and the SST. Referring to Table 1 notice that $A_{7}$ is larger than for the previous case which may be counterintuitive to the reader. However, referring to Eq. (6) appreciate that $E_{\max }$ is decreased for this case (to about $30 \%$ of $E_{f}$ ) so that the energy deposition for this case is appropriately less than that for the previous case. As a result of the separation distance this fire is not particularly threatening. The fire duration is an hour for this case and no failure occurs. Referring to Figure 14:

- The skin temperature again moves quickly to a quasi-equilibrium temperature of about $1040 \mathrm{~K}$ which roughly corresponds to an emissive power that is about $30 \%$ of that of the fire (see Eq. (4)).

- The charring of the wall takes about $540 s$ or about 5 times as long as the previous cases for this reduced heating rate.

- The cargo's case temperature does not lag far behind the temperature associated with $A_{7}$. Due to the modest heating rate, the whole interior of the SST will be comparatively isothermal.

- The analysis stops at around $4300 s$ when the temperature of the sensitive layer "turns around." This is about $900 s$ after the fire is extinguished. 


\section{CASE II RESULTS}

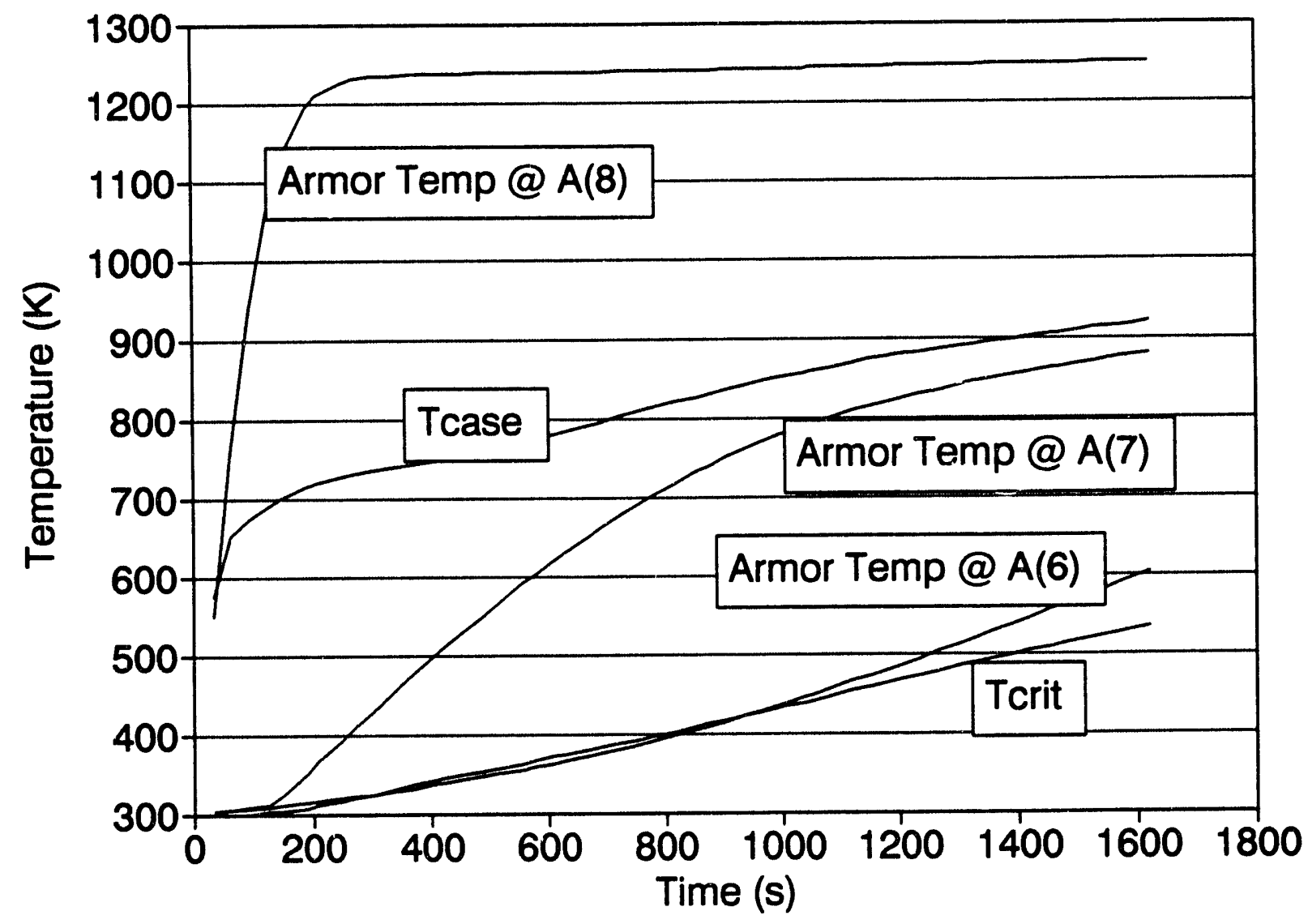

Figure 12: Case II results. 


\section{CASE III RESULTS}

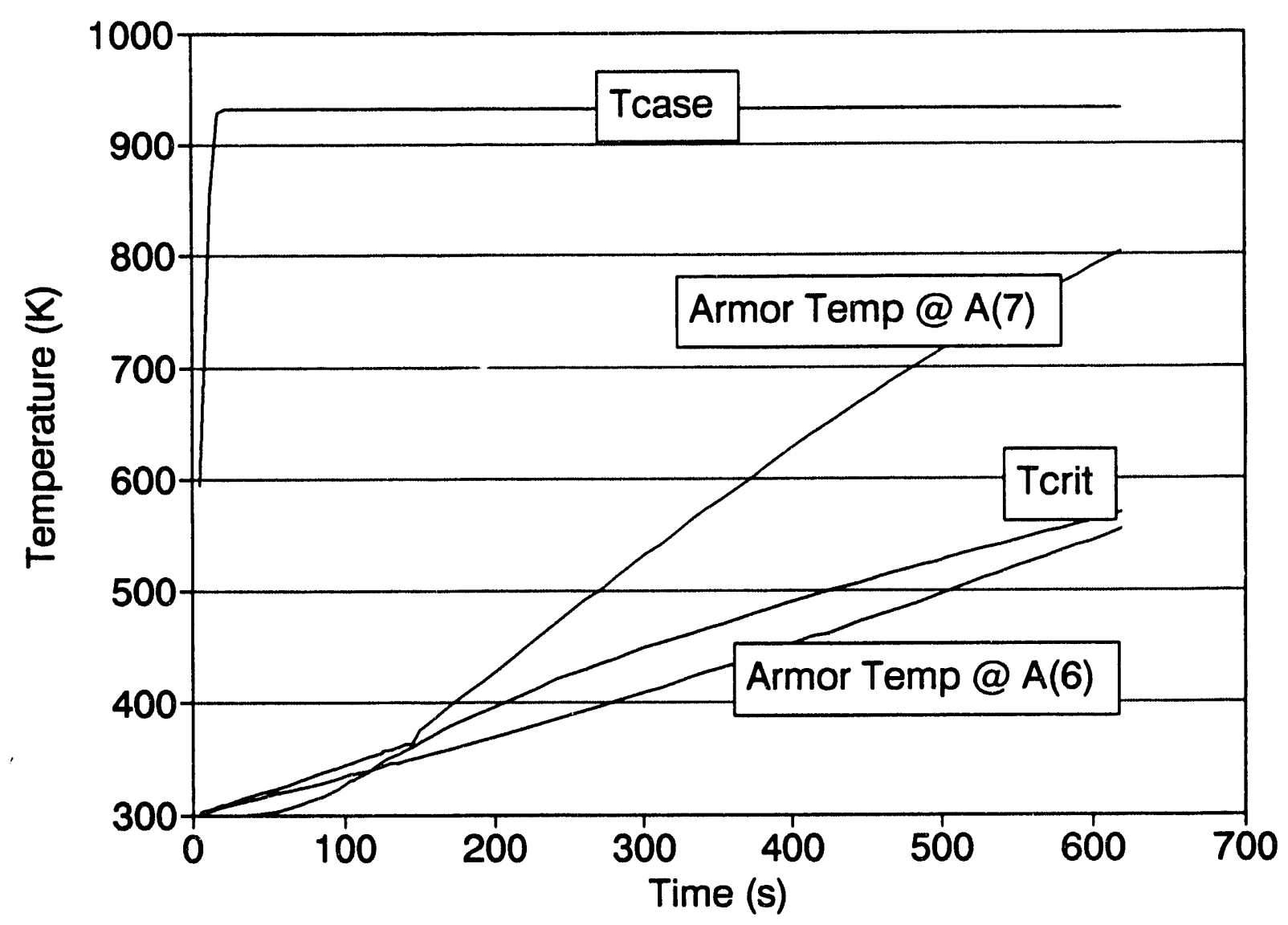

Figure 13: Case III results. 
CASE IV RESULTS

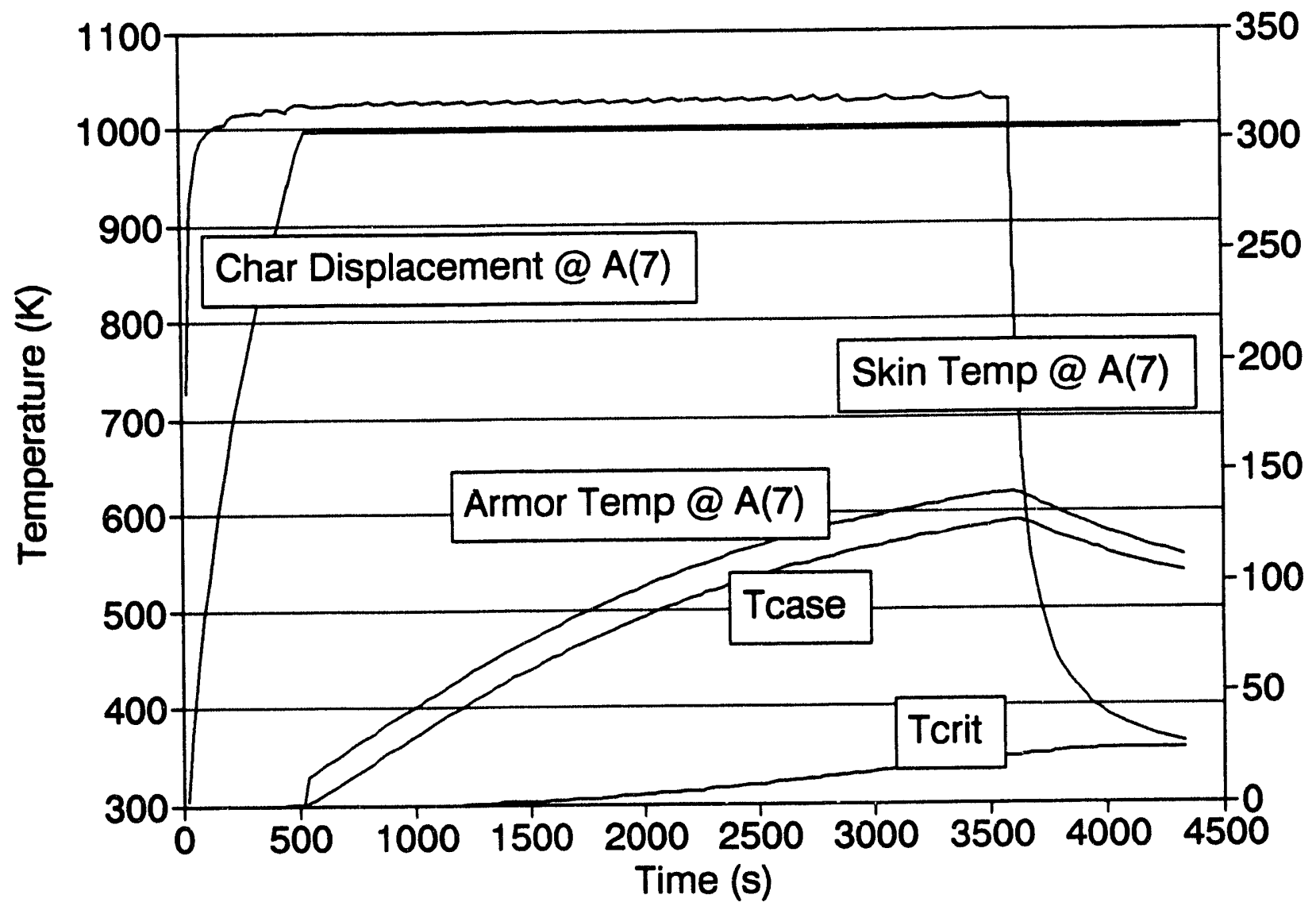

Figure 14: Case IV results. 


\section{MELTER Code Description}

\subsection{Introduction}

This section describes the coding of MELTER, which is written in FORTRAN. As discussed in the first section, information defining an analysis depends on the SST's properties, those of the cargo, and scenario-specific information. Properties of the SST are "hardwired" in the code, that is, the source code must be edited to modify them. The cargo and scenario properties are communicated to the code through interface files.

A listing of MELTER is included in the Appendix $\mathrm{C}$ for reference. The source code for MELTER is about 2500 lines (including ode). Constants defined in the source are all initialized in the BLOCK DATA routine and made available to other subprograms through COMMON statements. MELTER has been operated extensively on SUN SparcStations After compilation, MELTER is executed as follows:

system_prompt: MELTER scenes cargo <carriage return>

scenes and cargo are command line parameters that identify the files containing scenario and cargo information (See Sections 4.3.1 and 4.3.2).

\subsection{Overview of Code Operation}

The steps of the analysis, as they unfold during MELTER's operation, are summarized in the following outline:

1. Read input.

(a) Read scenes. BRC to get scenario-specific parameters.

(b) Read cargo.TAR to get cargo-specific parameters.

2. For each scenario considered construct the model. Most of MELTER's source code serves the purpose of constructing the equations that make up the model.

(a) For a given scenario calculate boundary conditions in terms of exposed area(s) and view factors.

(b) Formulate enclosure radiation problem consistent with the scenario and cargo characteristics.

i. Estimate all view factors needed for enclosure radiation problem. 
ii. Strip out zero area surfaces.

iii. Condition matrix needed for radiation calculations inside SST.

3. Find minimum critical fire duration or whether specified fire duration leads to failure. If minimum critical fire duration is calculated:

(a) Solve equations with fire burning continuously until failure occurs and take that value as the high end of the search interval $\left(t_{\text {high }}\right)$ and $t=0$ as the low end $\left(t_{\text {low }}\right)$.

(b) Reset the fire duration, $t_{d u r}=\left(t_{\text {high }}+t_{\text {low }}\right) / 2$ and restart the thermal simulation.

(c) If failure occurs set $t_{\text {high }}=t_{d u r}$ otherwise set $t_{\text {low }}=t_{d u r}$.

(d) If $t_{\text {high }}-t_{\text {low }}>\min \left(50,0.01 \times t_{d u r}\right)$ go back to (b) otherwise quit.

4. Write results to cargo.MEL.

5. Repeat steps 2-4 for each scenario presently under consideration.

\subsection{Input}

Besides physical characteristics of the SST which are hard-wired into MELTER's source code, inputs are needed to describe the cargo and the scenarios for a particular analysis. MELTER reads problem specification parameters from two input files, cargo.TAR and scenes. BRC. In general, parameters found in cargo.TAR are those that define the cargo and characteristics of the way it is loaded. Parameters found in scenes. BRC are those that define the scenarios to be considered.

\subsection{1 scenes.BRC}

The PRA is facilitated by discretizing the parameter space. That is, discrete values relating to fire size, temperature, and separation; SST damage state reflected through $A_{8}$ and $A_{9}$; and cargo damage conditions reflected through proximity and nstrip are considered. MELTER incorporates loops which exhaustively test every possible combination of discrete values specified for this purpose. Table 2 includes the lines of source code which result in MELTER's reading of scenes. BRC. The variables read are as follows:

nndam number of ndam(i)-prox(i) pairs to be read, a maximum of 4 values may be specified. 
c

open (unit=7,file='branch. dat', status='old')

$c$ there has to be the same number of proximities and

c damage states considered (4 maximum)

$\operatorname{read}(7, *)$ nndam,$(n d a m(i), i=1, n n d a m)$

$\operatorname{read}(7, *) \quad(\operatorname{prox}(i), i=1, \operatorname{nndam})$

$c$ there are the same number of adam9 and adam8 values (6 max)

$\operatorname{read}(7, *)$ nadam $9,(\operatorname{adam} 9(i), i=1, \operatorname{nadam} 9)$

$\operatorname{read}(7, *)(\operatorname{adam} 8(i), i=1, \operatorname{nadam} 9)$

c there may be as many as 10 temperatures

$\operatorname{read}(7, *) n t e m p,(f t e m p(i), i=1, n t e m p)$

$c$ there may be as many as 10 diameters

$\operatorname{read}(7, *)$ ndcdia, $(\operatorname{dcdia}(i), i=1, \operatorname{ndcdia})$

$c \quad$ there may be as many as 7 distances

$\operatorname{read}(7, *)$ ndcdist,$(\operatorname{dcdist}(i), i=1$, ndcdist)

$c$ tduro is zero if the calculation is to yield the minimum critical

c duration. Otherwise it is the specified fire duration.

$\operatorname{read}(7, *) \operatorname{tdur} 0$

close (7)

Table 2: Portion of MELTER that reads scenes. BRC to define scenario parameters. 
ndam (i) an array of integer values (0-3) assigned to nstrip indicating degree of damage to cargo. " 0 " corresponds to undamaged cargo. " 3 " corresponds to the most damaged possible state. Associated information in cargo.TAR tells MELTER the number of layers to peel off of the spherically-idealized model for each value of $\operatorname{ndam}(i)$.

prox (i) (Refer to Section 2.6) an array of values for the variable proximity. These influence the rules used to calculate view factors. For each ndam(i) there must be a corresponding value of prox (i).

nadam 9 number of adam 9 (i)-adam8(i) pairs to be read, a maximum of 6 pairs may be specified.

adam9(i) array of values for the variable $A_{9}$.

adam8(i) array of values for the variable $A_{8}$. There must be one value $c_{-}$adam8(i) for each $\operatorname{adam} 9(i)$.

ntemp the number of $f$ temp ( $i$ ) values to be read up to a maximum of 10 .

ftemp(i) an array of values for fire temperature.

ndcdia the number of dedia(i) values to be read up to a maximum of 10 .

dcdia(i) an array of fire diameters to be considered.

ndcdist the number of dedist(i) values to be read up to a maximum of 7 .

dcdist (i) an array of separation distances to be considered.

tduro specified fire duration. If specified greater than zero the analysis indicates whether that duration leads to failure. If tduro is set equal to zero the minimum critical duration will be determined.

\subsection{2 cargo.TAR}

Table 3 includes the FORTRAN source from MELTER that reads information about the cargo being analyzed subject to all of the foregoing scenario parameters.

ncs 0 the number of concentric shells used to represent the intact cargo. This is the same as ncs for undamaged cargo.

lleft(1) The number of model layers to be included if damage state " 1 " is being considered (see ndam( $i$ ) description in previous section). 


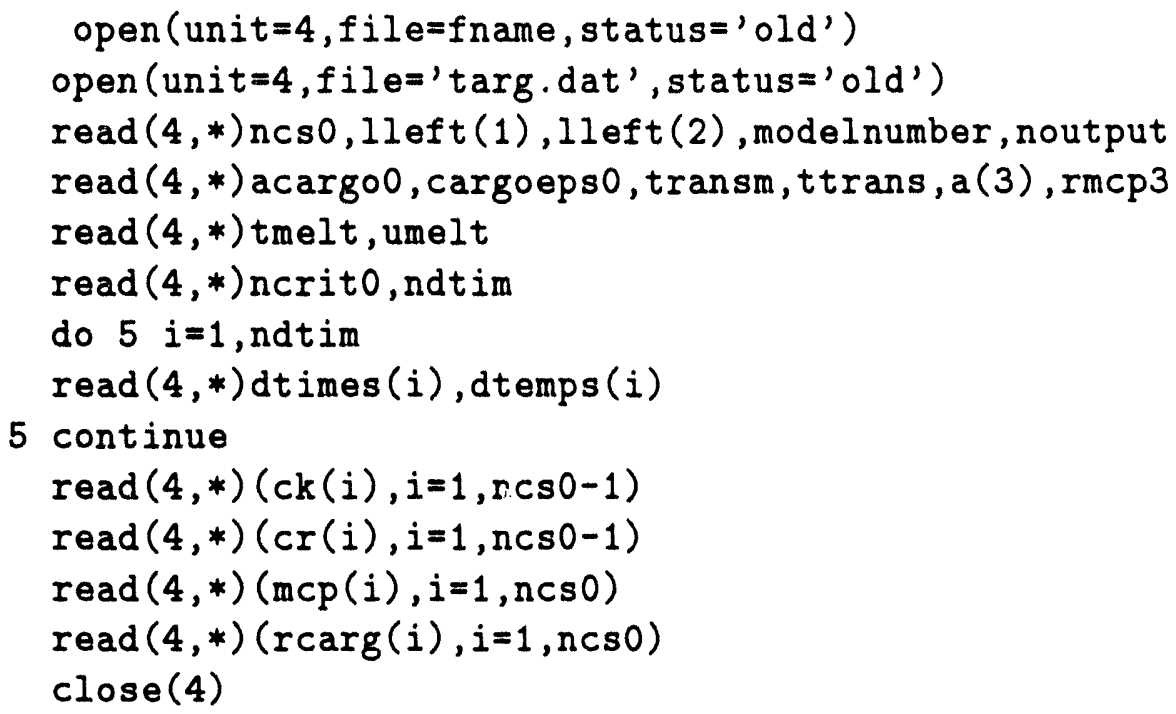

Table 3: Code section that reads cargo. TAR.

lleft(2) The number of model layers to be included if damage state " 2 " is being considered (see ndam(i) description in previous section).

modelnumber An integer used to communicate whether the exterior surface of the cargo is to be allowed to melt. modelnumber $=1$ tells the code that the exterior shell can melt away on the hot side of the cargo.

noutput an integer that is checked to see if the code should generate output. The higher the value, the greater the amount of output generated. Normally, noutput $=0$, in which case the only output of the program is in cargo.MEL.

acargo0 The surface area of the outside of the cargo $\left(\mathrm{m}^{2}\right)$. When many copies of the same cargo item are loaded, this is the exterior area of a single item. The others should be considered as part of the inert cargo.

cargoeps the emissivity of the cargo exterior.

transm an average transmittance for the whole interior. This value should be indicative of the view across the volume. If the SST's volume is packed tightly with cargo this value approaches zero. If the SST is empty or near empty this value approaches unity.

ttrans the transmittance between the threatened cargo and the hottest wall. Usually, we expect this to be higher than the previous value (transin). 
a(3) This area $\left(\mathrm{m}^{2}\right)$ corresponds to the inert cargo. This constant must be specified as some value larger than zero. At present it has no influence on results. ${ }^{10}$

rmcp 3 This is the thermal capacitance $\left(J / K^{*}\right)$ of inert cargo in the SST.

tmelt The melting temperature $(K)$ of the exterior shell of the cargo.

umelt The melting energy of the exterior shell expressed as the latent heat of fusion divided by the constant value of $C_{p}$ that is used to represent the solid during its sensible heating.

ncrito This identifies which concentric shell of the cargo is to be watched for the critical condition. This value must be greater than 1 if a scenario involving damage state 3 is considered.

ndtim This tells how many points are used to describe the failure temperature curve.

dtimes(i) These are ndtim values of time which are used to define the failure temperature curve.

dtemps (i) These are ndtim values of temperature which are used to define the failure temperature curve.

ck(i) These are conduction-style connectors between concentric shells. ck(i) connects $m C p_{i}$ to $m C p_{i+1}$. (see Section 2.7 )

cr(i) These are radiation-style connectors between concentric shells. $\operatorname{cr}(i)$ connects $m C p_{i}$ to $m C p_{i+1}$. $\left(\omega_{i} / \sigma\right.$, Eq. $\left.(20)\right)$

mcp (i) These are the thermal capacitances of concentric shells representing the cargo.

rcarg(i) Radii of shells.

Generally scenes.BRC contains the scenario-dependent parameters and cargo.TAR contains the parameters that describe the package and information about the configuration in which it is shipped in the SST. However, some information must be communicated cooperatively. For example, MELTER allows four levels of cargo damage to be considered which correspond to damage states 0 through 3 . These are specified in scenes.BRC. However, information to interpret the meaning of the damage state must be specified in cargo.TAR for each cargo. As indicated earlier the variables lleft (1)

\footnotetext{
${ }^{10}$ As MELTER is presently formulated rmcp3 gives the total thermal capacitance of the inert cargo, and ttransm specifies that cargo's transmittance. While $a(3)$ is used in the calculations its magnitude has no effect. Its identity is maintained to provide flexibility in any future versions of MELTER that might incorporate additional detail in the treatment of inert cargo.
} 
and lleft(2) are the numbers of spherical layers that remain for damage states 1 and 2. Damage state 0 corresponds to undamaged cargo, and damage state 3 corresponds to cargo for which the critical layer (identified through ncs 0 ) is the external layer presented to the rest of the enclosure analysis.

\subsection{Output}

As used in the PRA, MELTER's principal product is the file cargo.MEL which it creates. For each scenario analyzed the following values are written to cargo.MEL: 1) the minimum critical fire duration, 2) the corresponding quantity of fuel for that scenario which is proportional to fire diameter times duration. and 3-7) the indices of the parameters read from scenes. BRC that define the scenario. In the event that no failure can be predicted for any fire duration arbitrarily large $\left(10^{7}\right)$ values are written to the fire duration and fuel quantity fields. The PRA analyst must coordinate the construction of scenes. BRC and subsequent use of cargo.MEL. ${ }^{11}$

Table 4 includes the lines from MELTER which produce cargo. MEL. quest 1 is a logical variable which is .true. if the fire duration has been specified. In that case three values are written to cargo.MEL. The first value is a ' 1.0 ' or ' 2.0 ' indicating, respectively, failure or survival. If a failure has resulted the time of that failure and the fire duration are reported. If the cargo survives the time at which the analysis was halted and the temperature of the critical layer at that point in time are reported.

MELTER also produces files that contain information useful to assess the consistency of its results. cargo.STA contains the number of failures and average value of the critical fire duration for each index of each parameter specified in scenes. BRC. For example, if the most distant fires specified in scenes. BRC never lead to failures, the range of that parameter should be decreased to improve resolution. Cases have been encountered for which the critical fire duration is difficult to determine because failure occurs much later than the extinction of the fire. If a really obstinate scenario is encountered MELTER can move on to the next case without successfully resolving the critical fire duration. In those cases MELTER writes to cargo.XXX the sequence of iflag values that were returned by ode, the integrator, during its attempt to solve that problem. There were no such occurrences for 29 of the 33 cargos, two cargos produced a single case in which a solution was not found, and two cargos produced about $5 \%$ exceptions, which were invariably associated with distant, small fires. These failures result when difficulty is encountered in integrating the equations. The individual cases were resolved by running these same cargos with a version of MELTER that used a simple finite-difference approach to integrating the equations (which was more robust but slower). MELTER's

\footnotetext{
${ }^{11}$ The PRA analysis knows the fire diameter corresponding to each value in cargo. MEL. The probability of sufficient fuel existing to reach the critical fire duration is bottom line used in determining the probability of fire-induced failures.
} 
output was then repaired with numbers derived with the finite difference version of the code. $^{12}$

If noutput $>0$, as set in cargo.TAR, and the fire duration has been specified MELTER generates an additional file MELTER. OUT. This file includes intermediate results calculated such as view factors and the distribution associated with $A_{1 . .9}$. The MELTER. OUT file associated with Case I of the results section is included as Appendix B.

\footnotetext{
${ }^{12}$ Results for cases solved by both integrators were verified to be in close agreement.
} 
Section that writes cargo.MEL:

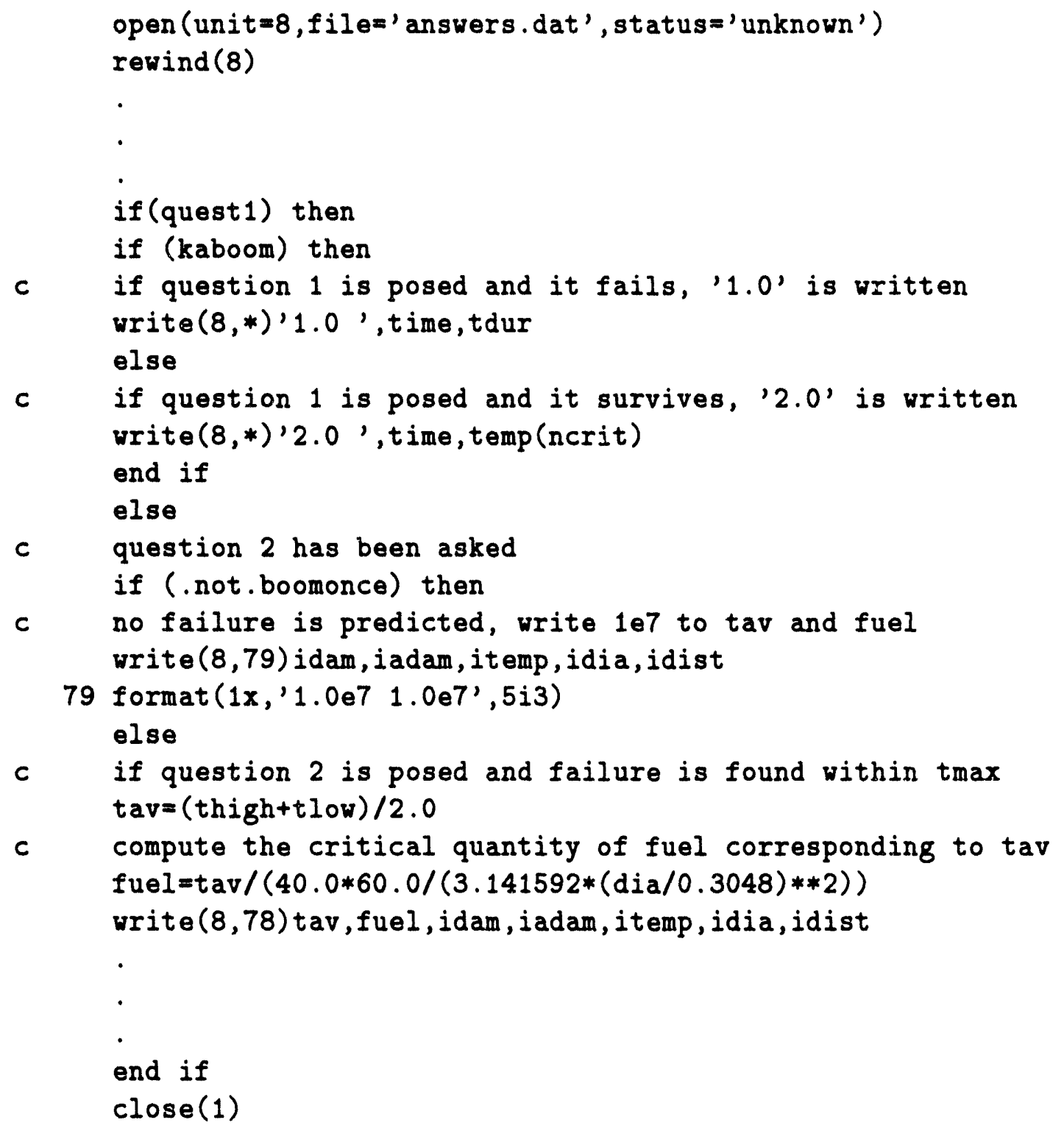

Table 4: Code section that writes results. 


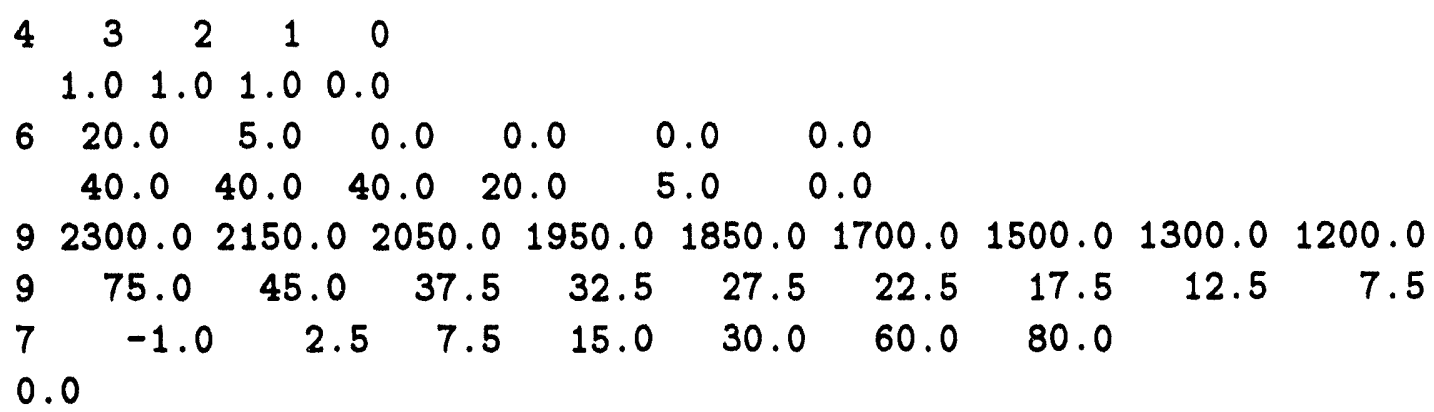

Table 5: DPTRA.BRC, the scenes. BRC file used to defined scenarios considered in results reported in Table 6.

\section{MELTER's Performance in PRA}

At this writing MELTER has been used on 33 different cargos in combination with a set 13,608 scenarios which span the parameter space of interest. These results have been inspected for consistency and reasonableness. The results produced are consistent within the resolution reasonably expected of the present analysis. As described in Section 4.3.1, scenes. BRC defines discrete values for cargo damage, SST damage, fire temperature, fire diameter, and fire displacement. Table 5 includes, DPTRA.BRC, the scenario input that was used to generate results discussed in this section. In DPTRA.BRC the parameters are nominally ordered from most to least severe. Each cargo analyzed in concert with DPTRA.BRC resulted in a 13,608-line cargo.MEL file. These files have been scanned with a short computer program to observe whether adjacent cases exhibit the expected trends. A pair of scenarios sharing all the same parameters except one, that parameter being one increment displaced, are said to be adjacent. Over 50,000 such adjacent pairs result for the set of scenarios in question. Each pair is examined and exceptions from what is expected are noted. Depending on the cargo being analyzed, from 0 to $5 \%$ of these comparisons yield results departing from the nominal expectation. The sources and expected consequences of these departures are discussed in this section. Table 6 summarizes the results of this scanning activity for the cases that have been analyzed as of this writing. The first three columns of Table 6 correspond to comparisons associated with the distance, diameter, and temperature of the fire; the next two columns deal with the damage condition of the SST and of the cargo; and the last column is the average result for all comparisons. For example, the first column reports the percentage of cases in which an increase in fire distance did not yield the expected increase in critical fire duration. Several types of apparent anomalies are produced including: 
1. Some adjacent scenarios are virtually equivalent and acceptable numerical uncertainty produces some inconsistency.

2. The cargo in a more damaged SST may require longer fire duration to induce failure than that in a less damaged SST-all other things being equal.

3. Fires of greater diameter produce no failure or less rapid failure when those of lesser diameter have been producing failures.

4. Increasing the damage of the cargo does not always result in a shortening of the failure time.

It should be noted that the results reported in Table 6 are dependent upon the resolution with which the parameter space is discretized. If each parameter were changed only in very significant increments, Table 6 would be a table of 0 's. The following sections discuss the apparent inconsistencies that were created using the parameter space defined by DPTRA. BRC given in Table 5.

\subsection{Numerical Uncertainty}

MELTER hunts for the minimum fire duration that will result in a thermal failure condition. A bisection method is employed to narrow the interval of uncertainty. The accepted interval of uncertainty is the lesser of $1 \%$ of the fire duration or 50 seconds. When two scenarios are very close to equivalent but not identical the resulting minimum critical time estimates will be very close to each other and not necessarily ordered as expected. This numerical uncertainty creates many of the discrepancies reported in Table 6 including all of those of the first column.

\subsection{Distance}

Referring to Table 6, very few inconsistencies are associated with fire separation distance. About $3 / 4$ of those that do occur arise when comparing a SST in an engulfing fire to one with a large fire just against the side of the SST. For cases in which the SST is damaged, the damaged area of the SST is uniformly covered by either of these fires. As a consequence they are virtually equivalent threats to the cargo and, coupled with numerical uncertainty, give rise to an occasional inconsistency. No significant effect on the overall probability of failure can reasonably be associated with these inconsistencies.

\subsection{Fire Diameter}

A significant source of this type of discrepancy also results from the fact that for some scenarios additional fire diameter really is virtually inconsequential. For fast- 


\begin{tabular}{||l|r|r|r|r|r||r||}
\hline \hline Cargo & dist & dia & temp & $\begin{array}{r}\text { sst } \\
\text { damage }\end{array}$ & $\begin{array}{r}\text { cargo } \\
\text { damage }\end{array}$ & Average \\
\hline \hline comp1 & 0.0 & 4.3 & 0.0 & 4.4 & 0.0 & 1.8 \\
comp2 & 0.0 & 4.7 & 0.0 & 3.0 & 18.5 & 4.9 \\
comp3 & 0.0 & 3.1 & 0.0 & 2.7 & 0.0 & 1.2 \\
comp4 & 0.0 & 5.0 & 0.0 & 10.0 & 0.0 & 3.0 \\
comp5 & 0.5 & 9.4 & 0.7 & 5.5 & 4.8 & 4.1 \\
comp6 & 0.0 & 5.4 & 0.1 & 5.7 & 0.9 & 2.4 \\
comp7 & 0.0 & 4.8 & 0.0 & 10.7 & 2.8 & 3.6 \\
comp8 & 0.0 & 7.7 & 0.0 & 3.2 & 2.7 & 2.7 \\
comp9 & 0.0 & 5.6 & 0.1 & 2.2 & 0.0 & 1.6 \\
comp10 & 0.1 & 4.8 & 0.2 & 7.1 & 0.0 & 2.5 \\
comp11 & 0.1 & 5.0 & 0.4 & 10.6 & 0.2 & 3.3 \\
comp12 & 0.1 & 5.2 & 0.3 & 9.7 & 0.0 & 3.1 \\
comp13 & 0.0 & 5.2 & 0.1 & 9.8 & 0.6 & 3.2 \\
comp14 & 0.0 & 5.9 & 0.0 & 4.4 & 1.5 & 2.4 \\
comp16 & 0.2 & 5.8 & 0.2 & 8.7 & 0.0 & 3.0 \\
comp17 & 0.0 & 5.1 & 0.0 & 5.7 & 1.2 & 2.4 \\
north1 & 0.0 & 4.8 & 0.1 & 14.1 & 0.5 & 3.9 \\
north2 & 0.1 & 4.1 & 0.4 & 14.3 & 0.4 & 3.9 \\
north3 & 0.3 & 4.6 & 0.0 & 14.1 & 0.4 & 3.9 \\
north4 & 0.0 & 4.7 & 0.0 & 8.4 & 2.7 & 3.1 \\
north5 & 0.1 & 4.9 & 0.0 & 8.1 & 1.0 & 2.8 \\
north7 & 0.0 & 4.5 & 0.0 & 12.6 & 0.5 & 3.5 \\
north8 & 0.1 & 6.2 & 0.0 & 8.3 & 5.3 & 3.9 \\
north9 & 0.0 & 4.5 & 0.0 & 12.7 & 0.5 & 3.6 \\
west1 & 0.2 & 3.7 & 0.3 & 3.0 & 7.3 & 2.8 \\
west2 & 1.2 & 8.1 & 0.1 & 8.6 & 3.4 & 4.3 \\
west3 & 1.1 & 4.5 & 0.3 & 19.9 & 2.1 & 5.5 \\
west4 & 0.3 & 5.3 & 0.2 & 6.1 & 15.4 & 5.2 \\
west7 & 0.4 & 3.9 & 0.2 & 10.5 & 0.3 & 3.1 \\
west8 & 0.1 & 4.4 & 0.3 & 13.4 & 0.6 & 3.8 \\
west10 & 0.1 & 5.5 & 0.2 & 6.6 & 0.9 & 2.7 \\
west11 & 2.8 & 6.7 & 0.9 & 18.3 & 1.1 & 6.0 \\
west12 & 0.2 & 4.6 & 0.0 & 17.5 & 0.2 & 4.5 \\
\hline average & 0.2 & 5.2 & 0.2 & 9.1 & 2.3 & 3.4 \\
\hline \hline
\end{tabular}

Table 6: Summary of $\%$ of adjacent scenarios not exhibiting expected trend. 
failing cases involving a damaged SST, once the fire is big enough to cover $A_{8}$ and/or $A_{9}$, additional fire size has little influence. Under these conditions, additional $A_{7}$ is of no important consequence. These diameter inconsistencies result from numerical uncertainty. That many of these arise from numerical uncertainty is supported by the fact that comparatively many inconsistencies associated with diameter occur for the largest couple of diameters considered at the engulfing and abutting separations.

Circumstances can arise which MELTER does not resolve correctly. Refer to Figure 15a, and regard the cargo as being isolated in the middle of the SST with an unobstructed view to the heated walls of the SST. $A_{1}$ sees both $A_{7}$ and $A_{8}$. Consider first a case for which a specified $A_{8}$ is just neatly covered by a fire adjacent to the SST. $A_{8}$ will be aligned with the fire and there will also be a finite area, $A_{7}$. Referring still to Figure $15 \mathrm{a}$, consider an increase in the fire size while maintaining the same $A_{8}$. The effect is an increase in $A_{7}$. Since $A_{7}$ 's thermal response lags that of $A_{8}, A_{1}$ is relieved because the viewfactor-weighted average temperature of the surface seen by $A_{1}$ is lower than in the first case.

\subsection{SST Damage}

\subsubsection{Stored Energy Discharge}

Nominally, we expect increasing damage to a SST to make it more vulnerable to failure. However, it is physically reasonable that a more damaged SST will have a better chance of survival than a less damaged SST for certain fire and cargo conditions. Suppose a fire of fixed size and duration threatens a damaged SST. If the fire is short and intense it will store a significant amount of energy in the SST before the cargo has an opportunity to respond. After the fire is out a lot of energy has been trapped in the SST's thermal capacitance. For this scenario it is entirely possible that the more damaged SST not lead to failure because of the discharge of that stored energy to the environment before a critical thermal response is induced in the cargo. The physics that are included in MELTER can predict this behavior. This effect accounts for some of the SST damage anomalies.

\subsection{2 $A_{8}$ Nonuniformity}

Another source of aberration results from limitations of the enclosure heat transfer analysis. Refer to Figures $15 \mathrm{a}$ and $15 \mathrm{~b}$ which represent two cases having identical $A_{1}$, the "sunny side" area of the cargo by virtue of "seeing" the same total amount of heated armor $A_{7}+A_{8} . A_{7}$ is the area of nominal wall construction directly heated by the fire. $A_{8}$ is the area of wall with the exterior skin and rigid foam removed that is directly heated by the fire. The geometries shown share the feature that the cargo is close to the wall (proximity=1). It seems clear enough that Figure 15b depicts 


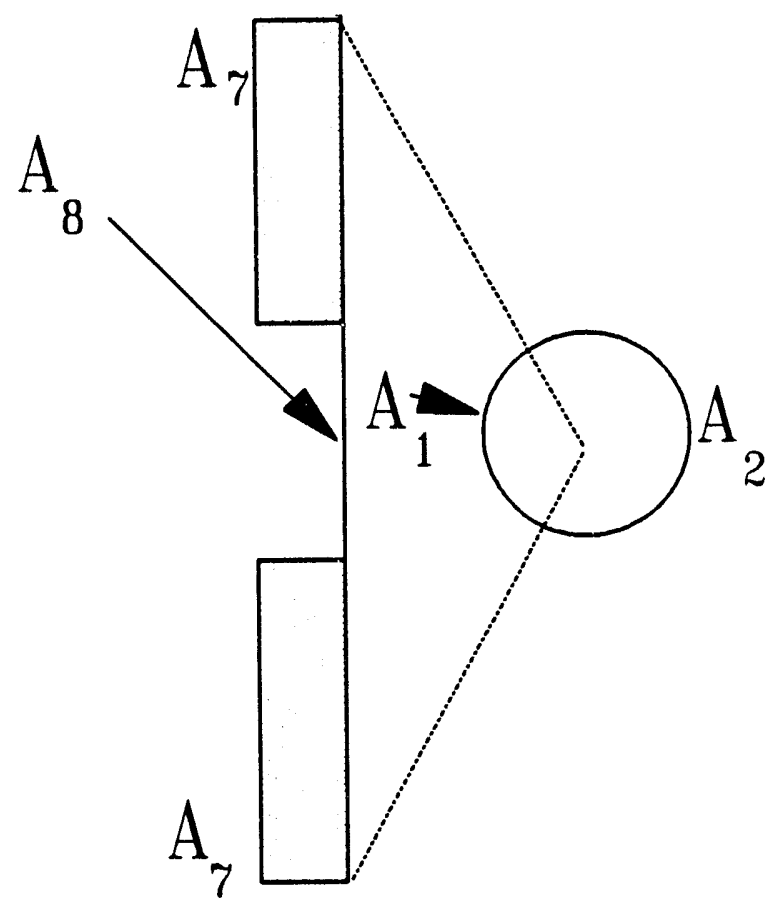

Fig 15a

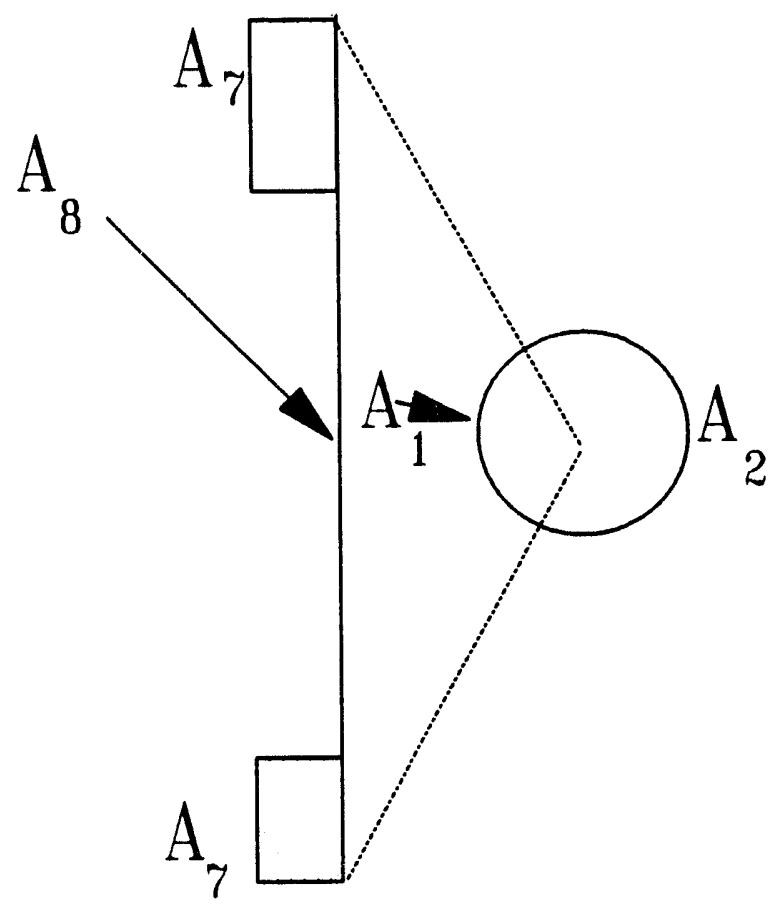

Fig 15b

Figure 15: Example of two geometries that illustrate a model limitation. 
the more threatening condition and for most fire scenarios MELTER will predict just that. However, for scenarios with a rather severe fire condition, MELTER will generally predict faster ignition for Figure 15a's geometry. The reason is that in Figure $15 \mathrm{~b} A_{8}$ views both the exterior of the cargo and other thermal capacitance within the SST. In Figure $15 \mathrm{a}, A_{8}$ tends towards seeing only $A_{1}$. As a result, $T_{A_{8}}(t)$, the temperature history of $A_{8}$ associated with Figure $15 \mathrm{~b}$ will exhibit a more sluggish response than that associated with Figure 15a. The situation violates the model's idealization that $A_{8}$ be spatially isothermal. To remove this discrepancy (perhaps a future version of) MELTER would have to incorporate additional area elements in the radiation enclosure so that a distribution of temperature for $A_{8}$ can be resolved. Most of the nonmonotonic results associated with SST damage are due to this effect. Considering all cargos together, nearly $70 \%$ of these inconsistencies occur when comparison is made between the SST with $A_{8}=20 \mathrm{ft}^{2}$ and that with $A_{8}=5 \mathrm{ft}^{2}$. Given the uncertainty associated with specifying a distribution of $A_{8}$, MELTER's results are acceptably consistent.

\subsection{Cargo Damage}

This type of inconsistency is primarily due to numerical uncertainty coupled with conditions for which subsequent damage states are of little consequence. The crude discretization that is used in representing the cargos gives rise to this situation. Often the thermal models representing successive damage states differ by a single spherical annulus. A given single annulus of material within the model is spatially uniform in temperature. As a result the delay we expect as thermal energy diffuses through the system is consistently underestimated. In some cases an increment of damage to the cargo will result in eliminating a conduction connector of the type indicated by Eq. (19) and a radiation connector of the type indicated by Eq. (20). The newly exposed exterior surface of the cargo has less surface area than that associated with the next less damaged state. The thermal impedance from the outside of the cargo can actually increase. $^{13}$

\footnotetext{
${ }^{13}$ This is similar to the counter-intuitive result that the addition of insulation to a simple pipeinsulation-convection system can increase the steady heat transfer. See Ref. [8].
} 


\section{Concluding Remarks}

The MELTER model of the thermal response of cargos transported in a SST subject to fire environments and its implementation in a computer code have been developed. MELTER estimates the minimum fire duration creating a critical condition for a cargo in a SST in a fire. A satisfactory tool to be employed in PRA has been developed. Salient features of MELTER include:

- The existing model accommodates a wide variety of fire scenarios. MELTER incorporates the sampling of the user-specified parameter space.

- Unique cargo.TAR files are loaded into the analysis just as unique cargos are loaded into SSTs. Furthermore, the cargo can be rendered more vulnerable by provisions for damage states.

- Means to reflect damage to the SST have been incorporated.

- Qualitatively satisfying results have been obtained. Bigger, hotter, and closer fires lead to shorter failure times as expected (see Section 5.3).

- MELTER is robust. On the order of a million scenarios/cargo combinations have been run.

- Affordable execution times have been achieved.

- A balanced level of modeling detail is maintained.

- Conservative assumptions have been incorporated when necessary. 


\section{$7 \quad$ References}

1. Ellingsen, Kjetil, "Thermal Radiation from Hydrocarbon Pool Fires," MS Thesis, South Dakota School of Mines and Technology, May, 1992.

2. Modak, Ashok T., "Thermal Radiation from Pool Fires," Combustion and Flame, 29, pp. 177-192, 1977.

3. Siegel, R. and J. R. Howell, Thermal Radiation Heat Transfer, 2nd Edition, McGraw Hill, 1981.

4. Laub, B., Chiba, Z., Fretter, E. F., Beck, R.A.S., Trupact Insulation Evaluation, Acurex Corporation, July, 1983.

5. Rein, Anders, "A Study of the Transient Thermal Response of a Slab of Rigid Foam Subjected to a Fire Boundary Condition," MS Thesis, South Dakota School of Mines and Technology, December, 1991.

6. Nakos, J. T., and B. G. Strait, Data Representation and Interpretation from SST Test \# 2, Sandia National Laboratories internal Memorandum, August, 1990.

7. Andersen, Runa H., "Thermal Analysis of Transient Radiation and Conduction in a Thick-walled Enclosure," MS Thesis, South Dakota School of Mines and Technology, December, 1992.

8. Holman, J. P., Heat Transfer, 7th Edition, McGraw Hill, 1990.

9. Fehlberg, E., "Low-Order Classical Runge-Kutta Formulas with Stepsize Control and Their Application to Some Heat Transfer Problems," NASA TR R-315, 1969.

10. Shampine, L. F., and H. A. Watts, "A smoother Interpolant for DE/STEP, INTRP and DEABM," SAND83-1226.

11. Stoecker, W. F., Design of Thermal Systems, 3rd edition, McGraw-Hill, 1989. 


\section{A Parameter Optimization for the Charring Wall Model}

This appendix discusses the optimum selection of parameters for the wall model used in MELTER. Figure 16 summarizes data from the 2nd SST Panel Burn Test (Ref. [6]). Shown are the fire temperature at one location and the average armor temperature at each of three different elevations. The block in the upper right hand corner of the plot also shows the thermocouple locations. Clearly, there is vertical stratification in the armor heating. We know that the fire temperatures outside the wall also exhibit this trend and that is the most likely explanation. However, some of this height dependency may also be the result of differences in the condition of the wall resulting from its nonuniform destruction. In any case, we are presently stuck with fitting a one-dimensional model to these results. What is the best temperature history to ask our model to try and duplicate?

Figure 17 shows the average temperatures at the three elevations again along with two methods of averaging those temperatures. The simplest method is the arithmetic average of all nine locations. The other temperature is that which exhibits the same average emissive power as all nine thermocouple locations. That is:

$$
\bar{T}=\left(\frac{1}{9} \sum_{i=1}^{9} T_{i}^{4}\right)^{1 / 4}
$$

This yields a higher temperature than simple arithmetic averaging and it is the temperature we should strive to duplicate. This is because it is emissive power rather than temperature that is proportional to the amount of heat we expect to be transferred into the enclosure.

A univariate search method (Ref. [11]) was developed to find the optimum set of: $T_{f}, a_{w}, \Delta E_{g a s s i f y}$, and $T_{\text {react }}$ for the wall model described in Section 2.3. The goal of the search is to find values for the parameters that will minimize:

$$
H=\sum_{i}\left(\phi_{i}-\theta_{i}\right)^{2}
$$

where $\phi_{i}$ and $\theta_{i}$ represent the data ${ }^{14}$ and the prediction using the simple wall model.

Searches like these are often not well-behaved and that was the case for this one. The search for optimum $T_{f}$ and $T_{\text {react }}$ were abandoned. $T_{f}$ was fixed at $1280 \mathrm{~K}$, which is the average of the fire temperature shown in Figure 16. $T_{\text {react }}$ was fixed at $585 \mathrm{~K}$. The search meanders around in $a_{w}-\Delta E_{\text {gassify }}$ space and fails to expose a sharply defined optimum.

\footnotetext{
${ }^{14}$ The average temperature calculated using Eq. (A1).
} 


\section{SST Panel Test 2 Temperatures Avg. @ each elevation \& Cent. Twr. @ 8'}

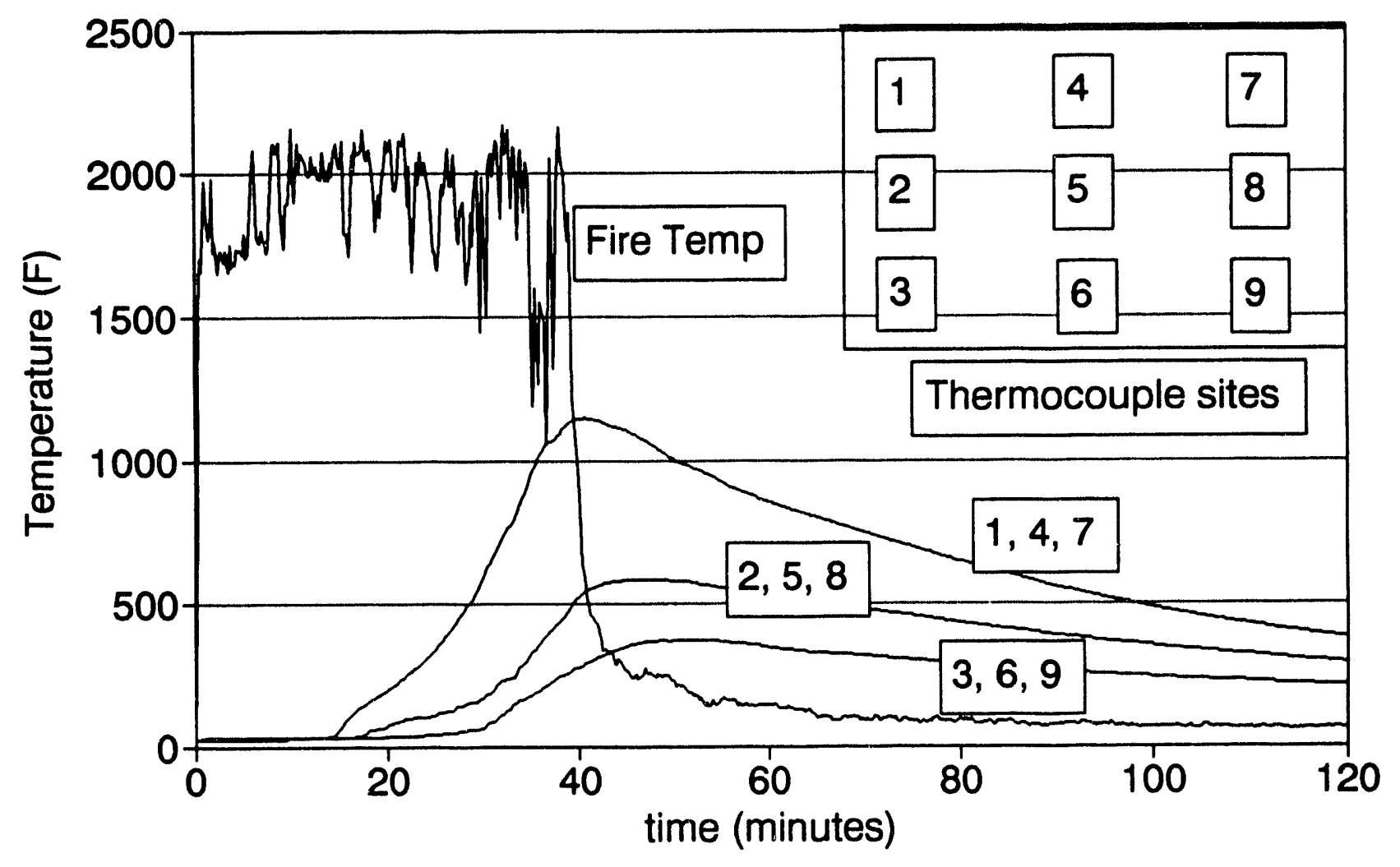

Figure 16: Temperature histories from 2nd SST wall panel burn test. 


\section{SST Panel Test 2 Temperatures Avg. T\& $T$ with avg. $E$}

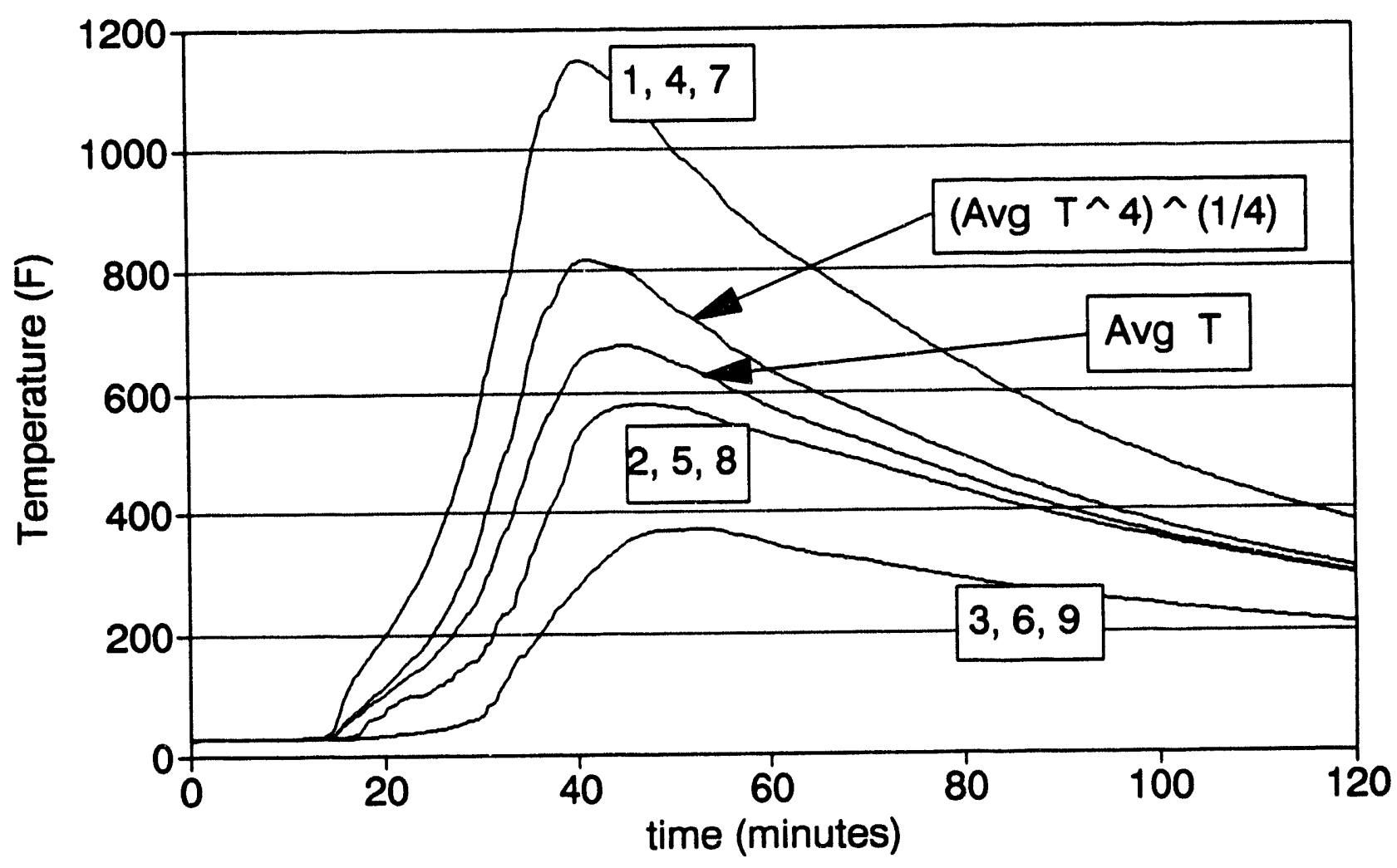

Figure 17: Temperature averages from 2nd SST wall panel burn test. 


\begin{tabular}{||l|r|r||}
\hline \hline $\begin{array}{l}\text { Fitting period } \\
(s)\end{array}$ & $\begin{array}{r}a_{w} \\
\left(\mathrm{~mm}^{-1}\right)\end{array}$ & $\begin{array}{r}\Delta E_{\text {gassify }} \\
\left(\mathrm{J} / \mathrm{m}^{3}\right)\end{array}$ \\
\hline \hline $0-2000$ & 0.055 & 70,400 \\
\hline $0-5000$ & 0.051 & 68,500 \\
\hline \hline
\end{tabular}

Table 7: Results of optimum wall parameter search.

Figure 18 shows the model and data for one of the best fits found when requiring the model to match the first 2000 seconds of the data. Figure 19 shows those same results when the matching period is extended to 5000 seconds, well into the cooling of the wall after the fire dies. ${ }^{15}$ The fits of Figs. 18 and 19 are visually pleasing. Finally, Figure 20 shows the 5000 second period using the optimum parameters from the 2000 second fitting period. The increased discrepancy following the 2000 second interval is fairly visible. Table 7 summarizes the results of these searches.

It is encouraging that $a_{w}$ and $\Delta E_{g a s s i f y}$ remain in the same neighborhood when optimizing periods of either 2000 or 5000 seconds are chosen. That the optimum is evasive is easily understood in terms of the physics of the problem. The time delay before the armor begins to heat is increased by increasing either of $a_{w}$ or $\Delta E_{\text {gassify }}$. Consequently, this feature of the graph can be matched well with nearly any value we like for one of the parameters, provided we adjust the other. On the other hand, following the charring of the wall the slope of the armor temperature should be almost exclusively dependent upon $a_{w}$ which should help define $a_{w}$ fairly well without regard for the other parameter. It is easy to see that $T_{\text {react }}$ would not be well-defined for this problem because the emissive power of the wall's skin temperature is much greater than that at the reaction front. As a consequence, it does not matter much what we call $T_{\text {react }}$ and including it only muddied the waters. If we had comparable data for conditions that led to $T_{\text {skin }}$ approaching $T_{\text {react }}, T_{\text {react }}$ would become well-defined.

Referring to Table 7, the parameters that result from the longer averaging period are more conservative. That is, lower $\Delta E_{g a s s i f y}$ and lower $a_{w}$ both favor comparatively rapid heat transfer through the wall. In keeping with the policy of conservatism, those parameters have been implemented in MELTER.

\footnotetext{
${ }^{15}$ The cooling of the wall through its insulated backside is also estimated.
} 


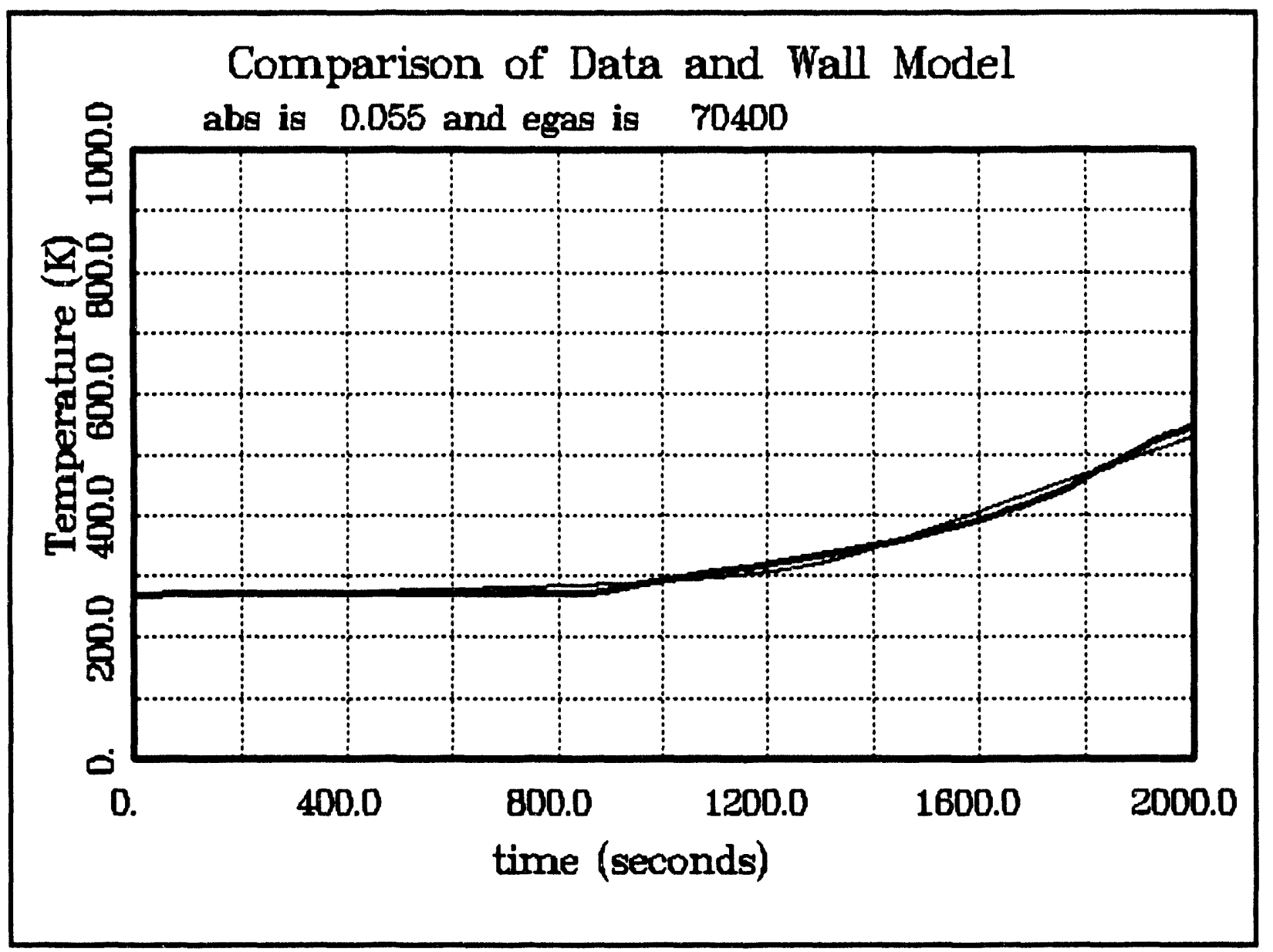

Note: the heavier curve is the data and the lighter curve is the model prediction.

Figure 18: Comparison of data and wall model using parameters optimized to the first 2000 seconds of data. 


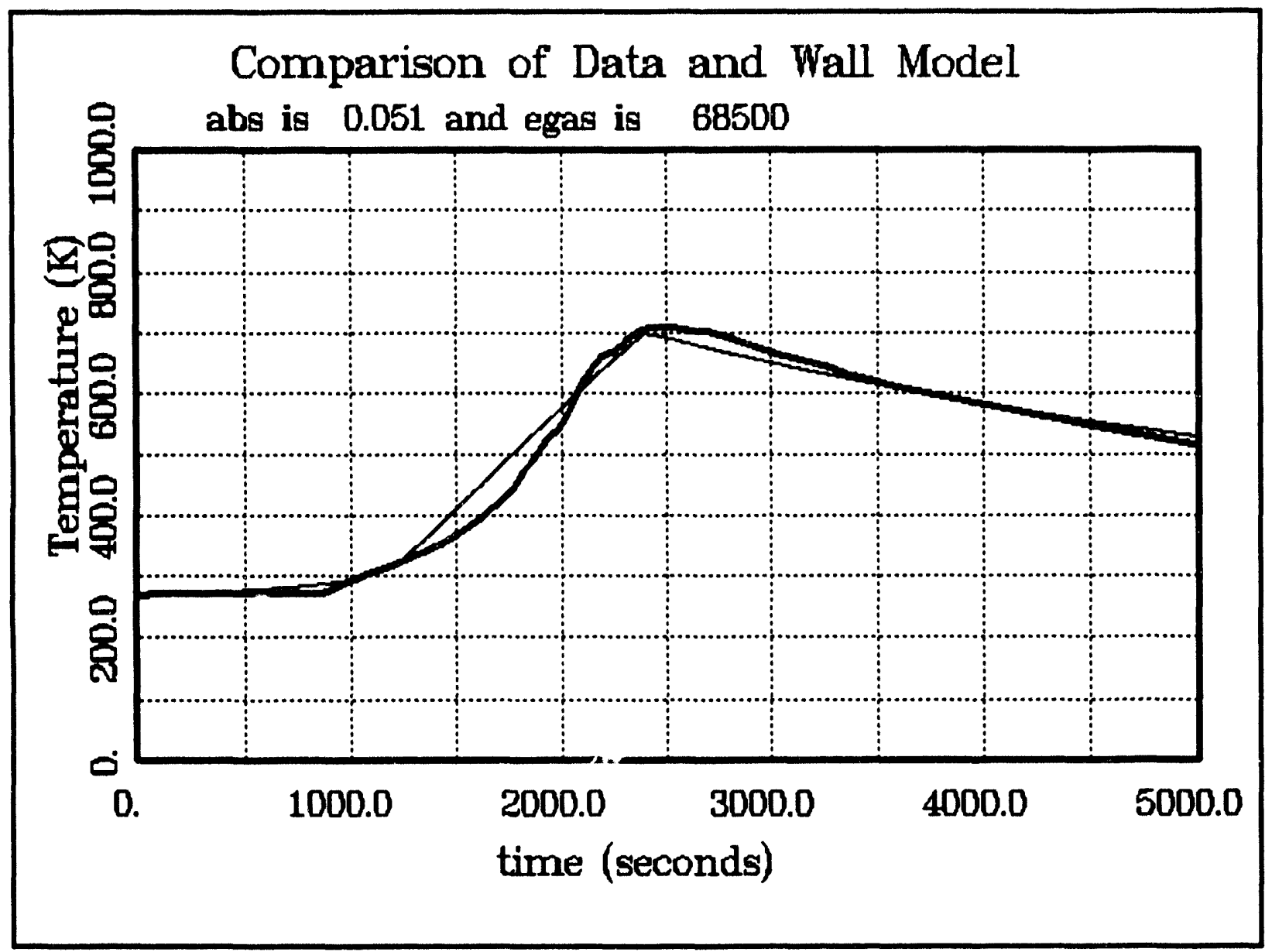

Note: the heavier curve is the data and the lighter curve is the model prediction.

Figure 19: Comparison of data and wall model using parameters optimized to the first 5000 seconds of data. 


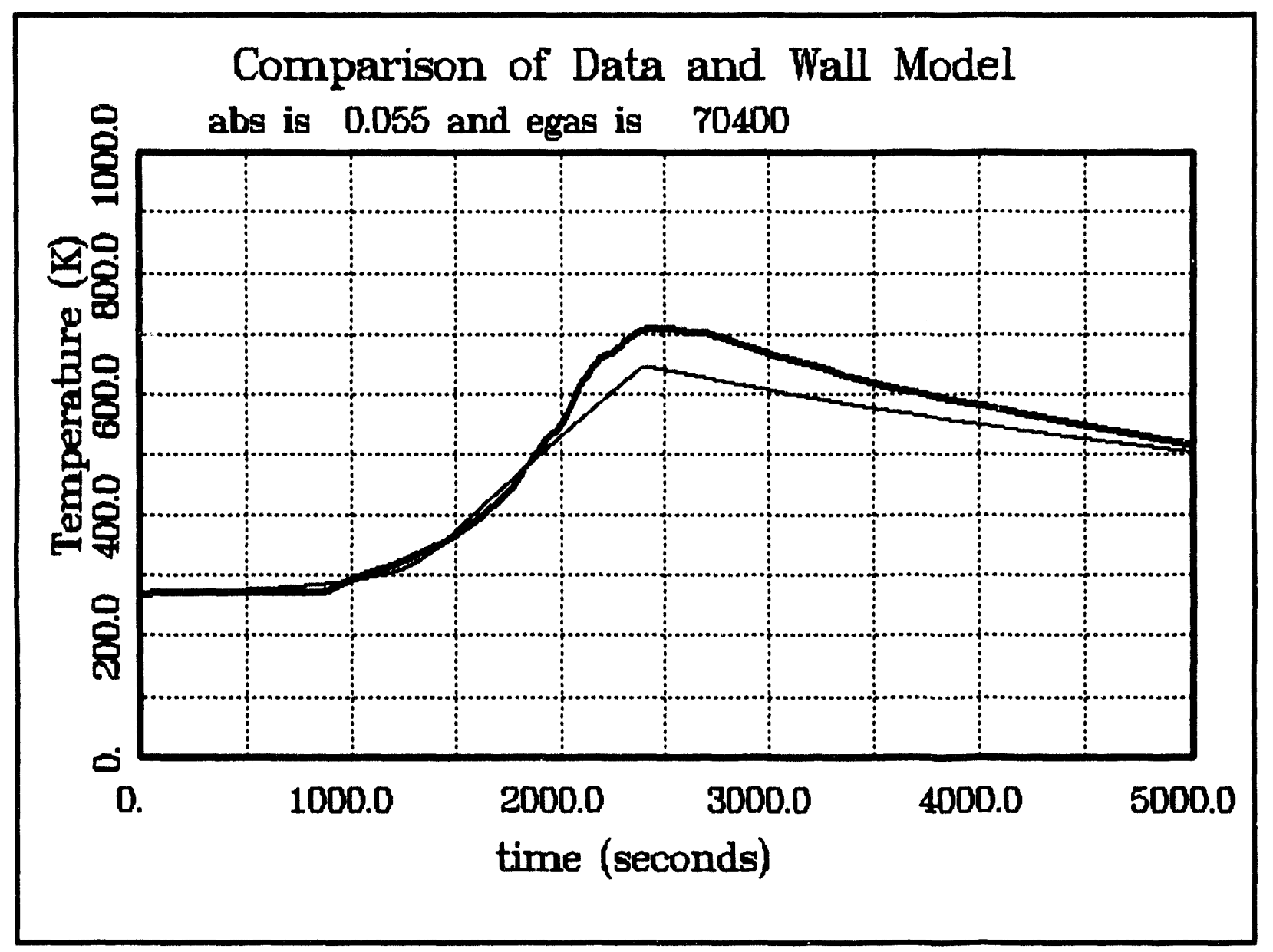

Note: the heavier curve is the data and the lighter curve is the model prediction.

Figure 20: Comparison of data and wall model through 5000 seconds with parameters optimized to the first 2000 seconds of data. 


\section{B MELTER . OUT File for Case I}

The view factors used in the enclosure analysis

$\begin{array}{lllllll}.0000 & .0000 & .4000 & .0000 & .1610 & .0000 & .4390 \\ .0000 & .0000 & .2000 & .0822 & .0000 & .7178 & .0000 \\ .0123 & .0020 & .9226 & .0014 & .0132 & .0125 & .0359 \\ .0000 & .0730 & .1270 & .0000 & .0000 & .1808 & .6192 \\ .0548 & .0000 & .1452 & .0000 & .0000 & .2210 & .5790 \\ .0000 & .0730 & .1270 & .0207 & .2031 & .0000 & .5762 \\ .0548 & .0000 & .1452 & .0283 & .2124 & .2299 & .3294 \\ \text { surface emis8ivities are: } & & & & \\ .7000 & .7000 & .7000 & .7000 & .7000 & .7000 & .7000 \\ \text { l7ter Run at } 13: 40 \text { on 6- 7-93 } & & & & \end{array}$

The total number of stace variables is

The cargos number of concentric shells

The cargos number of temperatures

The sunny side of the cargo has area

The dark side of the cargo has area

Inerts have area

Floor area not directly exposed

Floor area directly exposed

Wall interior not directly exposed Normal wall directly exposed Armor with foam removed Hole area

Distance from the fire is

The fire diameter $(m)$ is

The view of $a(7 . .9)$ to fire is

The specified fire duration is
21

6

11

6.172

2.008

200.000

2.259

18.141

19.738

49.462

.000

.000

.000

9.144

1.000

1800.000

Output is ordered:

time, $T(1) \ldots T$ (ncargo), $T(A 3) \ldots T(A 8), T$ skin(A7), $\quad x$ char(A7), Tskin(A5), xch

$\operatorname{ar}(A 5)$

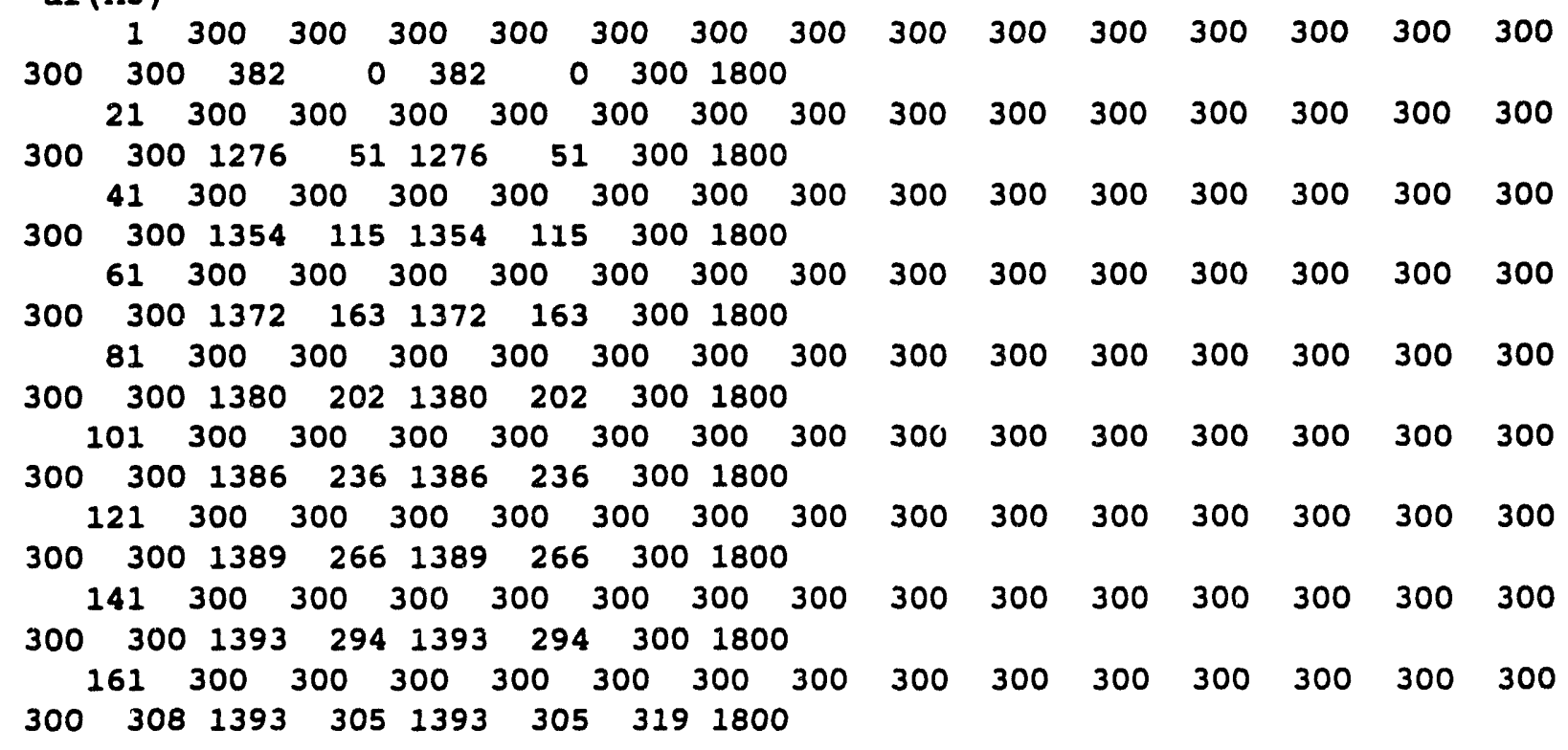


194300300300300300300300 $300 \quad 327 \quad 1392 \quad 305 \quad 1392 \quad 305 \quad 361 \quad 1800$

$\begin{array}{lllllllllllllll}226 & 300 & 300 & 300 & 300 & 300 & 300 & 300 & 300 & 300 & 304 & 301 & 300 & 300 & 302\end{array}$

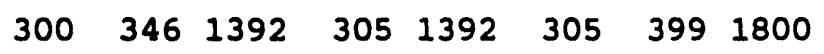

$\begin{array}{lllllllllllllll}290 & 300 & 300 & 300 & 300 & 300 & 300 & 300 & 300 & 300 & 313 & 304 & 300 & 301 & 308\end{array}$

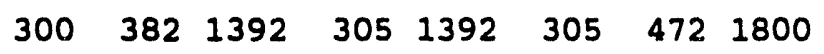

$\begin{array}{lllllllllllllll}418 & 300 & 300 & 300 & 300 & 300 & 300 & 300 & 306 & 301 & 349 & 317 & 301 & 305 & 327\end{array}$

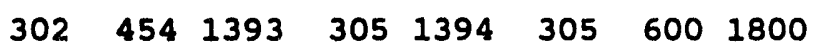

$\begin{array}{lllllllllllllll}674 & 300 & 300 & 300 & 300 & 300 & 301 & 300 & 352 & 315 & 457 & 370 & 310 & 329 & 391\end{array}$

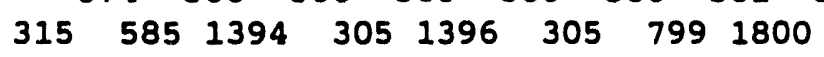

$\begin{array}{lllllllllllllll}974 & 300 & 303 & 300 & 306 & 301 & 313 & 303 & 476 & 366 & 567 & 451 & 337 & 399 & 499\end{array}$

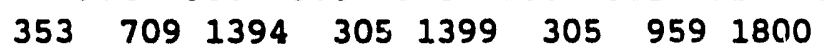

$\begin{array}{lllllllllllllll}1157 & 301 & 308 & 302 & 316 & 304 & 329 & 307 & 550 & 415 & 621 & 498 & 365 & 465 & 573\end{array}$

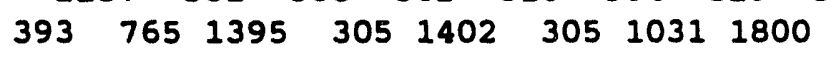

$\begin{array}{lllllllllllllll}1295 & 303 & 315 & 304 & 326 & 307 & 344 & 312 & 591 & 456 & 655 & 532 & 392 & 524 & 627\end{array}$ $\begin{array}{llllllll}431 & 799 & 1396 \quad 305 & 1402 & 305 & 1075 & 1800\end{array}$

$\begin{array}{lllllllllllllll}1429 & 307 & 323 & 307 & 338 & 312 & 361 & 320 & 624 & 495 & 684 & 565 & 423 & 583 & 677\end{array}$ $\begin{array}{lllllll}473 \quad 826 & 1396 \quad 305 \quad 1403 \quad 305 \quad 1111 \quad 1800\end{array}$

$\begin{array}{lllllllllllllll}1558 & 311 & 333 & 312 & 351 & 319 & 378 & 330 & 651 & 532 & 709 & 599 & 458 & 637 & 721\end{array}$

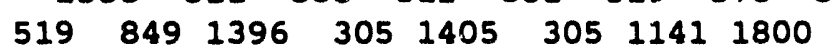

$\begin{array}{lllllllllllllll}1678 & 316 & 343 & 318 & 364 & 327 & 395 & 341 & 674 & 566 & 732 & 630 & 493 & 683 & 756\end{array}$ $\begin{array}{lllllll}564 \quad 866 & 1397 \quad 305 & 1406 & 305 \quad 1165 & 1800\end{array}$

$\begin{array}{lllllllllllllll}1785 & 321 & 353 & 325 & 377 & 335 & 412 & 354 & 693 & 596 & 751 & 660 & 527 & 720 & 784\end{array}$ $\begin{array}{llllllll}605 & 881 & 1397 & 305 & 1407 & 305 & 1183 \quad 1800\end{array}$

$\begin{array}{lllllllllllllll}1800 & 322 & 354 & 326 & 378 & 337 & 414 & 356 & 695 & 600 & 753 & 664 & 532 & 724 & 788\end{array}$ $\begin{array}{llllllll}611 & 883 & 1397 & 305 & 1407 & 305 & 1185 & 1800\end{array}$

$\begin{array}{lllllllllllllll}1891 & 327 & 364 & 333 & 390 & 346 & 427 & 368 & 700 & 622 & 747 & 678 & 560 & 745 & 803\end{array}$ $\begin{array}{llllllll}642 & 852 & 583 & 305 & 648 & 305 & 1104 & 1800\end{array}$

$\begin{array}{lllllllllllllll}2075 & 340 & 382 & 349 & 407 & 366 & 441 & 391 & 688 & 645 & 731 & 696 & 605 & 755 & 805\end{array}$ $\begin{array}{llllllll}685 & 804 & 476 & 305 & 554 & 305 & 980 & 1800\end{array}$

$\begin{array}{lllllllllllllll}2375 & 362 & 405 & 379 & 428 & 398 & 459 & 426 & 678 & 665 & 722 & 713 & 655 & 745 & 784\end{array}$

$\begin{array}{llllllll}718 & 761 & 439 & 305 & 492 & 305 & 864 & 1800\end{array}$

$\begin{array}{lllllllllllllll}2675 & 386 & 426 & 408 & 447 & 428 & 476 & 457 & 676 & 674 & 717 & 719 & 684 & 735 & 763\end{array}$

$\begin{array}{llllllll}727 & 738 & 424 & 305 & 457 & 305 & 798 & 1800\end{array}$

$\begin{array}{lllllllllllllll}2975 & 408 & 445 & 435 & 466 & 454 & 493 & 482 & 675 & 677 & 716 & 719 & 700 & 727 & 745\end{array}$

$\begin{array}{llllllll}727 & 724 & 417 & 305 & 435 & 305 & 755 & 1800\end{array}$

$\begin{array}{lllllllllllllll}3024 & 412 & 449 & 439 & 469 & 458 & 496 & 486 & 675 & 678 & 716 & 719 & 702 & 726 & 743\end{array}$

$\begin{array}{llllllll}727 & 722 & 416 & 305 & 432 & 305 & 750 & 1800\end{array}$

$\begin{array}{lllllllllllllll}3067 & 415 & 451 & 442 & 471 & 462 & 498 & 489 & 675 & 678 & 715 & 719 & 703 & 725 & 741\end{array}$

$\begin{array}{llllllll}726 & 721 & 415 & 305 & 430 & 305 & 745 & 1800\end{array}$

$\begin{array}{lllllllllllllll}3153 & 421 & 457 & 449 & 476 & 469 & 503 & 496 & 675 & 678 & 714 & 718 & 705 & 723 & 737\end{array}$

$\begin{array}{llllllll}726 & 718 & 413 & 305 & 426 & 305 & 737 & 1800\end{array}$

$\begin{array}{lllllllllllllll}3188 & 424 & 459 & 452 & 479 & 471 & 505 & 498 & 675 & 678 & 714 & 718 & 706 & 722 & 736\end{array}$

$\begin{array}{lllllllll}725 & 717 & 413 & 305 & 424 & 305 & 734 & 1800\end{array}$

$\begin{array}{lllllllllllllll}3258 & 429 & 463 & 457 & 483 & 476 & 508 & 503 & 675 & 678 & 713 & 717 & 707 & 721 & 733\end{array}$

$\begin{array}{llllllll}724 & 715 & 412 & 305 & 421 & 305 & 728 & 1800\end{array}$

$\begin{array}{lllllllllllllll}3398 & 439 & 472 & 467 & 491 & 486 & 515 & 512 & 675 & 678 & 711 & 716 & 709 & 719 & 728\end{array}$

$\begin{array}{llllllll}722 & 711 & 410 & 305 & 416 & 305 & 717 & 1800\end{array}$

$\begin{array}{lllllllllllllll}3558 & 449 & 481 & 478 & 499 & 497 & 524 & 521 & 674 & 678 & 711 & 714 & 710 & 716 & 722\end{array}$

$\begin{array}{llllllll}720 & 707 & 408 & 305 & 411 & 305 & 707 & 1800\end{array}$

$\begin{array}{lllllllllllllll}3559 & 449 & 481 & 478 & 499 & 497 & 524 & 521 & 674 & 677 & 711 & 714 & 710 & 716 & 722\end{array}$

$\begin{array}{llllllll}720 & 707 & 408 & 305 & 411 & 305 & 707 & 1800\end{array}$

$\begin{array}{lllllllllllllll}3561 & 450 & 482 & 479 & 499 & 497 & 524 & 521 & 674 & 677 & 711 & 714 & 710 & 716 & 722\end{array}$ $\begin{array}{llllllll}720 & 707 & 408 & 305 & 411 & 305 & 707 & 1800\end{array}$ 


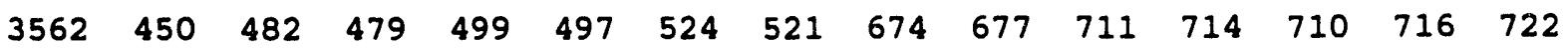
$\begin{array}{llllllll}720 & 707 & 408 & 305 & 411 & 305 & 707 & 1800\end{array}$

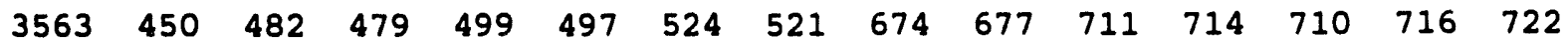

$\begin{array}{llllllll}720 & 707 & 408 & 305 & 411 & 305 & 707 & 1800\end{array}$

$\begin{array}{lllllllllllllll}3564 & 450 & 482 & 479 & 499 & 497 & 524 & 521 & 674 & 677 & 711 & 714 & 710 & 716 & 722\end{array}$

$\begin{array}{llllllll}720 & 707 & 408 & 305 & 411 & 305 & 707 & 1800\end{array}$

$\begin{array}{lllllllllllllll}3565 & 450 & 482 & 479 & 499 & 497 & 524 & 522 & 674 & 677 & 711 & 714 & 710 & 716 & 722\end{array}$ $\begin{array}{llllllll}720 & 707 & 408 & 305 & 411 & 305 & 707 & 1800\end{array}$

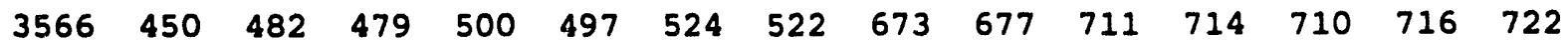

$\begin{array}{llllllll}720 & 707 & 408 & 305 & 411 & 305 & 707 & 1800\end{array}$ 


\section{Source Code Listing}

Following immediately is the main souce code for MELTER. Include files

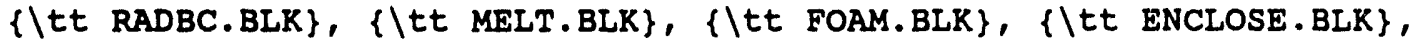
and $\{\backslash$ tt CARGO.BLK\} follow.

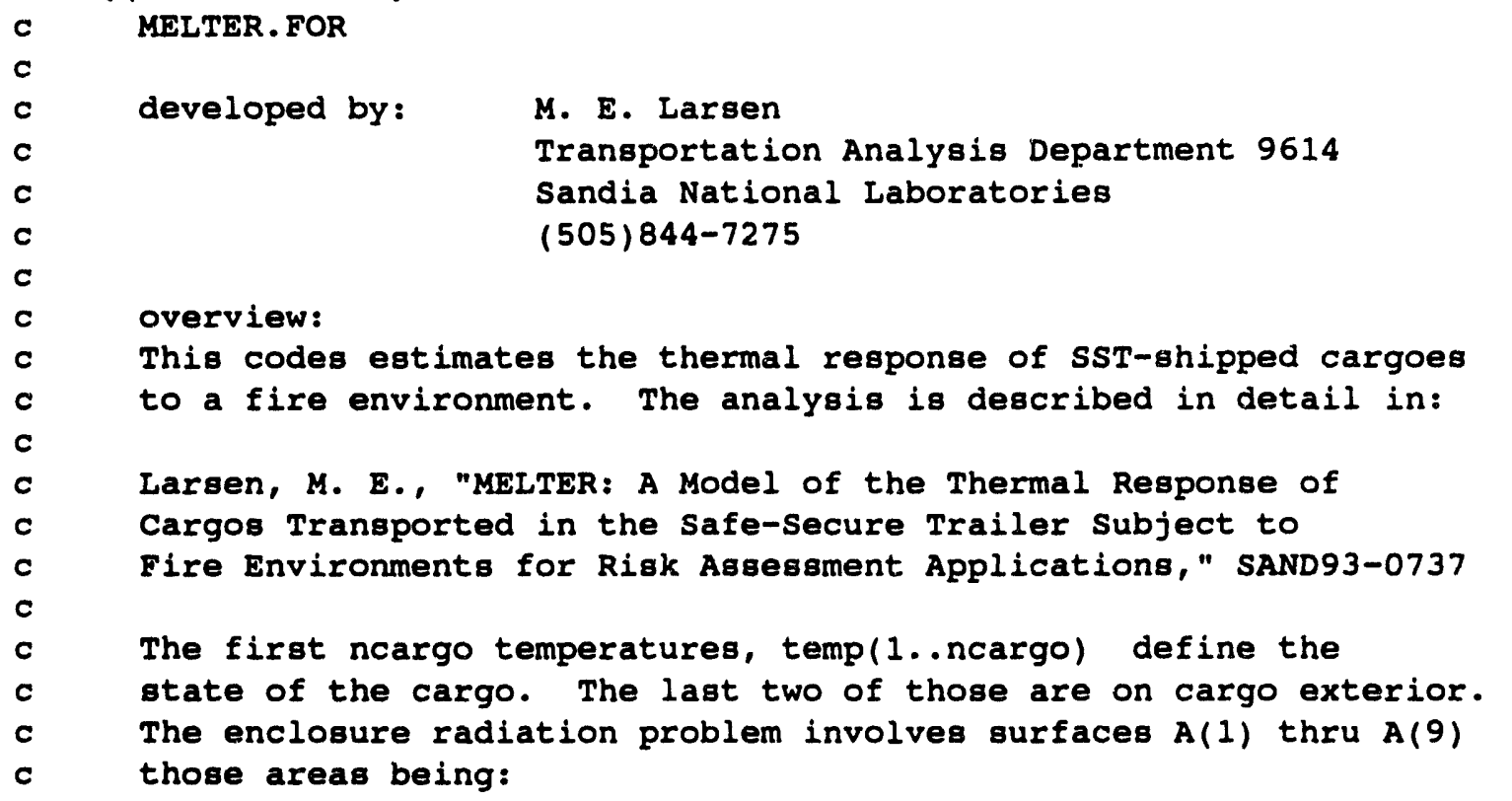

Radiation Area

A(1): hot side of cargo exterior............temp (ncargo-1)

$A(2)$ : cold side of cargo exterior...........temp(ncargo)

$A(3)$ : inert cargo exterior surface...........temp(ncargo+1)

$A(4)$ : floor surf not heated from exterior....temp (ncargo+2)

$A(5)$ : floor surf heated from exterior........temp(ncargot3)

plywood/foam interface..............temp (ncargo+11)

char displacement..................temp (ncargo+10)

exterior skin temperature...........temp (ncargo+9)

$A(6)$ : armor not heated from exterior........temp (ncargo+4)

$A(7)$ : armor heated through intact wall.......temp(ncargo+5)

char displacement.................temp (ncargo+8)

exterior skin temperature............temp (ncargo+7)

$A(8)$ : heated armor with foam stripped off....temp (ncargo+6)

$A(9)$ : hole in armor.................... no temp variable

When modelnumber=1, a melting case, temp(ncargo-1) is temperature until melting begins, thereafter it is really tmelt + $q^{\prime \prime} /\left(\right.$ rho $h C p$ ) where $q^{\prime \prime}$ is the integrated heat flux after melting begins.

the temperature nodes in the "spherized" cargo number up from the inside with the hot nodes having even numbers and cold the odd numbers. If notrip communicated to the code is greater than zero the outer shell is is dismissed and two corresponding variables are removed from the system.
A 1

A 2

A 3

A 4

A 5

A 6

A 7

A 8

A 9

A 10

A 11

A 12

A 13

A 14

A 15

A 16

A 17

A 18

A 19

A 20

A 21

A 22

A 23

A 24

A 25

A 26

A 27

A 28

A 29

A 30

A 31

A 32

A 33

A 34

A 35

A 36

A 37

A 38

A 39

A 40

A 41

A 42

A 43

A 44

A 45

A 46

A 47

A 48 
external funct

character fname*35

character 40 bfile, ofile,tfile, afile, sfile, xfile

A 51

character*24 fdate, starttime, stoptime

A 52

include 'enclose.blk'

include 'radbc.blk'

include 'cargo.blk'

include 'melt.blk'

include 'foam.blk'

A 53

A 54

A 55

A 56

A 57

real terrel, terrmax, emax, hmin, $h$, maxstep, minstep, waymax, hottemp

common /compute/terrel, terrmax, emax, hmin, h, maxstep, minstep, waymax

A 59

real dtimes, dtemps

integer ndtim, ipd, ipdo

common /death/dtimes (20), dtemps (20), ndtim

integer lleft(2), flags(80)

real s8tho2, s8tw, ssth, s8tl

common /s8tdim/ sstho2, s8tw, ssth, sstl

real prox (4), adam9 (6), adam8 (6), ftemp (11), dcdia (10), dedist (7)

real $t f d(30), t t l(30), t e m p(30), \operatorname{dtdt}(30)$

real rtdis(7), rtdia(10), rttem(11), rta(6), rtdam(4)

real work (750)

real relerro, abserro,tpmin, printt, tduro, carga0, cepso, dia

real dist, dtcritdt 1 , tmax, tdur, time, thigh, tlow, fireduration

real timestep, abserr, relerr, $t$ imeold, $t$ ime2, d2tcritdt, tcrit

real tbest, dtboomdt, $t a v$, fuel, tboom

integer nndam, $i$, nadam 9 , ntemp, ndcdia, ncso, noutput, ncrito, icase

integer ipre, idam, iadam, itemp, idia, idist, neq, iflag, lode, Il

integer ia, ndcdist, iflago, maxcalls

integer ndam (4)

integer idiedis(7), idiedia (10), idietem(11), idiea (6), idiedam(4)

integer nrdis(7), nrdia(10), nrtem(11), nra (6), nrdam(4)

logical questl, needtlsolution, needfdsolution

logical kaboom, timeout, doneheating, stillburning

logical boomonce, preempt, passone

set up the data needed for dave's many cases

data idiedis, idiedia, idietem, idiea, idiedam $/ 38 \star 0 /$

data nrdis, nrdia, nrtem, nra, nrdam $/ 38 * 0 /$

data rtdis, rtdia, rttem, rta, rtdam $/ 38 * 0.0 /$

data prox, ndam $/ 3 * 1.0,0.0,3,2,1,0 /$

data adam $9 / 20.0,5.0,0.0,0.0,0.0,0.0 /$

data adam $8 / 40.0,40.0,40.0,20.0,5.0,0.0 /$

data ftemp/3000.0,2600.0,2400.0,2300.0,2200.0,2100.0,2000.0, $\times 1900.0,1800.0,1700.0,1600.0 /$

data dedia/100.0,50.0,40.0,35.0,30.0,25.0,20.0,15.0,10.0,5.0/

data dedist/ $-1.0,0.0,5.0,10.0,20.0,40.0,80.0 /$

data tpmin,printt $/ 2 * 20.0 /$

data relerro, abserro/1.0e-4,0.001/

c maxcalls is the number of times we'll call ode to get by a

c rough spot before punting.

data maxcalls/2/

c terror is the allowed interval of uncertainty in the

c bisection search. It does not make sense for terror to be
A 60

A 61

A 62

A 63

A 64

A 65

A 66

A 67

A 68

A 69

A 70

A 71

A 72

A 73

A 74

A 75

A 76

A 77

A 78

A 79

A 80

A 81

A 82

A 83

A 84

A 85

A 86

A 87

A 88

A 89

A 90

A 91

A 92

A 93

A 94

A 95

A 96

A 97

A 98

A 99

A 100

A 101

A 102 
less than at least 30 seconds, since it can be costly to close

A 103

corresponds to a fire that "nicks" the SST. A negative value

A 106

A 107

A 108

A 109

A 110

A 111

A 112 of distance is used to specify a fire centered under the SST.

A 113

A 114

call getarg( 1 , fname $)$

A 115

bfile=fname (: (index (fname, ' ')-1))//'.brc'

A 116

open (unit $=7$, file=bfile, status='old')

A 117

A 118

c

there has to be the same number of proximities and

A 119

c

damage states considered (4 maximum)

A 120

$\operatorname{read}(7, *)$ nndam, (ndam $(i), i=1, n n d a m)$

A 121

$\operatorname{read}(7, *) \quad(\operatorname{prox}(i), i=1, n$ nndam)

there are the same number of adam9 and adam8 values ( $6 \max$ )

A 122

$\operatorname{read}(7, *) \operatorname{nadam} 9,(\operatorname{adam} 9(i), i=1, \operatorname{nadam} 9)$

A 123

$\operatorname{read}(7, *)(\operatorname{adam} 8(i), i=1, \operatorname{nadam} 9)$

there may be as many as 10 temperatures

A 124

A 125

$\operatorname{read}(7, *)$ ntemp, (ftemp ( $i), i=1$, ntemp)

A 126

A 127

A 128

$\operatorname{read}(7, *)$ ndcdia, (dcdia ( $i), i=1$, ndcdia)

A 129

A 130

there may be as many as 7 distances

A 131

$\operatorname{read}(7, *)$ ndcdist, (dcdist $(i), i=1, \operatorname{ndcdist})$

c

c

tduro is zero if the calculation is to yield the minimum critical

A 133

$\operatorname{read}(7, *)$ tduro

A 134

cloge (7)

c

get input file name here

A 135

A 136

call getarg $(2$, fname $)$

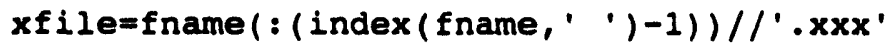

A 137

tfile=fname (: (index (fname, ' )-1))//' tar'

A 138

afile=fname (: (index (fname, ' )-1))//' .mel'

A 139

ofile=fname (: (index (fname, ' ')-1))//' .dat'

A 140

sfile=fname (: (index (fname, ' ')-1))//'.sta'

A 141

open (unit $=4$, file=tfile, status='old' )

A 142

A 143

open (unit $=4$, file='targ.dat', status='old')

A 144

$\operatorname{read}\left(4,{ }^{*}\right)$ ncs 0,1 left $(1), 1$ left $(2)$, modelnumber, noutput

A 145

$\operatorname{read}(4, *)$ cargao, cepso, transm, ttrans, a (3), rmcp3

A 146

read $(4, *)$ tmelt, umelt

A 147

$\operatorname{read}(4, *)$ ncrit 0, ndtim

A 148

do $5 i=1$, ndtim

A 149

read $(4, *)$ dtimes (i), dtemps (i)

A 150

5

continue

$\operatorname{read}(4, *)(\mathrm{ck}(i), i=1, \operatorname{ncs} 0-1)$

$\operatorname{read}(4, *)(\operatorname{cr}(i), i=1, \operatorname{ncs} 0-1)$

A 151

$\operatorname{read}(4, *)(\operatorname{mcp}(i), i=1, \operatorname{ncs} 0)$

A 152

A 153

$\operatorname{read}\left(4,{ }^{*}\right)(\operatorname{rcarg}(i), i=1, \operatorname{ncs} 0)$

A 154

close (4)

A 155

A $\mathbf{1 5 . 6}$ 
open (unit $=8$, file=afile, status= ' unknown' )

open (unit $=17, f i l e=x f i l e$, status= ' unknown')

A 158

rewind (8)

rewind (17)

A 159

icase $=0$

A 160

ipre $=0$

A 161

ipre=0

starttime=fdate ()

A 162

do 666 idam=1, nndam

A 163

proximity $=$ prox (idam)

A 164

nstrip=ndam (idam)

A 165

do 666 iadam=1, nadam9

A 166

$a(8)=\operatorname{adam} 8($ iadam $) * 0.3048 * * 2$

A 167

$a(9)=\operatorname{adam} 9($ iadam $) \star 0.3048 * \star 2$

A 168

do 666 itemp=1, ntemp

A 169

tfire $=($ ftemp $($ itemp $)+460.0) * 5.0 / 9.0$

A 170

do 666 idia $=1$, ndcdia

A 171

dia=dcdia ( idia) $* 0.3048$

A 172

do 666 idist $=1$, ndcdist

A 173

if (dcdist(idist).1t.0.0)then

A 174

dist $=0.0$

A 175

else

A 176

$\operatorname{dist}=(\operatorname{dcdist}($ idist $)+\operatorname{dcdia}($ idia $) / 2.0) * 0.3048+88 t w / 2.0$

A 177

A 178

end if

A 179

dtcritdt $1=0.0$

A 180

$t \max =50000.0$

A 181

passone= . true.

A 182

tdur=tduro

A 183

icase $=i c a s e+1$

A 184

print *, icase, idam, iadam, itemp, idia, idist

A 185

nrdis (idist) $=$ nrdis (idist) +1

A 186

nrdia (idia) $=$ nrdia (idia) +1

A 187

nrtem ( itemp) $=$ nrtem $($ itemp) +1

A 188

nra ( iadam) $=\mathrm{nra}$ ( iadam) +1

$\operatorname{nrdam}($ idam) $=$ nrdam (idam) +1

A 189

if (noutput.gt.o) then

A 190

A 191

print *,'dist',dist, 'dia',dia, 'tfire',tfire, 'a(9)', a(9)

A 192

print *, 'a(8)',a(8), 'nstrip' , notrip, 'proximity' , proximity

A 193

print *,'case ', icase,' running . . . .'

A 194

end if

for the present let the floor fire temp = tfire

A 195

The variables read from bufferl are:

A 196

A 197

A 198

$a(8)-a(9)$ : areas of scraped, and removed surface $\left(m^{-2}\right)$

A 199

dist: separation distance of the fire

A 200

dia: diameter of the fire

A 201

tdur: duration of fire (calculated if tdur.le.0)

A 202

that is, question 2 is imposed if tdur.le.0 else question 1 A 203

tfire: fire temperature (K)

tmax: maximum amount of time allowed for failure (B)

A 204

model number: selects model options as follows:

A 205

A 206

0 full onion, no melting

A 207

1 full onion, skin melts

A 208

A 209

read $\left(1,{ }^{*}\right)$ nstrip, proximity, a $(9), a(8)$, tfire, dia, dist, tdur, tmax

A 210 
The variables read from target. dat are:

ncs0: number of concentric shells representing cargo when undamaged

A 213

A 214

A 215

nstrip: number of layers stripped off

A 216

noutput: parameter determining how much output program generates

A 217

acargo: exterior surface area of target $\left(\mathrm{m}^{-2} 2\right)$

A 218

cargoeps: emisgivity of target surface

transm: tranmissivity of view across SST

ttrans: tranmissivity of view from target to hot wall

A 219

A 220

proximity: $(0-1)$ gives proximity of target to wall, 1 is

A 221

A 222 next to wall

a(3): surface area of inerts (make arbitrarily large) (m²)

A 223

rmcp3: thermal capacitance of inert cargo $(\mathrm{J} / \mathrm{K})$

ncrit: number of shell to be watched for critical condition

A 224

tcritical: critical temperature (K)

ck(i): conduction connector between $\operatorname{mcp}(i) \& \operatorname{mcp}(i+1)(\mathrm{w} / \mathrm{k})$

A 225

A 226

A 227

cr(i): radiation connector between $\operatorname{mcp}(i)$ and $\operatorname{mcp}(i+1)(W / E)$

A 228

( $E$ being emissive power)

mcp(i): thermal capacitance of ith shell (J/K)

rcarg(i): radii of mcp(i)

tmelt: melting temperature of $\operatorname{mcp}(\mathrm{ncs} 0)$ (K)

A 229

A 230

A 231

A 232

umelt: (latent heat of fusion)/Cp

A 233

A 234

makemod develops the necessary radiation matrix and sets other

A 235 constants used in subroutine funct to evaluate the time

A 236

rate of change of temperatures

A 237

A 238

A 239

tfiref $=t$ fire

canmelt $=$. false.

A 240

A 241

A 242

A 243

A 244

A 245

A 246

A 247

A 248

A 249

A 250

A 251

A 252

ok to melt

if (noutput.gt.0) then

A 253

open (unit=11, file=of ile, status= ' unknown' )

A 254

rewind (11)

end if

quest $1=($ tdur. gt . 0.0$)$

A 255

A 256

A 257

call makemod(neq, dist, dia, preempt, noutput, ncso, ncrito, cepso,

A 258

1carga0)

if (preempt) then

A 259

A 260

kaboom $=$, false.

A 261

boomonce= false.

A 262

timeout $=$. true.

A 263

$t$ ime $=0.0$

A 264 
temp (ncrit) $=t$ inf

A 265

thigh $=0.0$

A 266

t low $=0.0$

A 267

ipre=ipre+1

A 268

goto 200

A 269

end if

c write out problem description

A 270

if (noutput.gt.0) then

A 271

write $(11,777)$ starttime

A 272

A 273

777 format(' Melter Run at ',a24,///)

write $(11,778)$ 'The total number of state variables is', neq

A 274

write $(11,778)$ 'The cargos number of concentric shel1 's', ncs

A 275

A 276

write $(11,778)$ 'The cargos number of temperatures', ncargo

A 277

write $(11,779)$ 'The sunny side of the cargo has area',a(1)

A 278

write $(11,779)$ 'The dark side of the cargo has area',a(2)

A 279

write $(11,779)$ 'Inerts have area', a(3)

write $(11,779)$ 'Floor area not directly exposed',a(4)

A 280

write $(11,779)$ 'Floor area directly exposed',a(5)

write $(11,779)$ 'Wall interior not directly exposed', a(6)

write $(11,779)$ 'Normal wall directly exposed', a(7)

write $(11,779)$ 'Armor with foam removed', a(8)

write $(11,779)$ 'Hole area', a(9)

write $(11,779)$ 'Distance from the fire is', dist

write $(11,779)$ 'The fire diameter (m) is', dia

write $(21,779)$ 'The view of $a(7 \ldots 9)$ to fire is', f7f

if (quest 1) then

write $(11,779)$ 'The specified fire duration is',tdur

A 281

A 282

A 283

A 284

A 285

A 286

A 287

A 288

A 289

A 290

else

A 291

A 292

write $(11,779)$ 'The fire duration will be calculated'

A 293

end if

write $(11, *)$ ' Output is ordered: '

A 294

write $\left(11,{ }^{*}\right)$ ' time, $T(1) \ldots T$ (ncargo), $T(A 3) \ldots T(A 8), T$ gkin(A7),

A 295

$1 \times \operatorname{char}(A 7), T a k i n(A 5), \operatorname{xchar}(A 5) \cdot$

A 296

A 297

A 298

778 format $(a 40,112)$

779 format $(a 40, f 12.3)$

A 299

780 format (a78)

end if

do $20 i=1$, neq

$20 \operatorname{temp}(i)=\operatorname{tinf}$

if $a(7)>0$ then temp(char7id) is char displacement in wall

if $(\operatorname{ahere}(7))$ temp $(\operatorname{char} 7 i d)=0.0$

c

if $a(5)>0$ then temp(char5id) is char displacement in floor

if (ahere(5)) then

$\operatorname{temp}(\operatorname{char} 5 i d)=0.0$

temp (plyid)=tinf

end if

time $=0$.

A 300

A 301

A 302

A 303

A 304

A 305

A 306

A 307

A 308

A 309

A 310

A 311

stillburning=.true.

A 312

doneheating=. false.

A 313

kaboom= . false.

boomonce= false.

needtlsolution=. true.

A 314

A 315

A 316

needfdsolution=. true.

A 317

C

set initial interval of uncertainty for minimum fire duration

A 318 


\begin{tabular}{|c|c|c|}
\hline & ipdo $=-1$ & A 319 \\
\hline & tlow $=0.0$ & A 320 \\
\hline & thigh $=40.0 * 15000.0 * 60.0 /(3.141592 *(\mathrm{dia} / 0.3048) \star \star 2)$ & A 321 \\
\hline & if (thigh.gt.tmax/1.1)tmax=thigh t. .1 & A 322 \\
\hline C & maximum possible fire duration in seconds & A 323 \\
\hline C & starting point for the bisection method loop & A 324 \\
\hline 84 & continue & A 325 \\
\hline & printt $=t$ low & A 326 \\
\hline & stillburning =.true. & A 327 \\
\hline & doneheating=. false. & A 328 \\
\hline & kaboom=. false. & A 329 \\
\hline & efire $=8$ igma $* t$ fire $* \star 4$ & A 330 \\
\hline & efiref $=8$ igma $* t$ firef $* \star 4$ & A 331 \\
\hline & ehole=einf*(1.0-f9f)+f9f*efire & A 332 \\
\hline & if (quest1) then & A 333 \\
\hline & fireduration=tdur & A 334 \\
\hline & else & A 335 \\
\hline c & bisect the region of uncertainty & A 336 \\
\hline & if (passone) then & A 337 \\
\hline & fireduration=thigh & A 338 \\
\hline & else & A 339 \\
\hline & fireduration $=($ thigh + tlow $) / 2.0$ & A 340 \\
\hline & end if & A 341 \\
\hline & end if & A 342 \\
\hline $\mathbf{c}$ & atarting point for integration (commences at tlow) & A 343 \\
\hline c & always start slow & A 344 \\
\hline & timestep=minstep & A 345 \\
\hline & abserr=abserro & A 346 \\
\hline & relerr $=$ relerro & A 347 \\
\hline & iflag=1 & A 348 \\
\hline 92 & continue & A 349 \\
\hline & timeold=time & A 350 \\
\hline & time2 =time+t imestep & A 351 \\
\hline & if $((t$ ime2.gt.tlow).and.(time.1t.tlow $))$ then & A 352 \\
\hline c & force the end of an integration step to land on & A 353 \\
\hline & time2 =t low & A 354 \\
\hline & iflago $=-1$ & A 355 \\
\hline & else if ((time2.gt.fireduration).and.(time.lt.fireduration))then & A 356 \\
\hline C & force the end of an integration step to land on fireduration & A 357 \\
\hline $\mathbf{C}$ & the exact moment the fire goes out & A 358 \\
\hline & time2=fireduration & A 359 \\
\hline & iflago $=-1$ & A 360 \\
\hline & else if ((time2.gt.thigh).and.(time.1t.thigh)) then & A 361 \\
\hline C & force the end of an integration step to land on thigh & A 362 \\
\hline & time2=thigh & A 363 \\
\hline & iflago $=-1$ & A 364 \\
\hline & else & A 365 \\
\hline & iflago $=1$ & A 366 \\
\hline & end if & A 367 \\
\hline & if (time.ge.tlow) then & A 368 \\
\hline & if (needtlsolution) then & A 369 \\
\hline & needtlsolution=.false. & A 370 \\
\hline C & $\begin{array}{l}\text { save state at tlow in case that is the point at which we commence } \\
\text { do } 40 i=1 \text {, neq }\end{array}$ & $\begin{array}{l}\text { A } 371 \\
\text { A } 372\end{array}$ \\
\hline
\end{tabular}




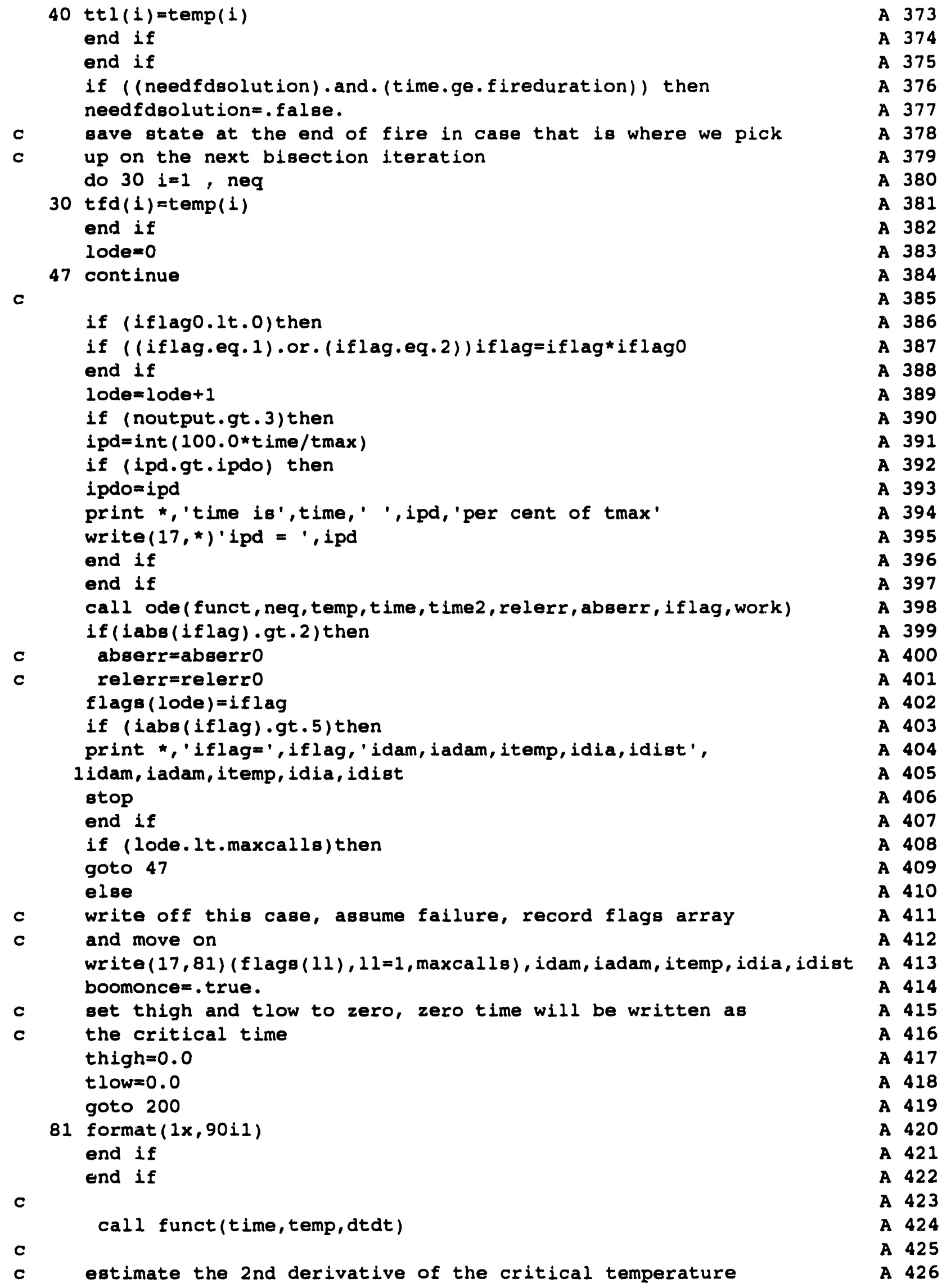

estimate the 2 nd derivative of the critical temperature 
$d 2 t \operatorname{critd}=(d t d t$ (ncrit) $-d t \operatorname{critdt} 1) /(t i m e-t i m e o l d)$

A 428

dtcritdt $1=d t d t($ ncrit)

A 429

tcrit $=$ tboom ( $t$ ime)

A 430

C

new timestep management as of $5 / 28 / 93$

A 431

timestep managed as follows:

A 432

A 433

A 434

$c$

c

while fire is on

A 435

timestep=amax 1 (minstep, terrel*aminl (fireduration, time)/2.0)

A 436

A 437

after fire is out timestep=maxstep

A 438

note that prior to ode call assurances are made to adjust timestep $A 439$

so that an integration step ends on tlow and/or on fireduration. $A 440$

if (atiliburning) then

A 441

if (time.ge.fireduration) then

A 442

extinguish fire

A 443

ehole=einf

A 444

efiremeinf

A 445

efirefmeinf

A 446

stillburning $=$. false.

A 447

end if

A 448

timestep=amax 1 (mingtep, terrel*amin1 (fireduration, time) $/ 20.0$ )

A 449

c

the previous line apparently allows huge steps capping timestep

A 450

if (timestep.gt.maxstep) $t$ imestep=maxstep

else

timestep=maxatep

end if

$t$ imeout $=$ (time.gt.tmax)

if (timeout.and.stillburning) goto 200

kaboom= (temp (ncrit).gt.tboom(time))

boomonce= (kaboom . or . boomonce)

if (time.gt.fireduration) then

C

c

"doneheating" is true if the fire is out and Tcrit declines

faster than tboom

dtboomdt $=$ tboom $(t$ ime +1.0$)-t c r i t$

doneheating=(dtdt (ncrit). $1 t . d t b o o m d t)$

C

c

c

c

c

c

C

c

by this point the 2nd derivative of tcrit should be negative

for the rest of the solution. If it is, and at our current

rate of temperature climb we won't make it to failure, we can

bail out.

if ( (d2tcritdt.1t.0.0) and. (time.gt.3.0*fireduration) ) then

tbest $=(t$ boom $(t \max )-t e m p(n c r i t)) / \operatorname{dtdt}$ (ncrit)

doneheating $=(t$ best.gt. $\cdot($ tmax-time $))$

end if

at this point the fire is out. if no temperature in the system exceeds tboom(tmax) there is no possibility of making it

to failure

A 451

A 452

A 453

A 454

A 455

A 456

A 457

A 458

A 459

A 460

A 461

A 462

A 463

A 464

A 465

A 466

A 467

A 468

A 469

A 470

A 471

A 472

A 473

A 474

hottemp $=0.0$

A 475

A 476

do $71 i=1$, neq

hottemp $=\operatorname{amax} 1$ (temp (i), hottemp)

A 477

A 478

71 continue

if (hottemp. It. tboom ( $t \max )$ ) doneheat ing=. true.

A 479

A 480 


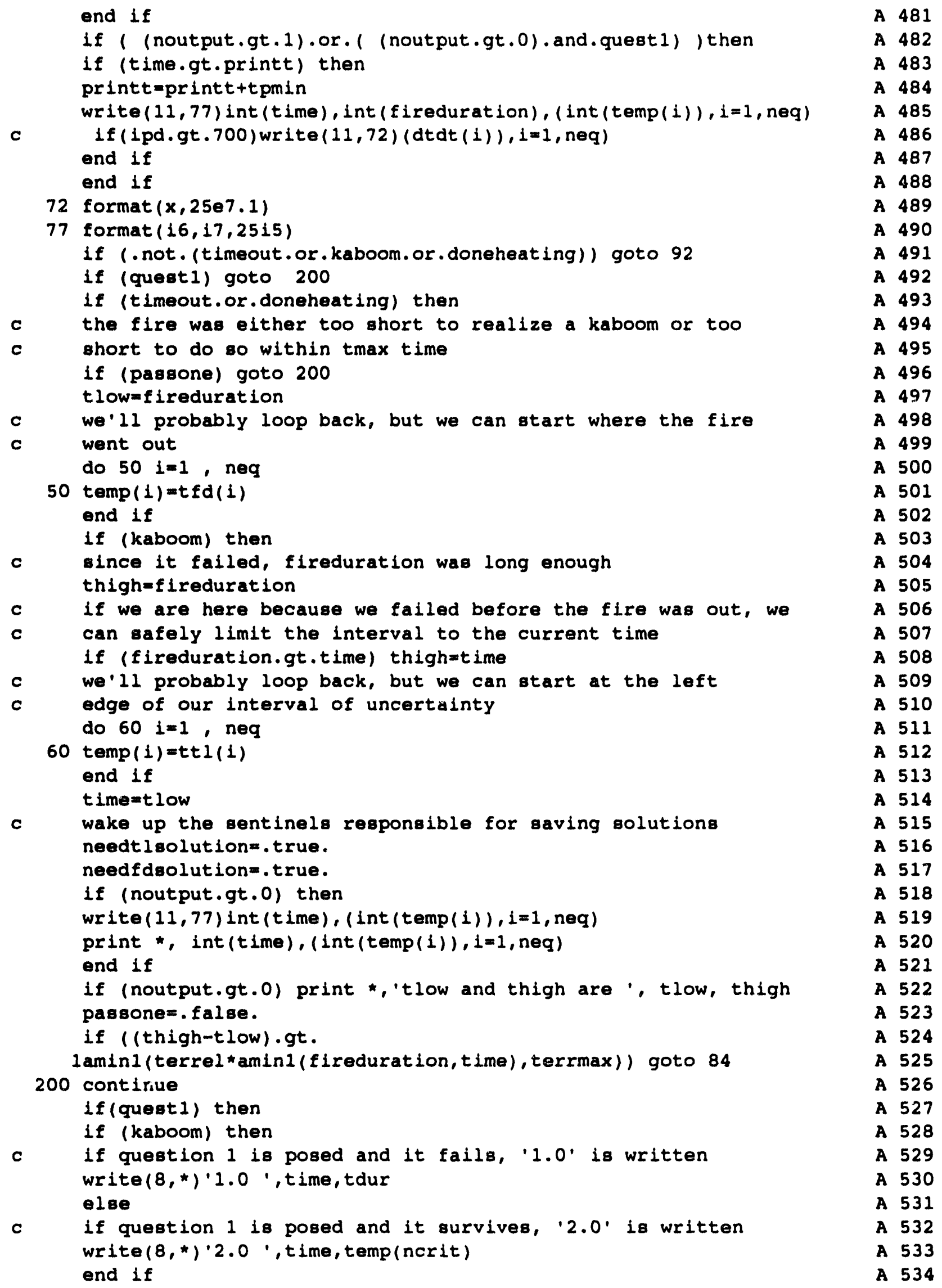

A 532

A 533

A 534 


\begin{tabular}{|c|c|c|c|}
\hline \multirow{3}{*}{ c } & else & A & 535 \\
\hline & question 2 has been asked & A & 536 \\
\hline & if (.not.boomonce) then & A & 537 \\
\hline c & If question 2 is posed and no failure is found within tmax, ' $2.0^{\prime}$ & A & 538 \\
\hline C & write $(8, *) \cdot 2.0 ', t \max , t e m p(n c r i t)$ & A & 539 \\
\hline & write $(8,79)$ idam, iadam, itemp, idia, idist & A & 540 \\
\hline 79 & format $(1 x, 1.0071 .007 \cdot, 513)$ & A & 541 \\
\hline & If (lode.gt.1) then & A & 542 \\
\hline & write $(17,82)$ lode, relerr, abserr, idam, iadam, itemp, idia, idist & A & 543 \\
\hline & end if & A & 544 \\
\hline & elge & A & 545 \\
\hline c & If question 2 is posed and failure is found within tmax, 1.0 ' & $\mathbf{A}$ & 546 \\
\hline c & thigh and tlow bound the minimum fire duration leading to failure & A & 547 \\
\hline c & write $(8, *) \cdot 1.0 \cdot$, thigh, tlow & A & 548 \\
\hline & $t a v=(t h i g h+t l o w) / 2.0$ & A & 549 \\
\hline \multirow[t]{23}{*}{ c } & compute the critical quantity of fuel corresponding to tav & A & 550 \\
\hline & $f u e l=\operatorname{tav} /(40.0 * 60.0 /(3.141592 *(\mathrm{dia} / 0.3048) * * 2))$ & A & 551 \\
\hline & write $(8,78)$ tav, fuel, idam, iadam, itemp, idia, idist & A & 552 \\
\hline & format $(1 \times, 2 f 10.1,513)$ & A & 553 \\
\hline & if (lode.gt. I) then & A & 554 \\
\hline & write $(17,82)$ lode, relerr, abserr, idam, ladam, itemp, idia, idist & A & 555 \\
\hline & end if & $\mathbf{A}$ & 556 \\
\hline & format $(1 x, 12,2 e 12,4,5 i 3)$ & A & 557 \\
\hline & idiedis(idist)=idiedis(idist) +1 & A & 558 \\
\hline & idiedia (idia) =idiedia (idia) +1 & A & 559 \\
\hline & idietem (itemp) = idietem (itemp) +1 & A & 560 \\
\hline & Idiea $($ iadam $)=i d i e a($ iadam $)+1$ & $\mathbf{A}$ & 561 \\
\hline & Idiedam ( Idam) = idiedam ( Idam) +1 & $\mathbf{A}$ & 562 \\
\hline & $\operatorname{rtdis}(i d i s t)=r t d i s(i d i s t)+t a v$ & $\mathbf{A}$ & 563 \\
\hline & $\operatorname{rtdia}(i d i a)=r t d i a(i d i a)+t a v$ & $\mathbf{A}$ & 564 \\
\hline & rttem ( itemp) $=$ rttem (itemp) +tav & $\mathbf{A}$ & 565 \\
\hline & rta (iadam) $=r t a($ iadam) +tav & $\mathbf{A}$ & 566 \\
\hline & rtdam (idam) $=r t d a m(i d a m)+t a v$ & A & 567 \\
\hline & and if & A & 568 \\
\hline & end if & $\mathbf{A}$ & 569 \\
\hline & if (noutput.gt.0) close(11) & A & 570 \\
\hline & $\operatorname{call}$ flush $(8)$ & $\mathbf{A}$ & 571 \\
\hline & call flush (17) & A & 572 \\
\hline \multirow[t]{2}{*}{666} & continue & $\mathbf{A}$ & 573 \\
\hline & etopt ime=fdate () & A & 574 \\
\hline \multirow[t]{14}{*}{$c$} & write $(8, *)$ ' this run begun at ', starttime,' ended ', stoptime & $\mathbf{A}$ & 575 \\
\hline & write $(17, *)$ ' this run begun at ', starttime,' ended ', stoptime & A & 576 \\
\hline & close $(8)$ & A & 577 \\
\hline & close (17) & A & 578 \\
\hline & open (unit $=8$, file=sfile, status=' unknown') & A & 579 \\
\hline & rewind (8) & $\mathbf{A}$ & 580 \\
\hline & write $(8,)^{\prime}$ ' this run begun at ', starttime,' ended ', stoptime & A & 581 \\
\hline & write $(8, *)$ 'dist desths',(idiedis(idist), idist $=1$, ndcdist) & A & 582 \\
\hline & write $\left(8,{ }^{*}\right)$ 'dist trials',(nrdis(idist), idist=1,ndcdist) & A & 583 \\
\hline & write $\left(8,{ }^{*}\right)$ 'dist av tim',((rtdis(idist)/nrdis(idist)), & A & 584 \\
\hline & 1 idist $=1$, ndcdist) & A & 585 \\
\hline & write $\left(8,{ }^{*}\right)$ 'dia deaths', (idiedia(idia), idia $=1$ & A & 586 \\
\hline & write $\left.(8,)^{\prime}\right)^{\prime}$ ia trials',(nrdia(idia), idia=l, ndcdia) & A & 587 \\
\hline & write $\left(8,{ }^{*}\right)$ 'dia av tim',((rtdia(idia)/nrdia(idia)), idia=1, ndcd & A & 588 \\
\hline
\end{tabular}




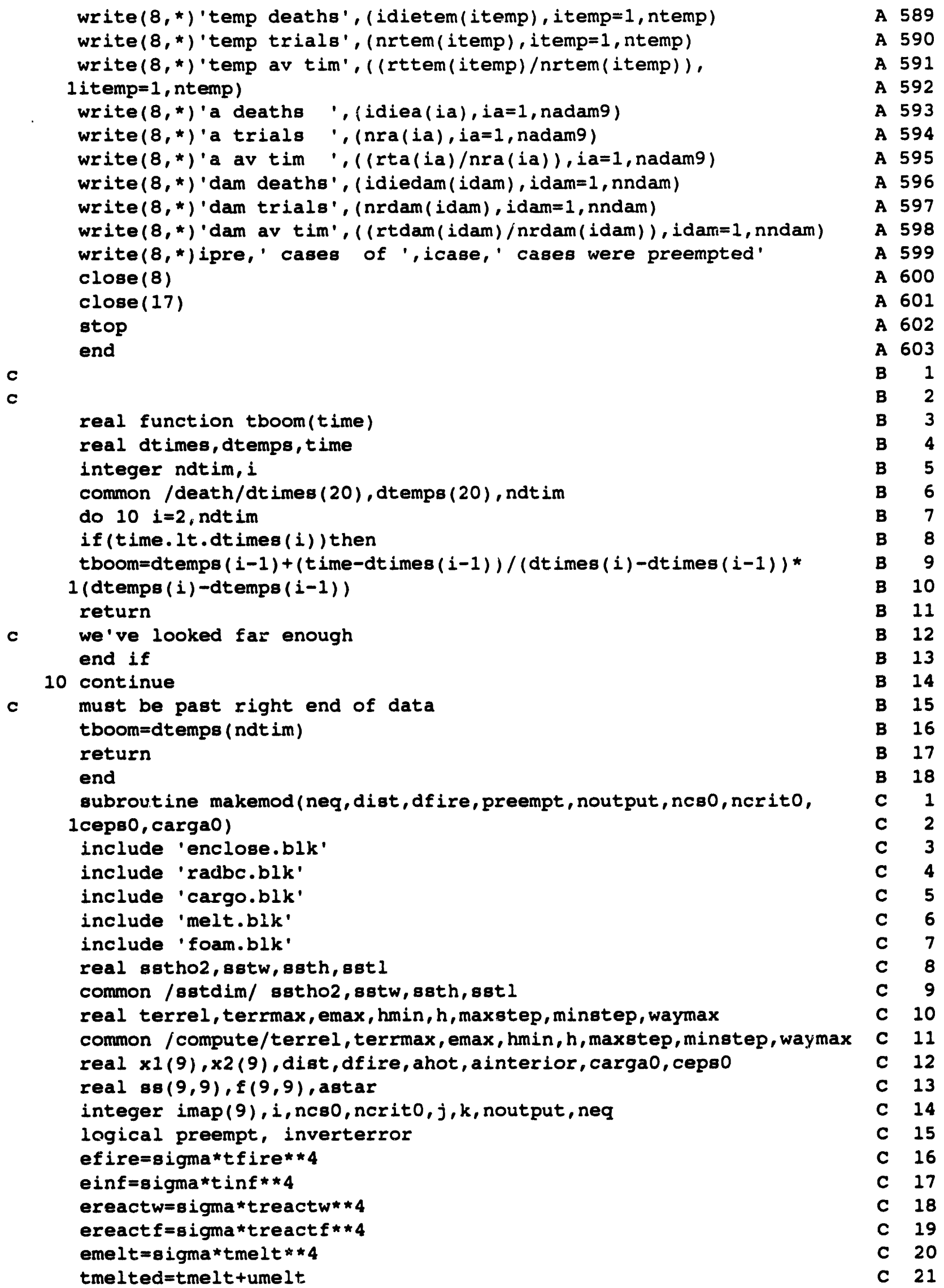


melting=.false. $\quad$ C 22

melted=.false. $\quad$ C 23

cool=.true. $\quad$ C 24

call bc(dist,dfire,treactw,a(5),a(7)) $\quad$ c 25

$f 5 f=1.0 \quad$ C 26

$f 8 f=f 7 f \quad C \quad 27$

$f 9 f=f 7 f \quad C \quad 28$

c $a(9)$ and $a(8)$ snitch from $a(7)$ in that order $\quad$ C 29

if $(a(9) . g t .0 .0) a(7)=\operatorname{amax} 1(0.0, a(7)-a(9)) \quad 30$

if $(a(8) \cdot g t .0 .0) a(7)=\operatorname{amax} 1(0.0, a(7)-a(8)) \quad$ C 31

c a(7) may have vanished, re-assert flag $\quad$ C 32

c add up the area that is heated $\quad$ C 33

$a(4)=88 t 1 * 88 t w-a(5) \quad 34$

$a(6)=88 t l * 88 t w+88 t h * 2.0 *(88 t w+88 t l)-a(7)-a(8)-a(9) \quad$ C 35

ahot $=a(5) \quad$ C 36

do $10 i=7,9 \quad$ C 37

ahot=ahot $+a(i) \quad$ C 38

10 continue

ainterior $=0.0 \quad$ C 40

do $20 i=4,9 \quad$ C 41

ainterior=ainterior $+a(i) \quad$ C 42

20 continue

acargo=cargao

cargoeps $=$ cepso

c write $(2, *)$ ' acargo ',acargo,' ainterior ', ainterior, 'ahot ', ahot

split=ahot/ainterior

$\operatorname{arat}=(\operatorname{rcarg}(n c s-1) / \operatorname{rcarg}(n c s)) \star \star 2$

if (ncs.lt.ncso) then

$\operatorname{acargo}=\operatorname{cargaO} *(\operatorname{rcarg}(n \mathrm{Cs}) / \operatorname{rcarg}(\mathrm{ncs} O)) \star \star 2$

cargoeps $=1.0$

c the exterior of what is left is radiatively black if

c stripping happened.

end if

if (ncrito.gt.1) then

ncrit $=2$ * (ncrit $0-1)$

else

nerit $=$ ncrito

end if

ncargo $=2$ *ncs -1

$a(1)=8 p l i t \star a c a r g o$

c write $(2,)^{\prime} \mathrm{a}(1)$ just after it was set ',a(1)

$a(2)=$ acargo-a (1)

preempt $=($ ahot. . le.0.0)

if (preempt) return

do $30 i=1,9$

ahere $(i)=(a(i) \cdot g t .0 .0)$

30 continue

astar is the largest a surface on the sgt's interior can be

with a zero self view factor

astar $=88 t h * 8 s t l$

$\operatorname{rch}(3)=\operatorname{rmcp} 3 / a(3)$

c

write $(2, \star)^{\prime} \operatorname{rmcp} 3, a(3), \operatorname{rch}(3)^{\prime}, \operatorname{rmcp} 3, a(3), \operatorname{rch}(3)$

eps (1) =cargoeps

eps $(2)$ =cargoeps 


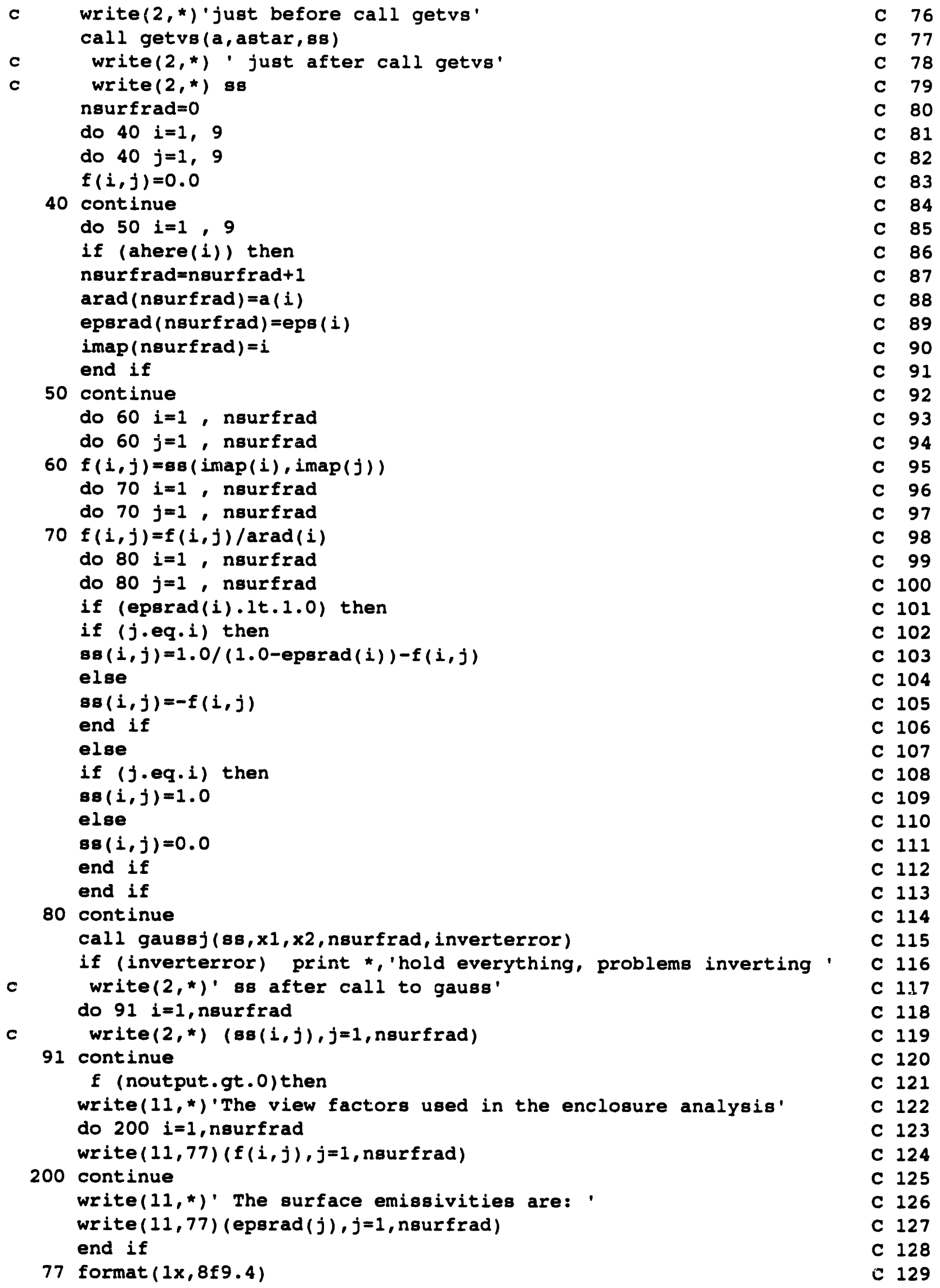


do $100 i=1$, nsurfrad $\quad$ C 130

$100 f(i, i)=f(i, i)-1.0$

C 131

do $110 i=1$, nsurfrad $\quad$ C 132

do $110 j=1$, nourfrad $\quad$ C 133

$\mathrm{hh}(i, j)=0.0 \quad$ C 134

do $130 \mathrm{k}=1$, nsurfrad $\quad$ C 135

$130 h h(i, j)=h h(i, j)+f(i, k) * g 8(k, j) \quad 136$

110 continue $r \begin{gathered}\text { C } 1.37 \\ \end{gathered}$

$j=0$

C 138

$\begin{array}{lll}\text { establish indices between integrated variable and numbered } & C & 139 \\ \text { surfaces and between radiation enclosure model and numbered } & \mathrm{C} 140\end{array}$

surfaces. the array aid holds the equation number corresponding $C 141$

to any numbered surface. the array qid holds the radiation 142

enclosure surface id corresponding to any such surface. isurf C 143

holds the equation number corresponding to a radiation enclosure C 144

surface.

C 145

C 146

aid(i) is the statevariable number of surface $i$, $i$ being 1-9 C 147

invariant gid(i) is the radiation enclosure surface number of 148

invariant $i$ 1-9 isurf $(j)$ is the state variable belonging to 149

enclosure anal. gurf $j$

C 150

C 151

do $140 i=1,9$

C 152

if (ahere(i)) then

$j=j+1$

$\operatorname{aid}(i)=j+$ ncargo-2

C 153

C 154

$\operatorname{qid}(i)=j$

isurf $(j)=a i d(i)$

C 155

C 156

end if

C 157

C 158

C 159

C 160

C 161

C 162

C 163

C 164

C 165

C 166

C 167

C 168

c 169

C 170

C 171

C 172

C 173

c 174

c 175

C 176

C 177

C 178

D 1

oubroutine funct(time, temp,dtdt)

D 2

include 'enclose.blk'

include 'radbc.blk'

include 'cargo.blk'

D 3

include 'melt.blk'

D 4

D 5 


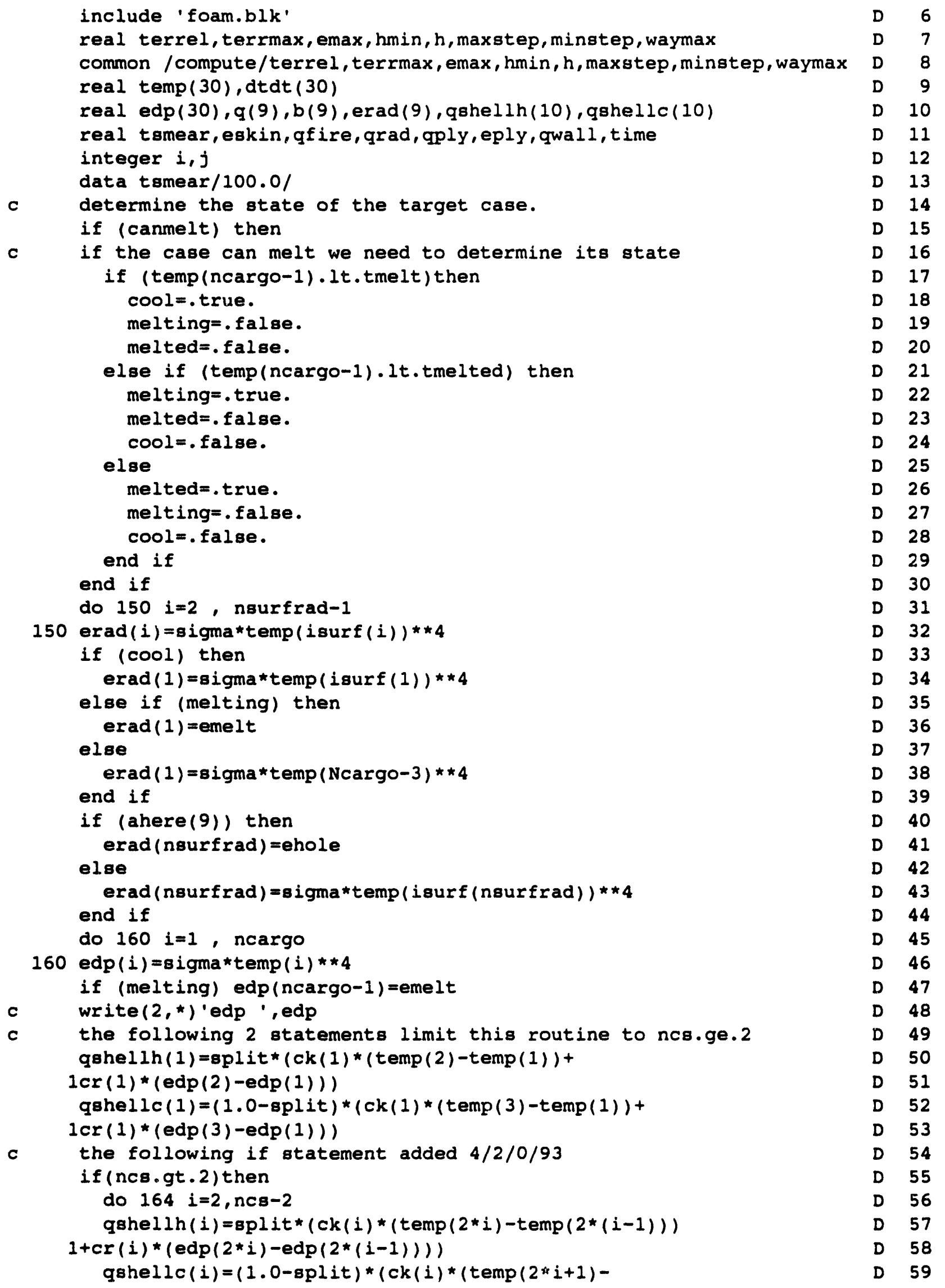




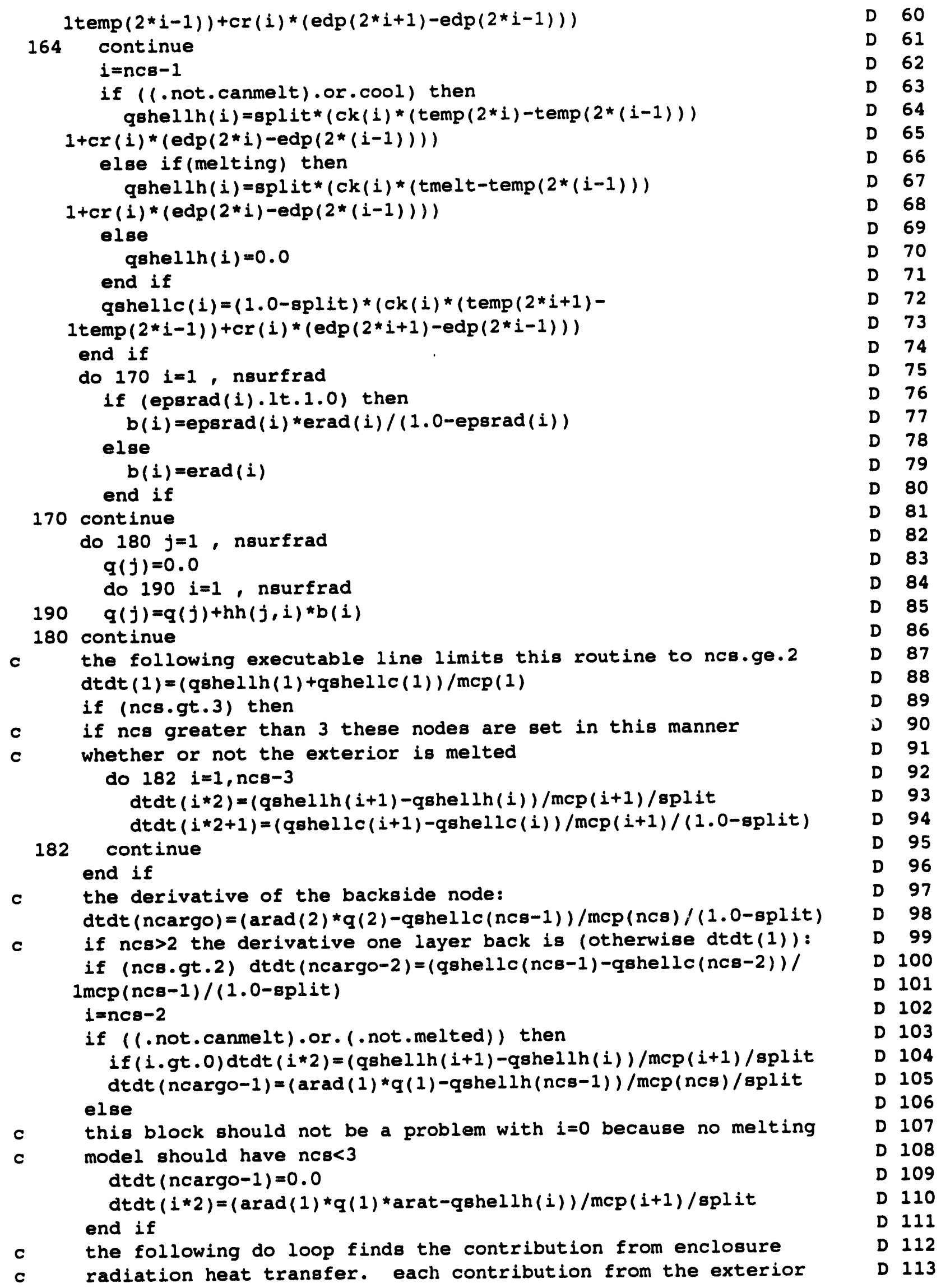


c will be added later.

if $(\operatorname{ahere}(3)) \operatorname{dtdt}(\operatorname{aid}(3))=q(q i d(3)) / \operatorname{rch}(3)$

D 115

if $(\operatorname{ahere}(4)) \operatorname{dtdt}(\operatorname{aid}(4))=q(q i d(4)) / \operatorname{rch}(4)$

D 116

if $(\operatorname{ahere}(6)) \operatorname{dtdt}(\operatorname{aid}(6))=q($ qid $(6)) / \operatorname{rch}(6)$

D 117

if (ahere(5)) then

D 118

c modified to include plywood for christmas

D 119

c

this is the heated part of the floor

eskin=gigma *temp (8kin5id) * 4

c

write $\left.(2,)^{*}\right)$ 'skin5id is 'skin5id

qfire=epso5* (f5f*efiref $+(1.0-f 5 f)$ *einf-eskin)

D 120

D 121

D 122

if (temp(char5id).1t.1floor) then

D 123

if (temp(skin5id).gt.treactf) then

D 124

D 125

qrad $=($ eskin-ereactf $) /(1.0+\operatorname{aafloor} * 0.75 * \operatorname{temp}($ char5id $))$

D 126

else

grad $=0.0$

D 127

D 128

end if

D 129

dtdt (char5id) $=$ qrad / heatreqf

qply $=($ treact $f-t e m p(p l y i d)) /(1$ floor-temp (char5id))

D 130

$1 * k v i r g$

qply=aminl (qply, qrad)

else

$\operatorname{dtdt}(\operatorname{char} 5 i d)=0.0$

eply=gigma temp (plyid) * *4

qply $=(e 8 k i n-e p l y) /(1.0+a a f l o o r * 0.75 * 1$ floor $)$

grad =qply

end if

qwall $=($ temp $($ plyid $)-t e m p(a i d(5))) /$ reply

D 131

D 132

D 133

D 134

D 135

D 136

D 137

D 138

D 139

D 140

dtdt (aid(5)) $=($ qwall $+q(q i d(5))) / \operatorname{rch}(5)$

D 141

dtdt (skin5id) $=$ (qfire-grad)/rchskinf

D 142

dtdt (plyid) $=($ qply-qwall) / rchply

end if

if (ahere(7)) then

eskin=sigma *temp (skin $7 i d) * \star 4$

c

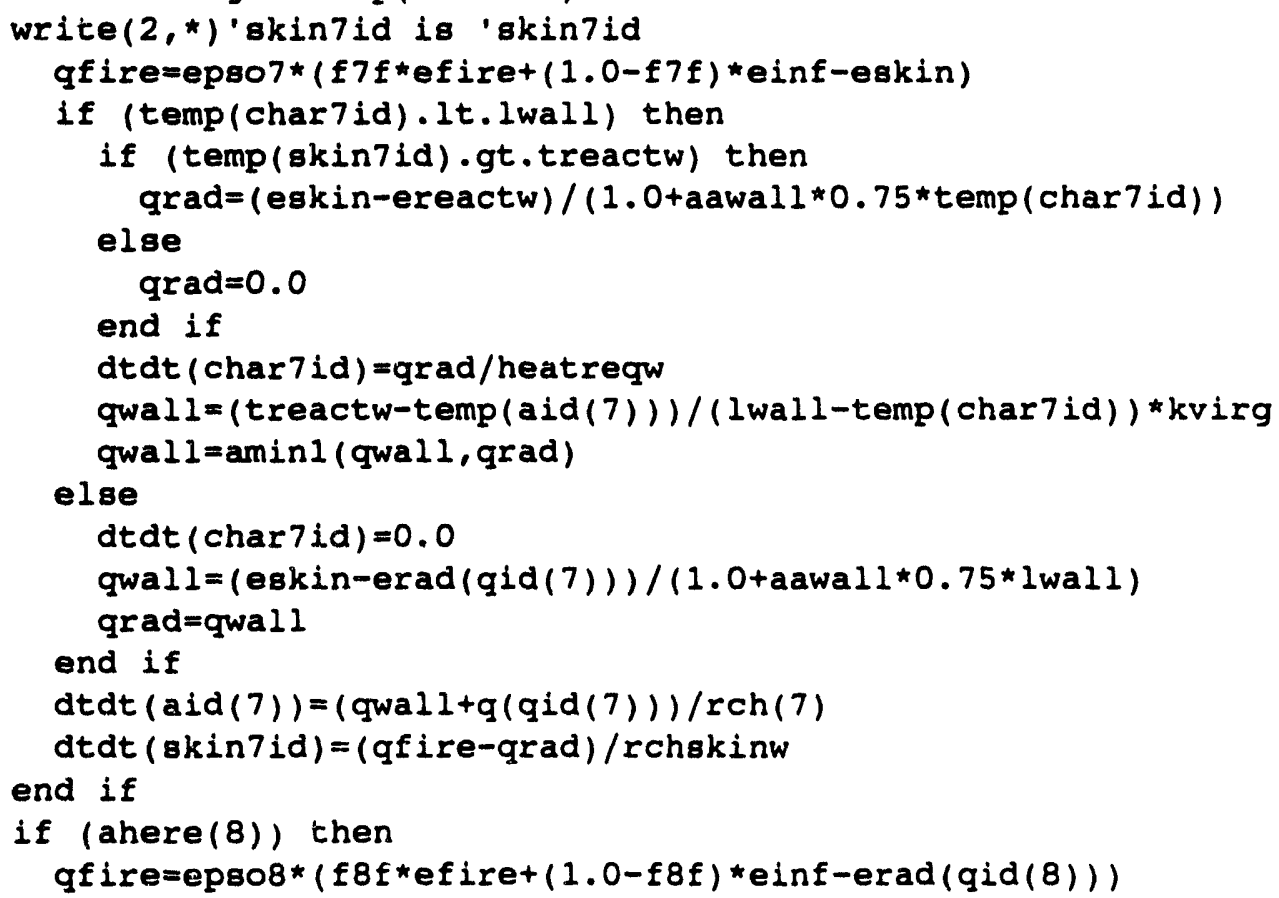

D 143

D 144

D 145

D 146

D 147

D 148

D 149

D 150

D 151

D 152

D 153

D 154

D 155

D 156

D 157

D 158

D 159

D 160

D 161

D 162

D 163

D 164

D 165

D 166

D 167 


\begin{tabular}{|c|c|c|}
\hline & $\operatorname{dtdt}(\operatorname{aid}(8))=(q f i r e+q(q i d(8))) / \operatorname{rch}(8)$ & D 168 \\
\hline & end if & D 169 \\
\hline & return & D 170 \\
\hline & end & D 171 \\
\hline & subroutine getvs(a, astar, $f$ ) & $\mathbf{E}$ \\
\hline c & this procedure calculates all the needed view factors. it starts & $\mathbf{E}$ \\
\hline c & by solving a full 9 by 9 matrix of views. then for cases not & $\mathbf{E}$ \\
\hline c & involving some of the model areas, rows and columns will be & $\mathbf{E}$ \\
\hline c & eliminated. & $\mathbf{E}$ \\
\hline c & & $\mathbf{E}$ \\
\hline & include 'radbc.blk' & $\mathbf{E}$ \\
\hline & include 'cargo.blk' & $\mathbf{E}$ \\
\hline & include 'melt.blk' & $\mathbf{E}$ \\
\hline & real a $(9), f(9,9), a x(9), t \operatorname{sum}(9)$, astar, seered & 10 \\
\hline & integer $i, j$ & 11 \\
\hline & do $10 i=1,9$ & 12 \\
\hline & do $10 j=1,9$ & 13 \\
\hline 10 & $f(i, j)=0.0$ & 14 \\
\hline & do $20 i=1,6$ & 15 \\
\hline 20 & $a x(i)=a(i+3)$ & 16 \\
\hline c & & 17 \\
\hline c & get6v estimates viewfactors between the surfaces that make & 18 \\
\hline c & up the interior wall area of the SST. The view factors that & 19 \\
\hline c & will be returned in $f$ are not yet attenuated by "transm" & 20 \\
\hline c & & 21 \\
\hline & call get $6 v(a x, a s t a r, f)$ & 22 \\
\hline c & unattenuated views between enclosure walls are now in the upper & 23 \\
\hline C & left corner of the f-matrix. move to lower right corner. & 24 \\
\hline & do $30 i=9,4,-1$ & 25 \\
\hline & do $30 j=9,4,-1$ & 26 \\
\hline 30 & $f(i, j)=f(i-3, j-3)$ & 27 \\
\hline c & $a(1)$ and (2) can't see themselves or each other & 28 \\
\hline & do $40 i=1,2$ & 29 \\
\hline & do $40 j=1,2$ & 30 \\
\hline 40 & $f(i, j)=0.0$ & 31 \\
\hline c & & 32 \\
\hline & do $50 i=4,6$ & 33 \\
\hline 50 & $f(1, i)=0.0$ & 34 \\
\hline & $f(1,9)=$ seered $(9)$ & 35 \\
\hline & $f(1,8)=\operatorname{amin} 1(\operatorname{ttrans} *(a(1)-f(1,9))$, seered $(8))$ & 36 \\
\hline & $f(1,7)=\operatorname{amin} 1(\operatorname{ttrans} *(a(1)-f(1,9)-f(1,8))$, seered $(7))$ & 37 \\
\hline & $f(1,5)=\operatorname{amin} 1(\operatorname{ttrans} *(a(1)-f(1,9)-f(1,8)-f(1,7))$, seered $(5))$ & 38 \\
\hline & do $60 i=7,9$ & 39 \\
\hline 60 & $f(2, i)=0.0$ & 40 \\
\hline & $f(2,5)=0.0$ & 41 \\
\hline & $f(2,4)=\operatorname{transm} * a(2) * a(4) /(a(4)+a(6))$ & 42 \\
\hline & $f(2,6)=\operatorname{transm} * a(2) * a(6) /(a(4)+a(6))$ & 43 \\
\hline C & at this point all the viewfactors except those involving a(3), the & 44 \\
\hline c & distributed area, exist. one additional problem is that the & 45 \\
\hline c & interchange between enclosure areas no longer conserves energy & 46 \\
\hline c & because viewing area has been added to the low number surfaces. & 47 \\
\hline c & the strategy will be to "snitch" area from the lower right $5 \times 5$ & 48 \\
\hline c & matrix to account for the attenuation. some of this will be & 49 \\
\hline & relegated to interchange between $a(1 \ldots 2)$ and $a(4, .9)$. the rest & 50 \\
\hline
\end{tabular}


c will be sucked up by the term $f(i, 3)$. E 51

do $70 i=4,9 \quad 5 \quad 52$

do $80 j=4,9 \quad 5 \quad 53$

c write $(2, *) \cdot 1, i, j^{\prime}, j, j, f(i, j) \cdot, f(i, j)$, ' transm ',transm 54

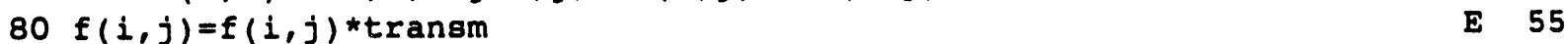

do $81 j=1,2 \quad 56$

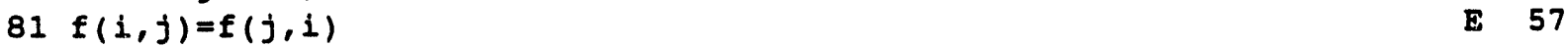

70 continue $r$ E

do $90 j=1,2 \quad 5 \quad 59$

$f(j, 3)=a(j) \quad$ E 60

do $100 i=1,9 \quad$ E 61

if (i.ne.3) $f(j, 3)=f(j, 3)-f(j, i) \quad$ E 62

100 continue $\quad$ E 63

90 continue $\quad$ E 64

\begin{tabular}{lr} 
C restore reciprocity & $\mathbf{E}$ \\
\hline
\end{tabular}

do $110 i=1,3 \quad$ E 66

do $110 j=3,9 \quad$ E 67

$\begin{array}{lr}110 f(j, i)=f(i, j) & \text { E }\end{array}$

do $120 j=4,9 \quad$ E 69

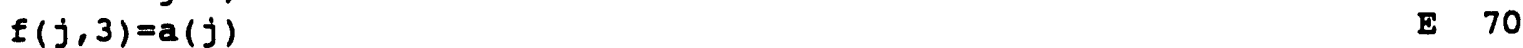

do $120 i=1,9 \quad$ E 71

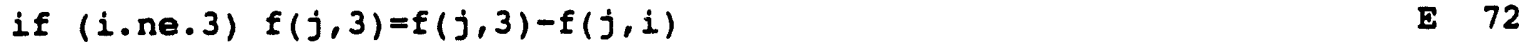

120 continue

do $140 j=4,9$

$140 f(3, j)=f(j, 3)$

$f(3,3)=a(3)$

do $150 i=1,9$

if (i.ne.3) $f(3,3)=f(3,3)-f(3, i) \quad$ E 78

150 continue $\quad$ E 79

c at this writing, 8/14/91, this routine seems to work acceptably $\mathrm{E} 80$

c for all reasonable inputs. two defects that are probably not $\mathrm{E}$

c worth eliminating are recognized. first, viewing area connecting $\mathrm{E} 82$

C a[1] and a[2] to surfaces of the sst interior a[4..8) are snitched $\mathbf{E} 83$

c from the views of a (3) to these surfaces. 84

$c$ if the "transmitance=1.0" then the views between $a(3)$ and these $\mathrm{E} 85$

c surfaces are zero so that this snitching results in $f(i, 3) \quad \mathbf{E}$

c ( $i$ in $4 . .8$ ) being negative since $f(i, 3)$ is calculated $\quad$ E 87

c from conservation. $\quad$ E

\begin{tabular}{lr} 
do $121 i=1,9$ & E \\
\hline
\end{tabular}

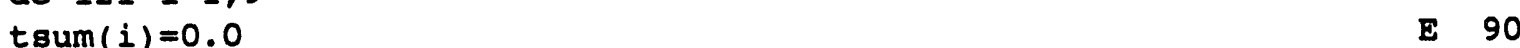

do $122 j=1,9 \quad$ E 91

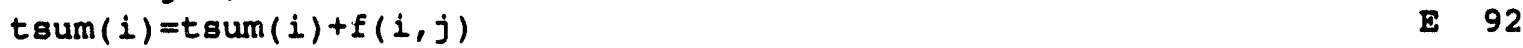

122 continue $r$

121 continue $r \begin{array}{ll}\text { E } & 94\end{array}$

$\begin{array}{lr}\text { return } & \text { E } 95\end{array}$

$\begin{array}{lr}\text { end } & \text { E } 96\end{array}$

subroutine $\operatorname{swap}(a, b) \quad F \quad 1$

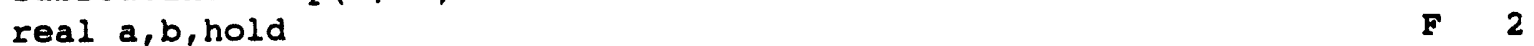

hold $=a \quad r .3$

$a=b \quad$ F 4

$\mathrm{b}=$ hold $\quad$ F 5

\begin{tabular}{lr} 
return & F \\
\hline end & 6
\end{tabular}

\begin{tabular}{lr} 
end & F \\
\hline
\end{tabular}

subroutine gaussj(b,y,coef,ncol,ierr) $\quad$ G 1 


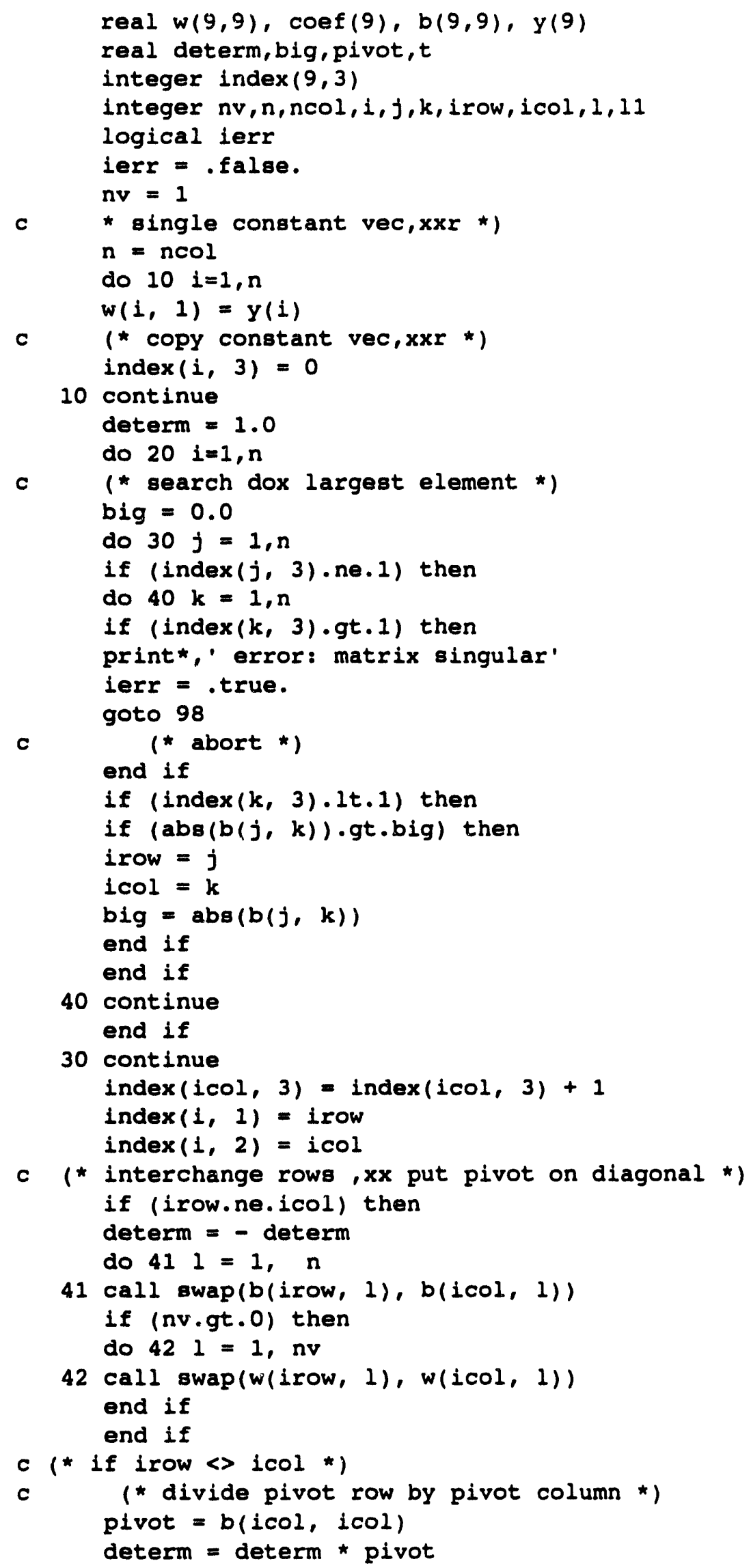

G 2

G 3

G 4

G 5

G 6

G 7

G 8

G 9

10

11

12

13

14

15

16

17

18

G 19

20

21

22

23

G 24

G 25

G 26

27

28

29

G 30

G 31

G 32

G 33

34

G 35

G 36

G 37

G 38

G 39

G 40

G 41

G 42

G 43

G 44

G 45

G 46

G 47

G 48

G 49

G 50

G 51

G 52

G 53

G 54

G 55 


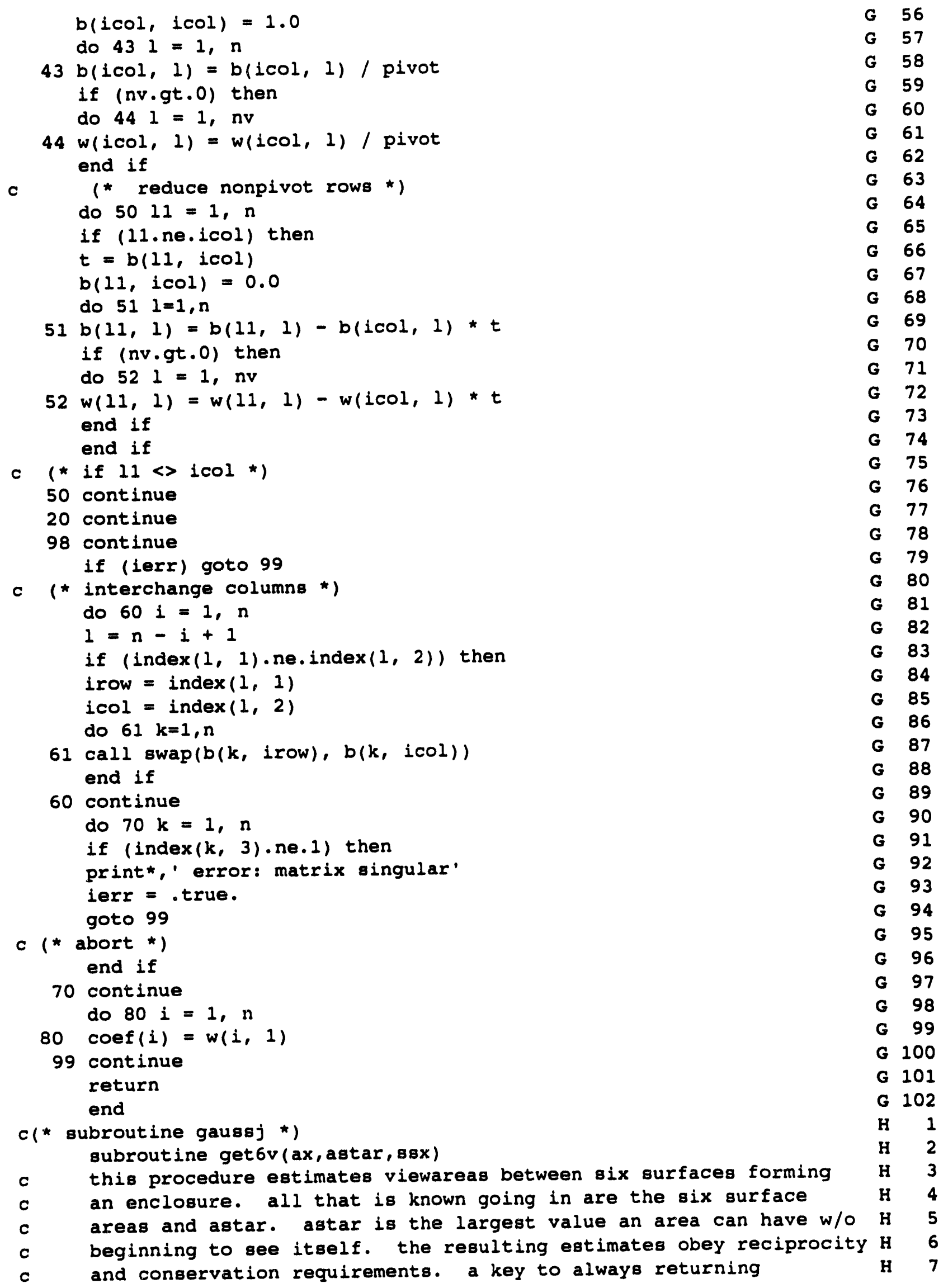


C plausible viewareas is that the area array is sorted and the 8

c smallest areas are treated first. Also, $f(5,6)=f(6,5)=0.0$. $\quad 9$

real $88(9,9), \operatorname{six}(9,9), \operatorname{ax}(9), \operatorname{axx}(9) \quad$ H 10

real awhole, seeme, astar, seeyou, sssum $\quad$ H 11

integer iloc( $(9), i, j$

do $10 i=1,6$

c write $(2, *)$ 'in loop', i

iloc $(i)=i$

$\operatorname{axx}(i)=\operatorname{ax}(i)$

10 continue

c write $\left.(2,)^{*}\right)$ just before call eorta'

call sorta(110c, axx, 6 )

c write $(2,)^{\prime}$ ' just after call sorta'

awhole $=0.0$

do $201=1,6$

20 awhole=awhole $+\operatorname{axx}(1)$

do $30 i=1,6$

do $40 j=i, 5$

if (i.eq.j) then

$\operatorname{si}(i, j)=$ geeme (axx (i), astar, awhole)

else

$\operatorname{ss}(i, j)=\operatorname{seeyou}(\operatorname{axx}(i), \operatorname{astar}, \operatorname{axx}(j)$, awhole)

C

surfaces 4 and 5 are both floor, they can't see each other

if $((i \operatorname{loc}(i)+i \operatorname{loc}(j))$.eq. 3$) \mathrm{sg}(i, j)=0.0$

end if

40 continue

if (i.gt.1) then

c

invoke reciprocity

do $50 j=1,1-1$

$50 \mathrm{8s}(i, j)=88(j, i)$

end if

Bgsum $=0.0$

use conservation to compute each surface's view to the largest surface

do $60 j=1,5$

60 sgsum $=8$ sgum $+88(i, j)$

$88(i, 6)=\operatorname{axx}(i)-888 u m$

30 continue

c

place results in the array in the "unsorted" order

do $70 i=1,6$

do $70 j=1,6$

H 12

H 13

H 14

H 15

H 16

H 17

H 18

H 19

H 20

H 21

H 22

H 23

H 24

H 25

H 26

H 27

H 28

H 29

H 30

H 31

H 32

H 33

H 34

H 35

H 36

H 37

H 38

H 39

H 40

H 41

H 42

H 43

H 44

H 45

H 46

H 47

H 48

$70 \operatorname{sgx}(i \operatorname{loc}(i), i \operatorname{loc}(j))=88(i, j)$

c write $(2,)^{\prime} 88 x$ just before leaving get $6 v^{\prime}$

c write $\left(2,{ }^{*}\right) 88 x$

return

end

real function seeme(al,astar, awhole)

real al, astar, awhole

if (a1.1t.astar) then

seeme $=0.0$

else

seeme=al *astar / (awhole-astar)

end if

return 


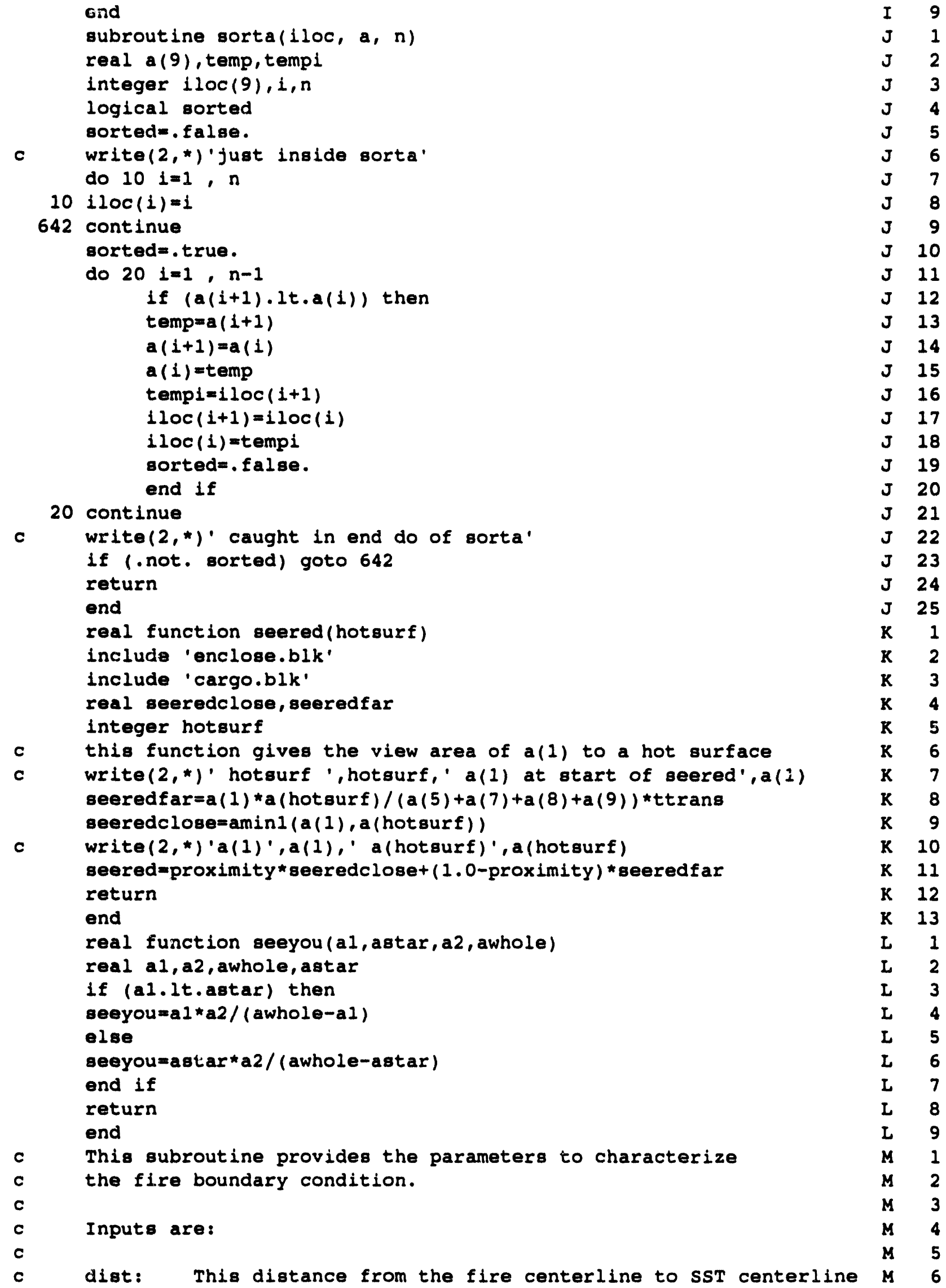




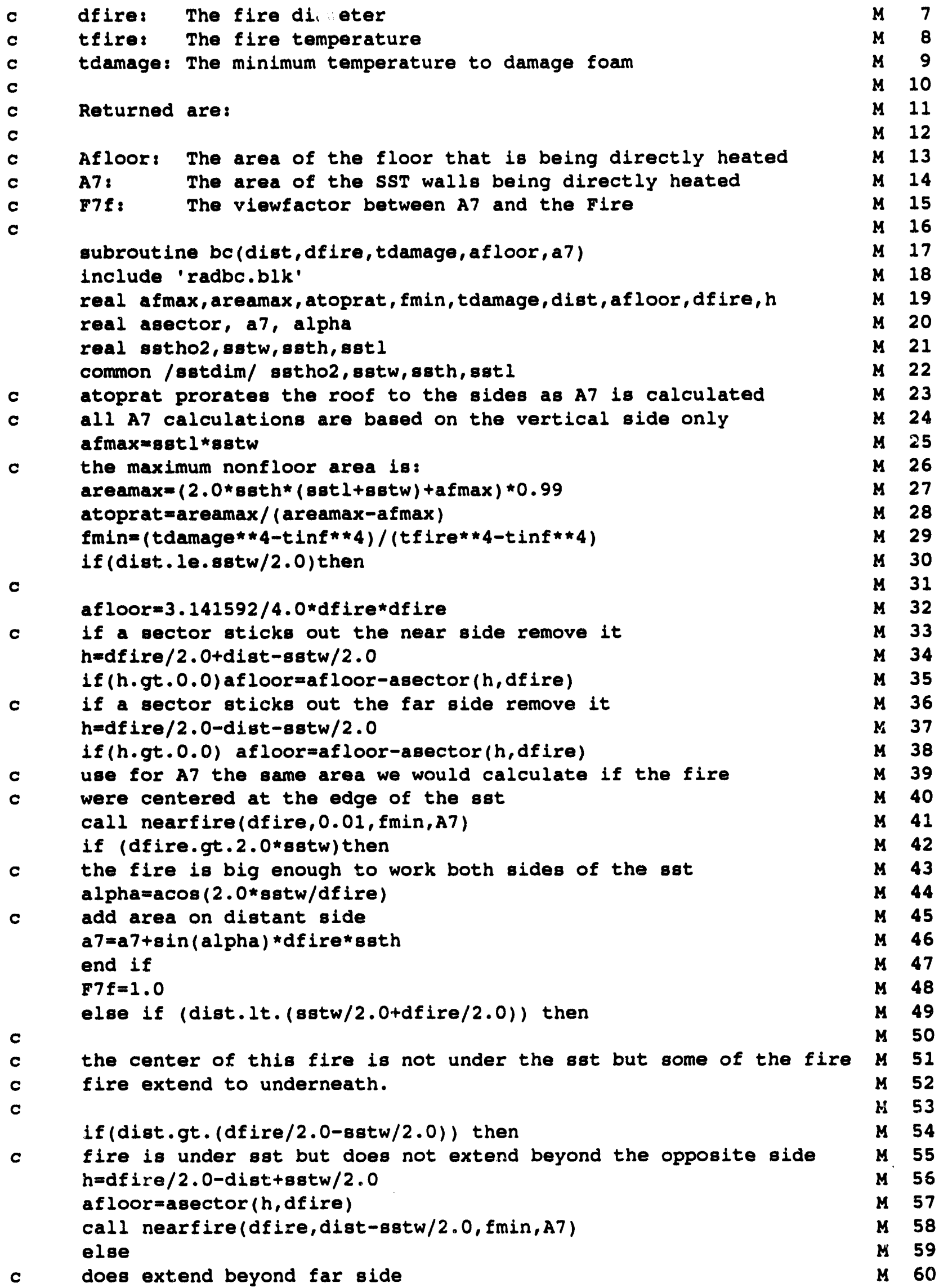



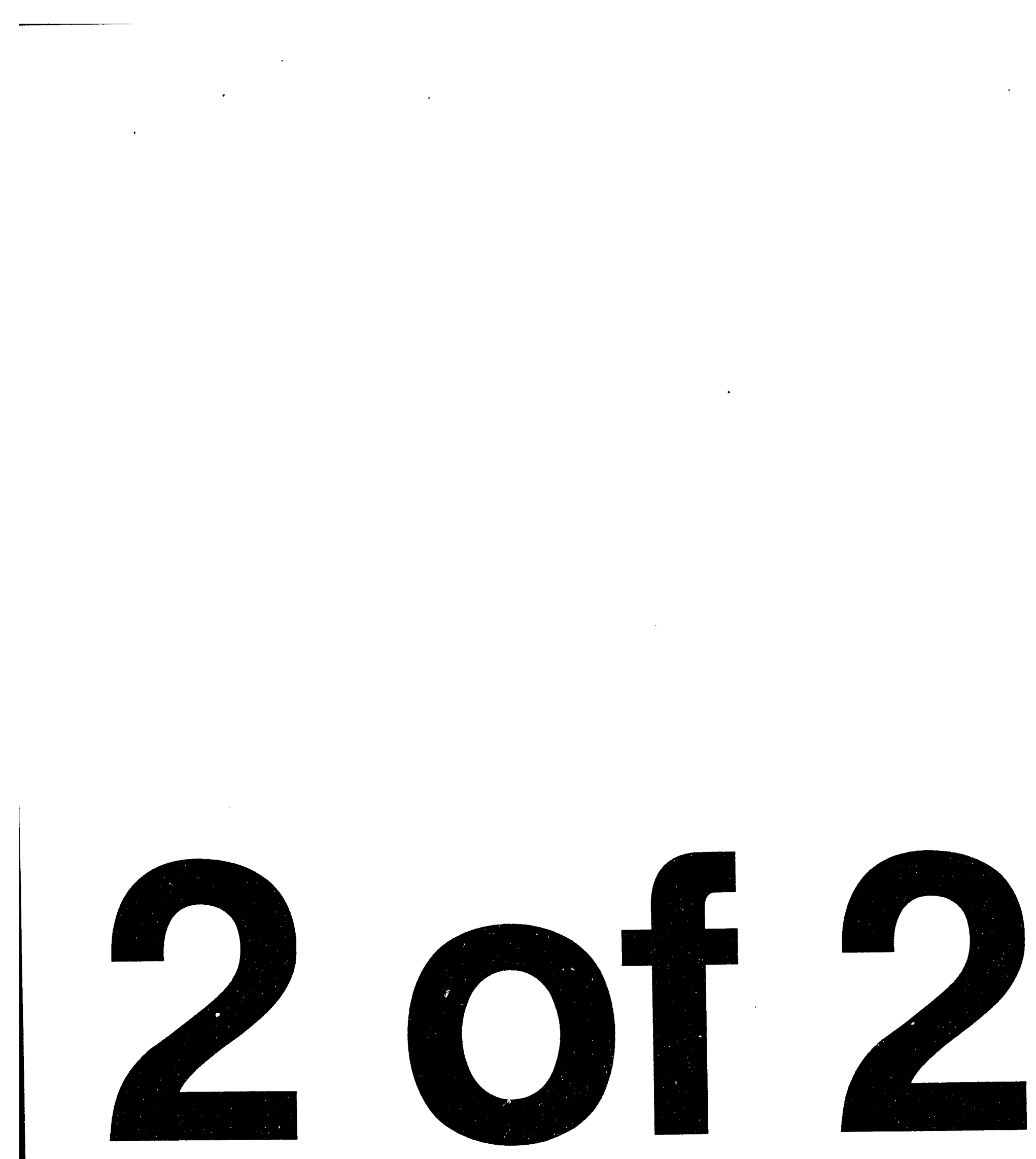
$h=d f i r e / 2.0-d i s t+s s t w / 2.0$

M 61

afloor=asector ( $h, d f$ ire)

$\mathrm{h}=\mathrm{df}$ ire $/ 2.0-\mathrm{dist}-8 \mathrm{st} \mathrm{w} / 2.0$

M 62

afloor=afloor-asector ( $h$, df ire)

M 63

alpha $=\operatorname{acos}(1.0-2.0 * \mathrm{~h} / \mathrm{df}$ ire $)$

C

get nearside a7 by calling near fire

call nearfire(dfire, dist-sstw/2.0, fmin, A7)

M 64

M 65

add area on distant side

a $7=a 7+\sin ($ alpha $)$ *dfire*ssth

$f 7 f=1.0$

end if

else

c fire is completely removed from sst

afloor $=0.0$

call farfire(dfire, dist-sstw/2.0,fmin, a 7)

M 66

M 67

M 68

M 69

M 70

$M 71$

M 72

M 73

end if

a 7=a 7*atoprat

if (a7.gt.areamax) a $7=a r e a m a x$

if (afloor.gt.afmax) afloor=afmax

return

end

subroutine nearfire(diafire,dist, fmin,a7)

M 74

M 75

M 76

M 77

$M 78$

M 79

M 80

M 81

C

c This routine is called if the fire does touches the SST $\quad$ N 3

N 1

N 2

include 'radbc.blk'

real sstho2, sstw, ssth, sst 1

common /sstdim/ sstho2, sstw, ssth, sst l

real hfire,diafire, dhf, dist, a 7, ecum, dwsst, $x$, flocal, v2fire

real fmin, hw

integer $i$

c

calculate the half width of the wall portion touching the fire $f 7 f=1.0$

hfire $=3.0 *$ diafire

dhf $=\operatorname{sqrt}($ diafire $* \star 2 / 4.0-d i s t \star \star 2)$

c

if (dhf.gt.sstl/2.0)then

c the fire covers the side of the sat

a 7 $=88$ s 1 *gsth

return

end if

C

ecum $=d h f^{*}$ efire

dwsgt $=(8 s t l / 2.0-d h f) / 40.0$

$x=d h f$

N 4

N 5

N 6

N 7

N 8

N 9

N 10

N 11

N 12

N 13

N 14

N 15

N 16

N 17

N 18

N 19

N 20

N 21

N 22

N 23

N 24

c

if the following do loop goes to its upper limit the integration

N 25

is along the whole side of the sst. If the fire conditions are $N \quad 26$

such that fmin occurs somewhere along that length, the upper $\quad 27$

limit of integration stops there.

N 28

do $10 i=1,40$

N 29

$x=d w s s t+x$

N 30

N 31

flocal=v2fire(dist, $(i-1) \star$ dwsst+dhf, sstho2, diafire,hfire) $\quad 32$

ecum=dwsst* (einftflocal* (efire-einf $))+e c u m$

N 33 


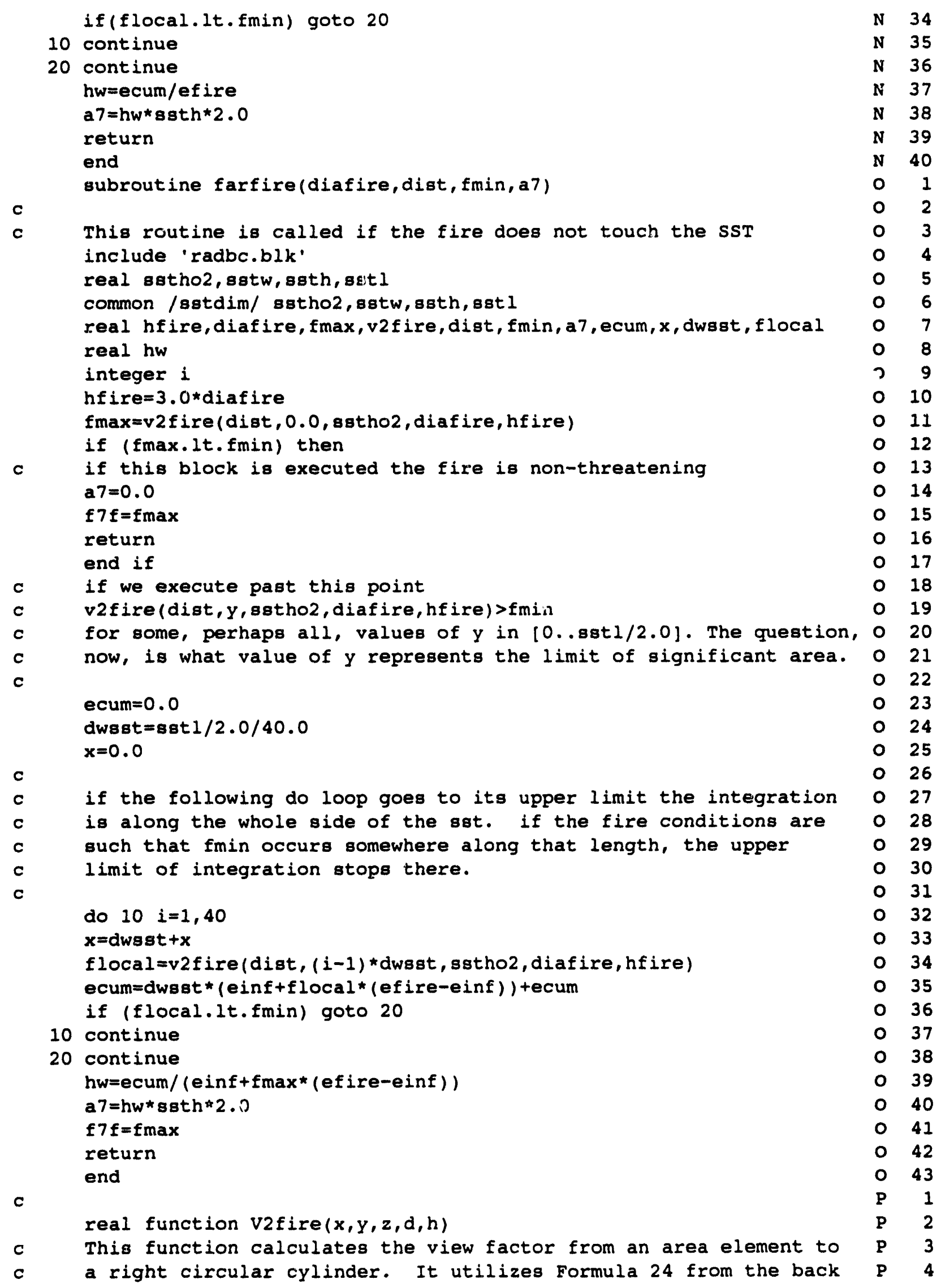




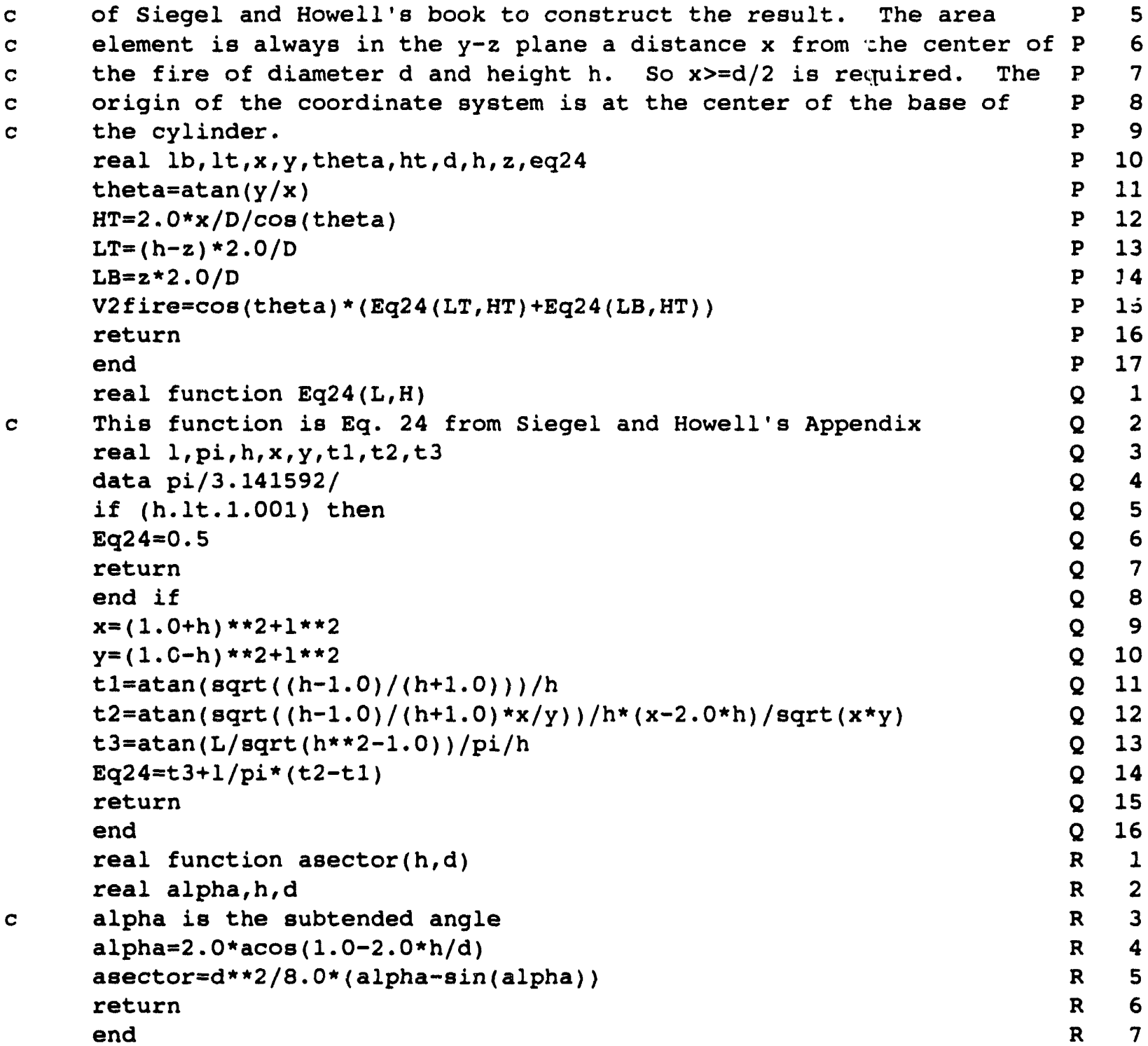




\begin{tabular}{|c|c|c|}
\hline \multicolumn{3}{|c|}{ Itreactw, treactf } \\
\hline & $2 / 305.0,305.0,20.18,12440.0,12440.0,0.052,0.052,585.0,585.0 /$ & 16 \\
\hline & data eps $/ 8 * 0.7,1.0 /$ & 17 \\
\hline 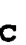 & 28400 is the armor's rho*cp*thickness product & 18 \\
\hline & data $\mathrm{rch} / 3 * 1.0,15000.0,27500.0,3 * 28400.0 /$ & 19 \\
\hline & data rchskinw, rchskinf $/ 2 * 2525.0 /$ & $\mathbf{x}$ \\
\hline C & the steady state resistance of virgia plywood is used & $\mathrm{x}$ \\
\hline & along with it's thermal capacitance, $1 / 2$ " thickness & $\mathrm{x}$ \\
\hline & data rchply, reply/12500.0,0.06/ & 23 \\
\hline & data epso 5 , epso 7, epso $8, \operatorname{tinf} / 3 \star 0.9,300.0 /$ & 24 \\
\hline & end & 25 \\
\hline
\end{tabular}


CARGO.BLK, declarations of variables associated with cargo parameters

real proximity, ck, cr,mcp, rcarg, acargo, cargoeps, transm, ttrans, split integer nstrip, ncs, model number, ncargo, ncrit common /cargo/proximity, ck (10), cr (10), mcp (10), rcarg(10), acargo, lcargoeps, transm, ttrans, split, nstrip, ncs, modelnumber, ncargo, ncrit

ENCLOSE.BLK, declarations of variables associated with enclosure parameters.

real a, arad, hh, eps, epsrad, rmcp3, rch, rchskinf, rchskinw, rchply, reply integer isurf, aid, qid, nsurfrad, skin7id,plyid, skin5id, char7id,

1 char 5 id

logical ahere

common /enclose/a (9), arad $(9), h h(9,9), \operatorname{eps}(9), \operatorname{epsrad}(9)$,

Irmcp 3, rch (8), rchskinf, rchskinw, rohply, reply, isurf (30), aid (30), 2qid(30), nsurfrad, skin7id, plyid, skin5id, char7id, char5id, ahere (9)

FOAM.BLK, declarations of variables associated with foam wall parameters

real lwall, lfloor, kvirg, heatregw, heatreqf, aawall

real aafloor, treactw, treact $f$, ereact $f$, ereactw

common /foam/lwall, lfloor, kvirg, heatreqw, heatreqf, aawall,

laafloor, treactw, treactf, ereactf, ereactw

MELT.BLK, declarations of variables associated with the melting of the outside shell of the cargo

real emelt, umelt, tmelt, tmelted, arat

logical melting, melted, cool, canmelt

common /melt/emelt, umelt, tmelt, tmelted, arat, melting, melted, cool, lcanmelt

RADBC.BLK, declarations of variables associated with representing the radiation boundary condition

real epso5,epso7, epso8,tfire,tfiref,tinf,efire, efiref, leinf, $f 5 f, f 7 f, f 8 f, f 9 f$, firerate, sigma, ehol: common / radbc/epso5, epso7, epso8, tfire, $t$ iref,tinf, efire, efiref, leinf, $f 5 f, f 7 f, f 8 f, f 9 f, f i r e r a t e, s i g m a$, ehole 


\title{
Distribution
}

\author{
6 U.S. Department of Energy \\ Attn.: $\quad$ E. G. Grewis, DP-20.1 \\ D. R. Olson, DP-21 \\ C. G. Halsted, DP-22 \\ J. D. Rodgers, DP-22 \\ J. R. Maisonet, DP-22 \\ T. J. Goodwin, DP-22 \\ 19901 Germantown Rd. \\ Germantown, MD 20874 \\ 6 \\ U.S. Department of Energy \\ Albuquerque Operations Office \\ Attn.: $\quad$ G. E. Runkle, HPD \\ G. V. Binns, NESD \\ R. O. Inlow, OSS \\ R. E. Sabre, TSD \\ R. H. Richey, TSD \\ D. B. Umshler, WPD \\ P.O. Box 5400 \\ Albuquerque, NM 87185-5400 \\ 2 \\ Los Alamos National Laboratory \\ Attn.: $\quad$ A. A. Guthrie, ESA-11, MS C931 \\ D. A. O'Brien, TSA-9, MS F684 \\ P.O. Box 1663 \\ Los Alamos, NM 87545 \\ Lawrence Livermore National Laboratory \\ Attn.: $\quad$ G. L. Dittman, L-125 \\ P.O. Box 808 \\ Livermore, CA 94550 \\ 1 \\ Logicon R\&D Associates \\ Attn: $\quad$ S. F. Woodford \\ 6940 S. Kings Highway, Suite 202 \\ Alexandria, VA 22310 \\ 1 \\ R. H. Odegaarden \\ 6048 E. Star Valley Street \\ Mesa, AZ 85215-9609
}




\section{(Distribution Continued)}

1

1

J. S. Phillips 925 E. Navajo

Farmington, NM 87401

J. T. Risse

28 Riverside Rd.

Peralta, NM 87042

$\begin{array}{ll}\text { MS0405 } & \text { D. D. Carlson, 6411 } \\ \text { MS0491 } & \text { R. E. Smith, 12333 } \\ \text { MS0492 } & \text { G. A. Sanders, 12332 } \\ \text { MS0492 } & \text { C. G. Shirley, 12332 } \\ \text { MS0560 } & \text { D. F. McVey, 5408 } \\ \text { MS0631 } & \text { R. W. Schwoebel, 12300 } \\ \text { MS0763 } & \text { W. F. Hartman, 9614 } \\ \text { MS0763 } & \text { D. B. Clauss, 9614 } \\ \text { MS0763 } & \text { R. D. Klett, 9614 } \\ \text { MS0763 } & \text { M. E. Larsen, } 9614 \\ \text { MS0763 } & \text { R. K. Wilson, 9614 } \\ \text { MS0766 } & \text { J. R. Kelsey, } 9600 \\ \text { MS0767 } & \text { E. R. Hoc 2r, 9603 } \\ \text { MS0767 } & \text { S. C. Roehrig, } 9604 \\ \text { MS0790 } & \text { H. J. Abeyta, } 9612 \\ \text { MS0790 } & \text { B. D. Boughton, 9612 } \\ \text { MS0790 } & \text { J. J. Roesch, 9613 } \\ \text { MS0790 } & \text { R. G. Baca, } 9613 \\ \text { MS0835 } & \text { J. L. Moya, 1513 } \\ \text { MS9018 } & \text { Central Technical Files, 8523-2 } \\ \text { MS0899 } & \text { Technical Library, 13414 } \\ \text { MS0619 } & \text { Technical Publications, 13416 } \\ \text { MS1119 } & \text { Document Processing for DOE/OSTI, 7613-2 }\end{array}$



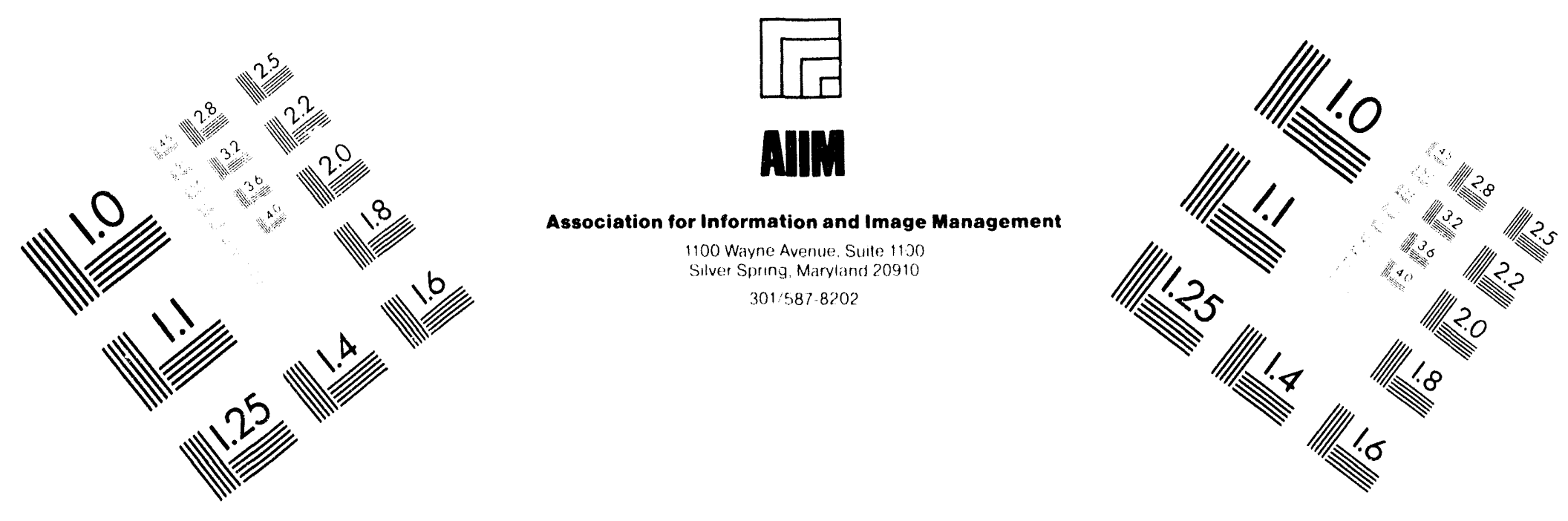

\section{Centimeter}

1
$\mid$

Inches
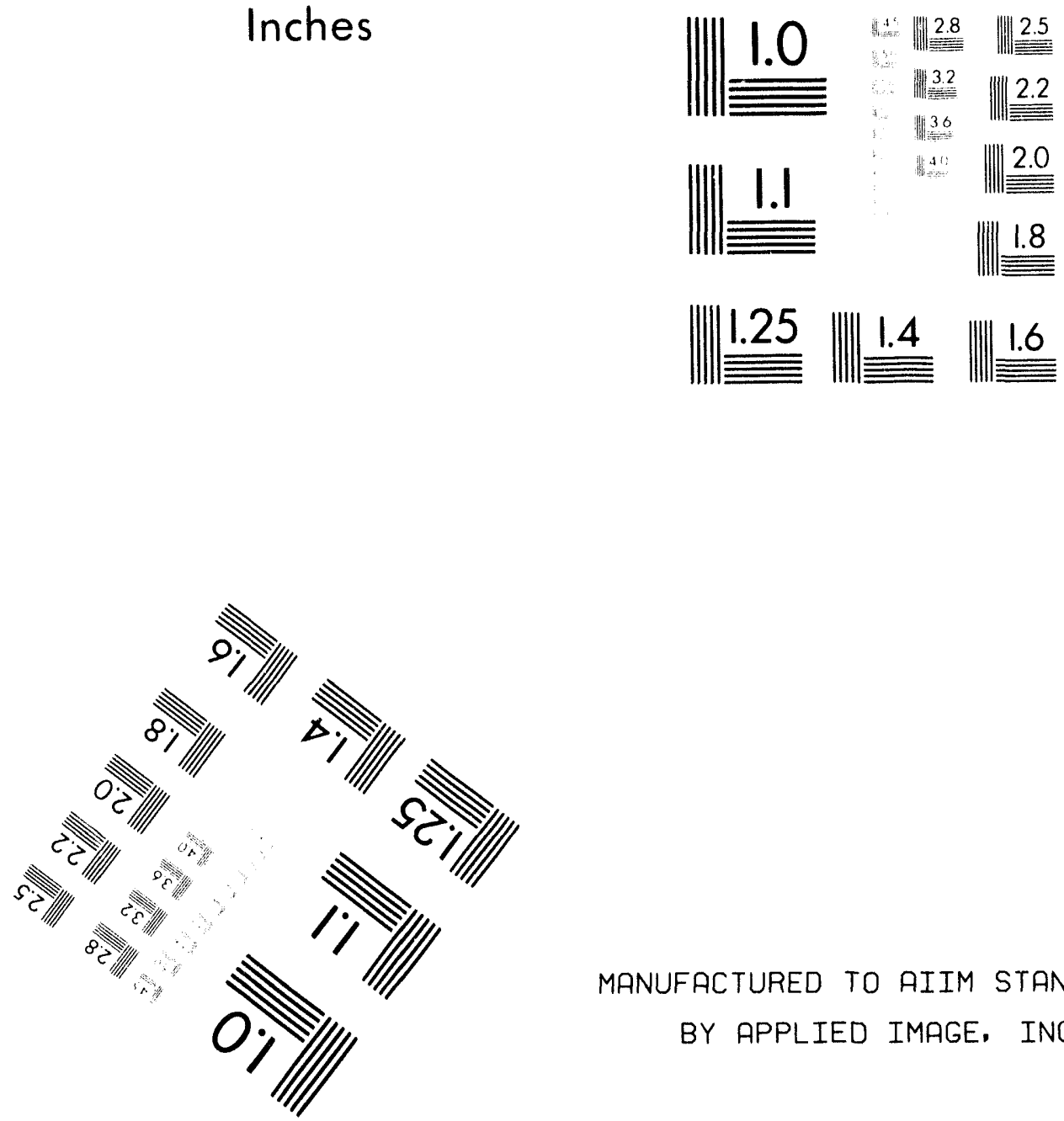

MANUFACTURED TO AIIM STANDARDS

BY APPLIED IMAGE, INC.

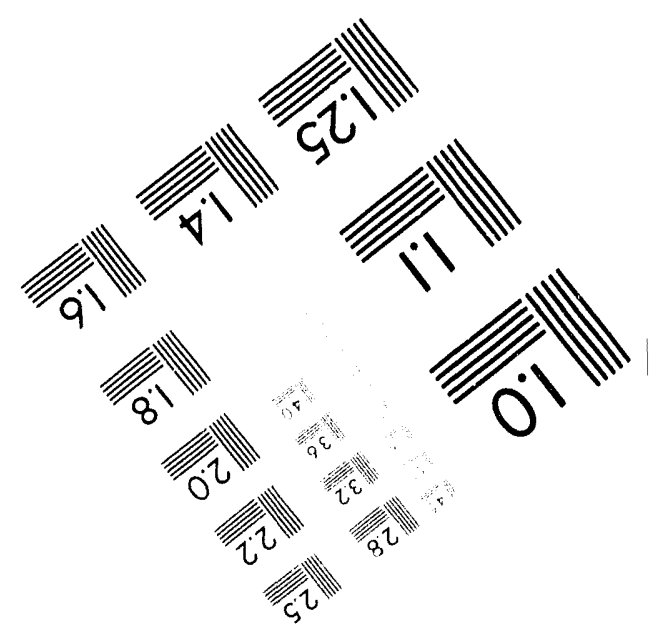



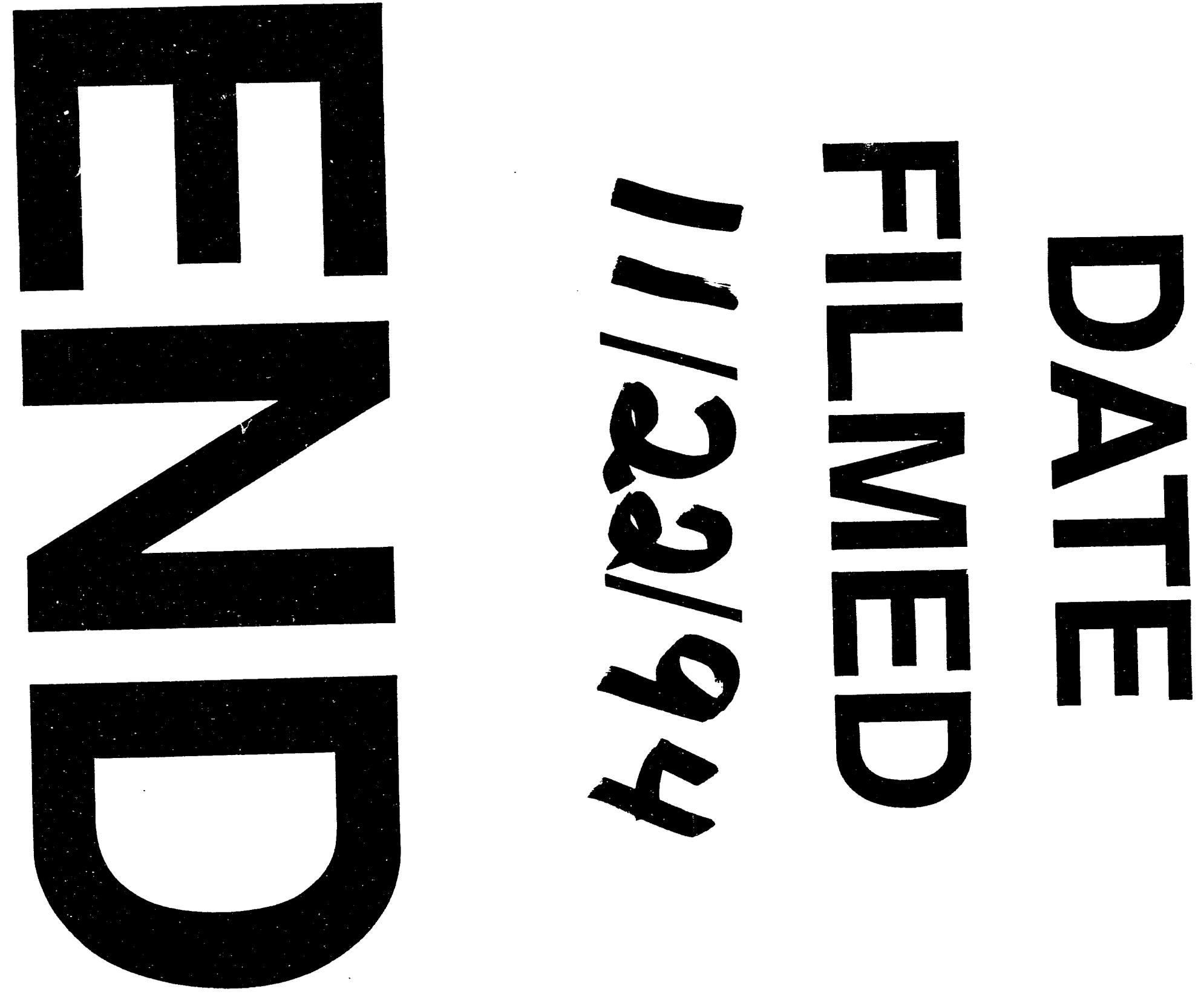